\title{
Epidemiological and statistical basis for detection and prediction of influenza epidemics
}

\author{
Armin Spreco
}

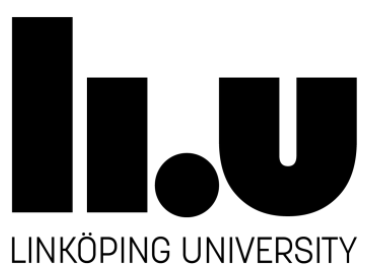

Faculty of Medicine and Health Sciences

Division of Community Medicine

Department of Medical and Health Sciences

Linköping University, Sweden 
Epidemiological and statistical basis for detection and prediction of influenza epidemics

(CArmin Spreco, 2017

Published articles have been reprinted with the permission of the copyright holders.

Printed in Sweden by LiU-Tryck, Linköping, Sweden, 2017

ISBN: 978-91-7685-569-0

ISSN: 0345-0082 


\section{"Read in the name of your Lord who created, Created man from an embryo; \\ Read, for your Lord is most beneficent, Who taught by the pen, \\ Taught man what he did not know."}

- The Holy Qur'an 96:1-5 (Translation of the first five verses revealed by God Almighty to Prophet Mohammad, peace and blessings be upon him). 



\section{CONTENTS}

ABSTRACT.

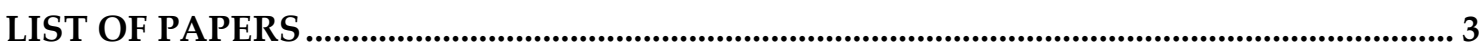

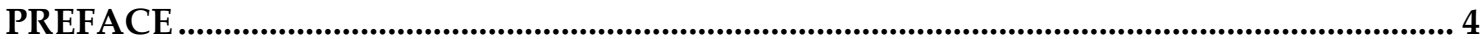

INTRODUCTION

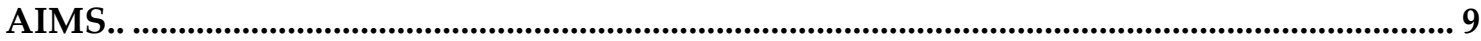

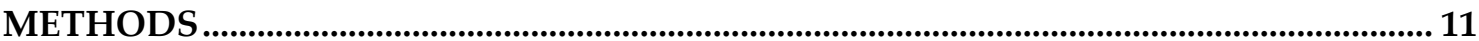

Preparatory phase: examination of eHealth data sources ...................................................................12

Performance of eHealth data sources in local influenza surveillance........................................................12

Study design

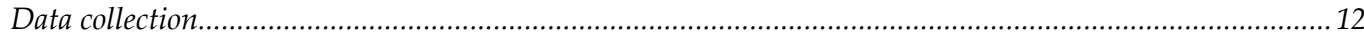

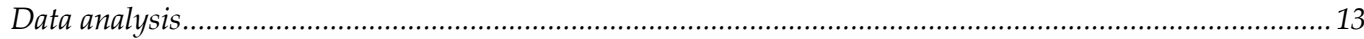

Preparatory phase: review and evaluation of existing detection and prediction algorithms ....14

Meta-narrative review and comparative trial...……………………………………………………......14

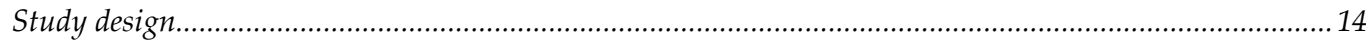

Meta-narrative review: data collection and analysis ......................................................................... 16

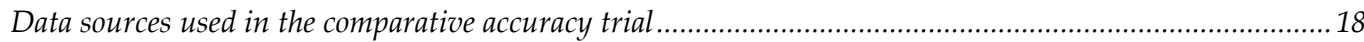

Evaluation procedure in the comparative accuracy trial .......................................................................... 19

Algorithms evaluated and excluded in the comparative accuracy trial ......................................................20

Development phase: design and evaluation of the nowcasting method ..........................................21

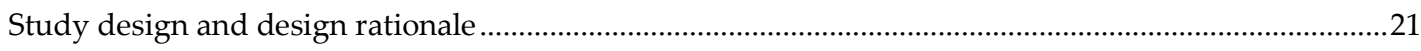

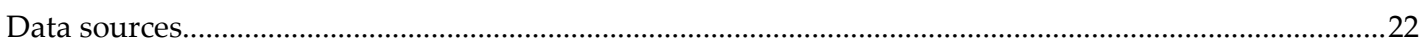

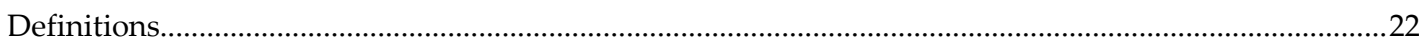

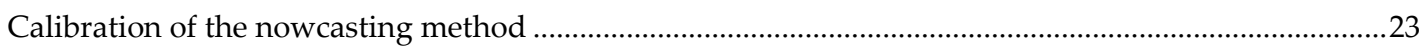

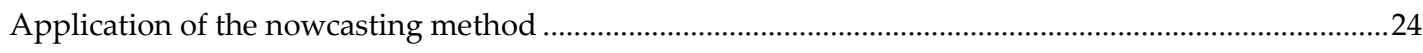

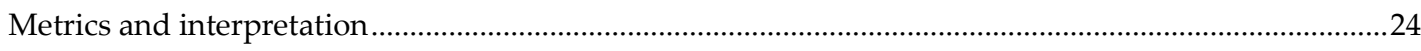

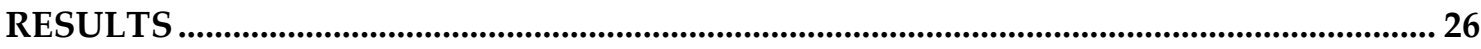

Preparatory phase: examination of eHealth data sources ................................................................26

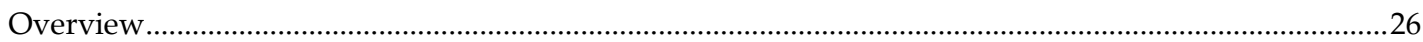

Correlations between local media coverage, influenza-diagnosis cases, and eHealth data .....................27

Correlations between GFT and influenza-diagnosis data ......................................................................28

Correlations between telenursing call data and influenza-diagnosis data ................................................29

Correlations between log data from the county council website and influenza-diagnosis data ...............30 
Correlations between GFT data, telenursing data, and log data from the county council website .30

Preparatory phase: review and evaluation of existing detection and prediction algorithms ....32

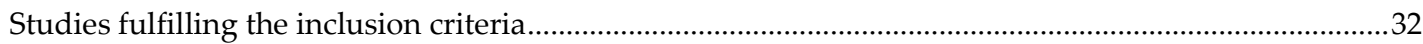

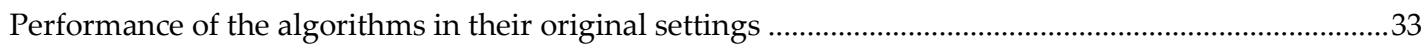

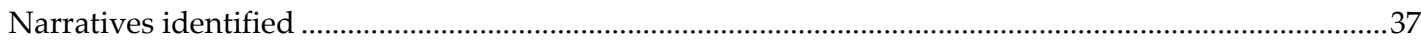

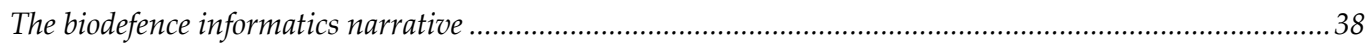

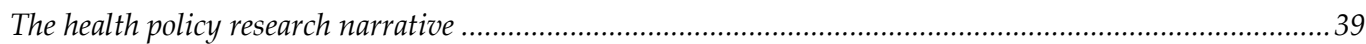

Algorithms considered for the comparative accuracy trial ......................................................................40

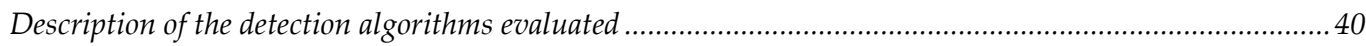

Description of the prediction algorithms evaluated ................................................................................... 42

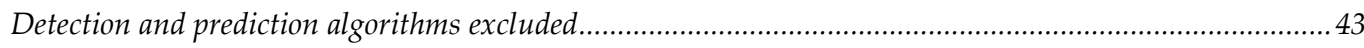

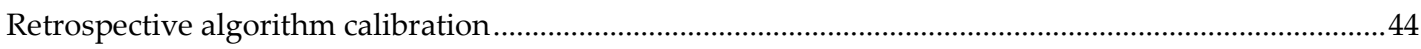

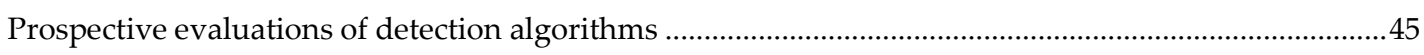

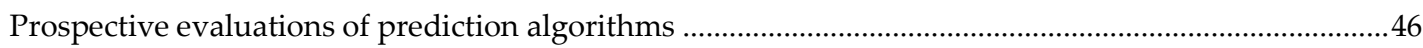

Development phase: design and evaluation of the nowcasting method ......................................47

Method design overview …………..................................................................................................................... 47

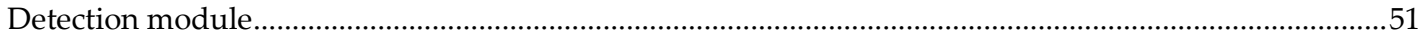

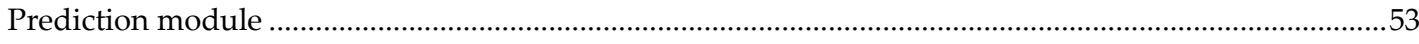

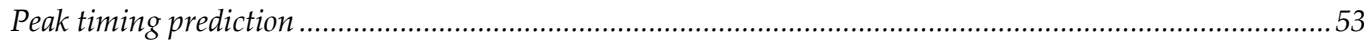

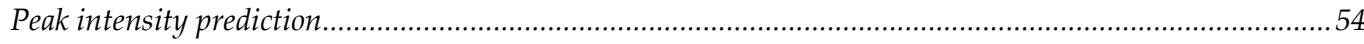

Retrospective performance of the nowcasting method ..................................................................................55

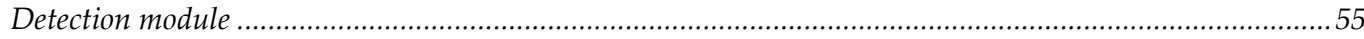

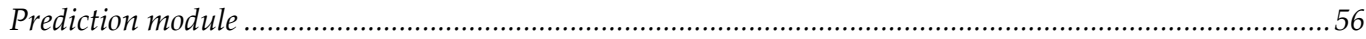

Prospective evaluation of the nowcasting method .......................................................................................57

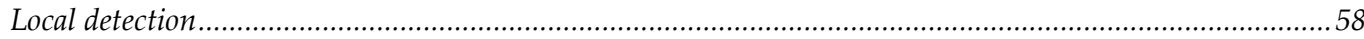

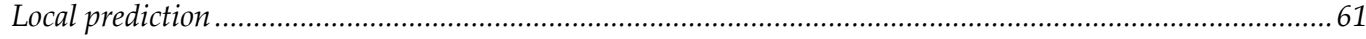

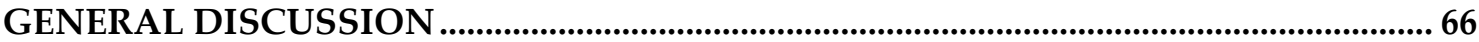

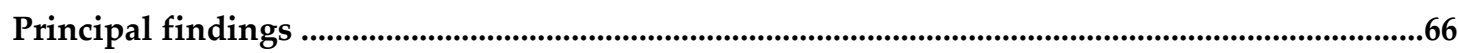

Preparatory phase: examination of eHealth data sources...........................................................................66

Preparatory phase: review and evaluation of existing detection and prediction algorithms....................67

Development phase: design and evaluation of the nowcasting method ...................................................68

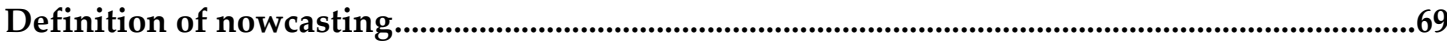

Prospective evaluations of detection and prediction algorithms: the way to go ..........................69

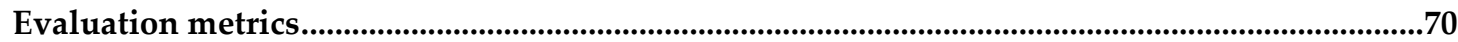




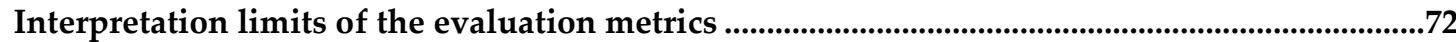

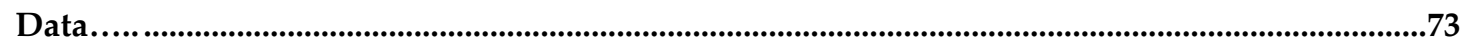

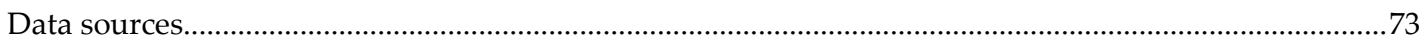

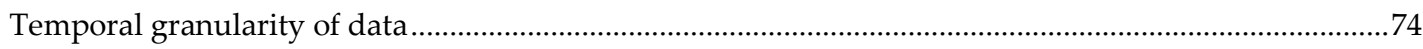

The nowcasting method design: comparison with previous work ....................................................75

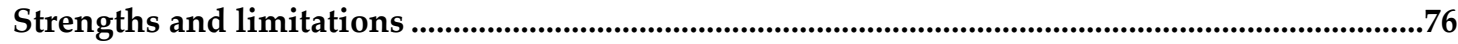

Preparatory phase

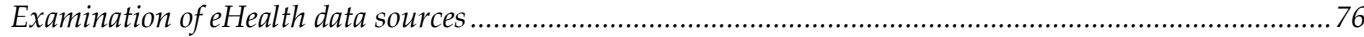

Meta-narrative review and comparative accuracy trial .............................................................................. 76

Development phase: design and evaluation of the nowcasting method .....................................................78

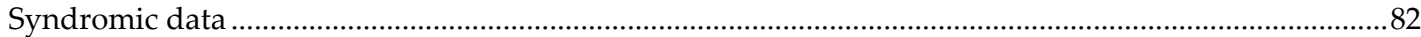

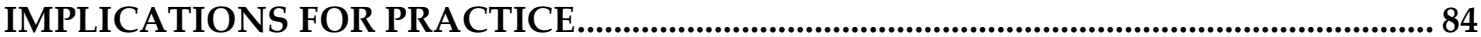

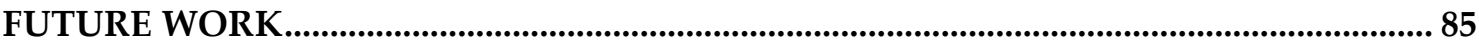

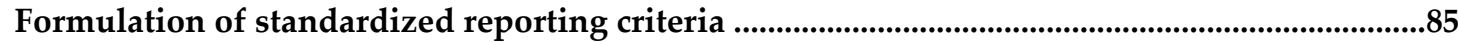

Further development and evaluation of the nowcasting method ................................................85

Application of the nowcasting method on other syndromic data .................................................86

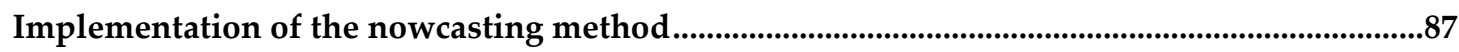

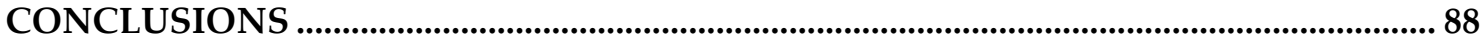

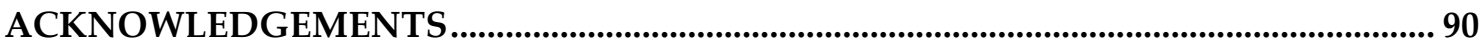

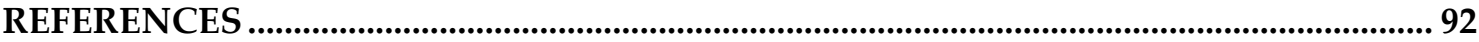





\section{ABSTRACT}

A large number of emerging infectious diseases (including influenza epidemics) has been identified during the last century. The emergence and re-emergence of infectious diseases have a negative impact on global health. Influenza epidemics alone cause between 3 and 5 million cases of severe illness annually, and between 250,000 and 500,000 deaths. In addition to the human suffering, influenza epidemics also impose heavy demands on the health care system. For example, hospitals and intensive care units have limited excess capacity during infectious diseases epidemics. Therefore, it is important that increased influenza activity is noticed early at local levels to allow time to adjust primary care and hospital resources that are already under pressure. Algorithms for the detection and prediction of influenza epidemics are essential components to achieve this.

Although a large number of studies have reported algorithms for detection or prediction of influenza epidemics, outputs that fulfil standard criteria for operational readiness are seldom produced. Furthermore, in the light of the rapidly growing availability of "Big Data" from both diagnostic and prediagnostic (syndromic) data sources in health care and public health settings, a new generation of epidemiologic and statistical methods, using several data sources, is desired for reliable analyses and modeling.

The rationale for this thesis was to inform the planning of local response measures and adjustments to health care capacity during influenza epidemics. The overall aim was to develop a method for detection and prediction of influenza epidemics. Before developing the method, three preparatory studies were performed. In the first of these studies, the associations (in terms of correlation) between diagnostic and pre-diagnostic data sources were examined, with the aim of investigating the potential of these sources for use in influenza surveillance systems. In the second study, a literature study of detection and prediction algorithms used in the field of influenza surveillance was performed. In the third study, the algorithms found in the previous study were compared in a prospective evaluation study. In the fourth study, a method for nowcasting of influenza activity was developed using electronically available data for real-time surveillance in local settings followed by retrospective application on the same data. This method includes three functions: detection of the start of the epidemic at the local level and predictions of the peak timing and the peak intensity. In the 
fifth and final study, the nowcasting method was evaluated by prospective application on authentic data from Östergötland County, Sweden.

In the first study, correlations with large effect sizes between diagnostic and pre-diagnostic data were found, indicating that pre-diagnostic data sources have potential for use in influenza surveillance systems. However, it was concluded that further longitudinal research incorporating prospective evaluations is required before these sources can be used for this purpose. In the second study, a meta-narrative review approach was used in which two narratives for reporting prospective evaluation of influenza detection and prediction algorithms were identified: the biodefence informatics narrative and the health policy research narrative. As a result of the promising performances of one detection algorithm and one prediction algorithm in the third study, it was concluded that both further evaluation research and research on methods for nowcasting of influenza activity were warranted. In the fourth study, the performance of the nowcasting method was promising when applied on retrospective data but it was concluded that thorough prospective evaluations are necessary before recommending the method for broader use. In the fifth study, the performance of the nowcasting method was promising when prospectively applied on authentic data, implying that the method has potential for routine use. In future studies, the validity of the nowcasting method must be investigated by application and further evaluation in multiple local settings, including large urbanizations. 


\section{LIST OF PAPERS}

The thesis is based on the following papers:

I. Timpka T, Spreco A, Dahlström Ö, Eriksson O, Gursky E, Ekberg J, Blomqvist E, Strömgren M, Karlsson D, Eriksson H, Nyce J, Hinkula $\mathrm{J}$, Holm E. Performance of eHealth data sources in local influenza surveillance: a 5-year open cohort study. J Med Internet Res 2014;16(4):e116.

II. Spreco A, Timpka T. Algorithms for detecting and predicting influenza outbreaks: metanarrative review of prospective evaluations. BMJ Open 2016;6(5):e010683.

III. Spreco A, Eriksson O, Dahlström Ö, Timpka T. Influenza detection and prediction algorithms: comparative accuracy trial in Östergötland County, Sweden, 2008-2012. Submitted.

IV. Spreco A, Eriksson O, Dahlström Ö, Cowling BJ, Timpka T. Design of algorithms for integrated detection and prediction of influenza activity for real-time surveillance. Submitted.

V. Spreco A, Eriksson O, Dahlström Ö, Cowling BJ, Timpka T. Integrated nowcasting (detection and prediction) of influenza activity for real-time surveillance in local settings: prospective evaluation in Östergötland County, Sweden, 2009-2014. Submitted.

These papers are printed with permission from the publishers.

The project was approved by the Regional Research Ethics Board in Linköping (dnr. 2012/104-31). 


\section{PREFACE}

This thesis contributes to a central part of the public health tradition in that statistical and mathematical models are developed for implementation in health service practice with the purpose of making the service more effective with regard to patient care and societal costs. I came to the field of public health from statistics and found the concept of not only attempting to build these kinds of models, but also understanding the underlying theoretical mechanisms of public health phenomena and applying developed models in practical public health settings stimulating and refreshing.

Research in the public health field proved to be more complex than I initially thought. One observation I made while performing the research for this thesis is that it is necessary to involve researchers from various disciplines, such as epidemiology, statistics, mathematics, behavioral science, etc., when building models to be used in practice. Thanks to mentors in academia, public health, as well as statistics and behavioral science, I believe that the research in this thesis incorporates aspects of all these disciplines to some extent.

I am grateful for being given the opportunity to do research and to cooperate with high-class experts in this field. I hope that the research in my thesis will help improve the effectiveness of infectious disease surveillance and I look forward to contributing in the field of public health for many years to come.

Armin Spreco 


\section{INTRODUCTION}

A large number of infectious diseases (including influenza epidemics) have emerged and been identified during the last century. In a relatively recent report, 335 infectious diseases were identified between 1940 and 2004, including HIV, malaria and severe acute respiratory syndrome (Jones et al. 2008). During the last decade, other infectious diseases have emerged and reemerged, such as aH1N1 in 2009 and Ebola virus disease in 2014. The emergence and re-emergence of infectious diseases have had a highly significant impact not only on global health (Morens et al. 2004) but also on economies (Binder et al. 1999). Influenza epidemics alone cause 3-5 million cases of severe illness, and about 250,000-500,000 deaths, especially among pregnant women, elderly people and children aged 6-59 months (WHO 2016). In addition to human suffering, influenza epidemics also impose huge costs on society as a result of, e.g. high levels of absenteeism and heavy demands on the health care system (Szucs 1999, Molinari et al. 2007). For example, in a recent study in England, it was found that for each child diagnosed with influenza, the mean work absence for caregivers was approximately 4 days (Thorrington et al. 2017). Also, hospitals and intensive care units have limited excess capacity during emerging epidemics of infectious diseases (Donker et al. 2011). In Sweden, the hospital bed capacity is habitually overextended with on average 103 patients occupying 100 regular hospital bed units (SKL 2016).

As a result of recent technical developments in the infrastructure of public health information, today it is realistic to collect, structure, and statistically analyze infectious disease data in close to real time and in local public health contexts (Timpka et al. 2011). Early knowledge of influenza epidemics in the community allows local epidemic alerts in primary care and hospital settings before the publication of regional data and could accelerate the implementation of preventive transmission-based precautions both within the local health care services and the community (Gerbier-Colomban et al. 2014). Therefore, it is important that an increase in influenza activity is noticed early at the local level to allow time for primary care and hospital resources already under pressure to meet the demand in the community (especially hospitalizations requiring intensive care). Numerous attempts have been made to develop epidemiological methods for these purposes (see Singh et al. 2010, Dórea et al. 2013a, Dórea et al. 2013b, Ohkusa et al. 2011, Shaman \& Karspeck 2012, Shaman et al. 2013). However, several weaknesses 
of these methods described in previous decades (Laporte 1993) have still not been addressed in the method designs. In addition, experiences from winter influenza seasons (Butler 2013) and the pandemic pH1N1 outbreak in 2009 (Santos-Preciado et al. 2009) suggest that existing information systems used for detecting and predicting epidemics and informing situational awareness have deficiencies when under heavy demand. Public health actions informed by forecasts that later turn out to be inaccurate can have negative effects on society, including loss of trust in authorities, misdirected resources, and, in the worst case, unnecessary morbidity or mortality. Unfortunately, influenza surveillance is one area where such forecasts have been contested (Lazer et al. 2014, Chretien et al. 2014). Subsequently, public health specialists seek more effective and equitable response systems, but methodological problems frequently limit the usefulness of novel approaches (Keller et al. 2009). In these biosurveillance systems, algorithms for detection and prediction of influenza epidemics are essential components (Timpka et al. 2011, Nsoesie et al. 2014).

Although a large number of studies have reported algorithms for influenza detection or prediction (Chreiten et al. 2014, Nsoesie et al. 2014, Buckeridge 2007, Hiller et al. 2013), they seldom produce output that fulfils standard criteria for operational readiness (Corley et al. 2014). For example, a recent review of influenza forecasting methods assessed studies that validated models against independent data (Chretien et al. 2014). Use of independent data is vital for predictive model validation, because using the same data for model fitting and testing inflates estimates of predictive performance (Hastie et al. 2009). The review concluded that the outcomes predicted and metrics used in validations varied considerably, which limited the possibility of formulating recommendations. It has also been pointed out that present prediction models have often been designed for particular situations using the data that are available and making assumptions where data are lacking (Louz et al. 2010, Neuberger et al. 2013).

An additional limitation in the public health field is that, although a large number of studies have presented potentially interesting algorithms for influenza surveillance, relatively few studies have compared the performance of different algorithms in routine practice using prospective designs. However, in a recently reported challenge program arranged by the Centers for Disease Control and Prevention (CDC 2013), nine research groups prospectively applied forecasting methods to four different aspects of 
epidemics (start week, peak week, peak percentage, and duration) using authentic routine data. Unfortunately, none of these methods generated satisfactory results for all four components (Biggerstaff et al. 2016). This implies that further research and development of integrated detection and prediction (nowcasting) methods that predict these kinds of aspects of epidemics are highly warranted in the public health field. Therefore, the first step is to gather studies that both develop and apply various types of influenza surveillance algorithms prospectively, with the aim of helping researchers to distinguish between the algorithms that display a satisfactory predictive performance and those that underperform when applied in natural settings. In other areas, such as meteorology, nowcasting methods have been used for decades (see e.g., Browning \& Collier 1989, Dixon \& Wiener 1993, Golding 1998) and are regarded as standard tools for warning the public against dangerous, high-impact events (Bližňák et al. 2017).

Moreover, in the light of the rapidly growing availability of "Big Data" from both diagnostic and pre-diagnostic (syndromic) sources in health care and public health settings, a new generation of epidemiological and statistical methods is desirable for reliable analyses and modeling (Riley et al. 2016). This need for new methods adapted to extensive, but heterogeneous, datasets extends to algorithms for detection and prediction of influenza epidemics. A pressing concern in the latter setting is that reports of methods for analyses of extensive datasets originating from different sources do not always meet basic scientific standards. In particular, the reports fail with regard to the requirement that researchers should be able to assess the design and performance of the methods when building the next generation of algorithms (Lazer et al. 2014). Regardless of the transparency problems in reporting, the potential of Big Data analyses in infectious disease control is widely recognized. In the past few years, a considerable amount of research has focused on the use of interactive health information technology - referred to as eHealth systems-to improve the effectiveness of infectious disease surveillance (Castillo-Salgado 2010). Researchers have focused on the use of Internet search engines (Kim et al. 2013, Sharpe et al. 2016, Pollett et al. 2017), telenursing data (Timpka et al. 2014a), mini-blogs (Nagel et al. 2013, YomTov et al. 2014, Sharpe et al. 2016), and records of over-the-counter drug sales (Kirian \& Weintraub 2010, Socan et al. 2012). This implies that the area where the need for knowledge is most immediate is the detection and prediction of influenza activity at local levels (Shaman et al. 2013). Before syndromic or digital data sources can be used in influenza surveillance systems, thorough 
research on how well these sources are related to local diagnostic sources is warranted. Identifying sources strongly associated with diagnostic data would allow use of these sources for monitoring, detecting, and predicting influenza activity. 


\section{AIMS}

The overall aim of this thesis was to develop an influenza nowcasting method that detects influenza epidemics in a satisfactory way and generates satisfactory predictions of the peak timing and the peak intensity. The rationale for developing the method was to inform the planning of local response measures and adjustments in health care capacity. A supporting aim was to perform a literature study of the detection and prediction methods currently used in this field and to compare these with each other in a prospective evaluation study. Another supporting aim was to find data sources other than the traditional ones (such as laboratory-verified influenza data and influenza-diagnosis data) and to use these as a complement to the traditional sources when detecting and/or predicting influenza epidemics and their characteristics.

The specific aims of the papers included in the thesis were:

- To perform a meta-narrative review of prospective evaluations of influenza epidemic detection and prediction algorithms. To ensure that the review results can be used to inform operational readiness, the scope was restricted to settings where authentic prospective surveillance data were used for the evaluation.

- Primarily to examine correlations between eHealth data sources (data from Google Flu Trends (GFT), computer-supported telenursing centers and health service websites) and influenza-diagnosis case rates during influenza epidemics. The secondary objective was to investigate associations between eHealth data, media coverage, and the interaction between circulating influenza strain(s) and the age-related population immunity.

- To perform a comparative trial of algorithms for the detection and prediction of influenza activity using local data from a county-wide public health information system using clinical influenza-diagnoses recorded by physicians and syndromic data from a telenursing service.

- To present a method for integrated nowcasting (detection and prediction) of influenza activity using data electronically available for real-time surveillance in local settings in the Western hemisphere, and to evaluate its performance by retrospective application on authentic data from a Swedish county.

- To perform a prospective 5-year evaluation of the previously reported influenza nowcasting method for application in local settings. The 
method includes three functions: detection of the start of the epidemic at local level and predictions of the peak timing and the peak intensity. 


\section{METHODS}

The research in this thesis involved methods for meta-analyses of the scientific literature, methods for the design of novel epidemiological procedures, and methods for evaluating these procedures in a structured way, which made it possible to answer the research questions corresponding to the thesis aims. The research consists of two phases reported in five papers: a preparatory phase, with two parts, and a development phase. To establish the potential of using eHealth data sources (such as GFT and telenursing data) to improve the effectiveness of infectious disease surveillance recognized by a large number of researchers, a study was conducted in the first part of the first phase to examine the associations between influenzadiagnosis data and eHealth data. In the analyses of associations, Pearson's correlation coefficients were examined to compare influenza-diagnosis rates with the eHealth data sources. GFT data and all possible combinations of telenursing chief complaints and website page visits with up to a 2-week time lag to influenza-diagnosis rates were examined.

In the second part of the preparatory phase, a broad review of studies prospectively evaluating algorithms for the detection or short-term prediction of influenza epidemics based on routinely collected data was performed using a meta-narrative review approach (Wong et al. 2013). This is a relatively new method of systematic analyses of published literature, designed for topics that have been conceptualized differently and studied by groups of researchers from different paradigms (Greenhalgh et al. 2005). The purpose of the meta-narrative review was to find studies that deal with methods of interest for this thesis and classify them into different categories, such as appropriate narratives, type of algorithms used, and temporal data used.

Following the findings in the meta-narrative review, an evaluation study of the algorithms found in the review was performed. This study applied an accuracy trial design (Bossuyt et al. 2012) based on two data streams used for routine influenza surveillance in a Swedish county (population 445,000): data on clinical influenza-diagnoses recorded by physicians and syndromic chief complaint data from a national telenursing service. The algorithms found in the meta-narrative review were, if applicable, evaluated in the comparative trial by applying them to these data streams. The evaluation was performed by splitting the two data streams into a learning dataset and an evaluation 
dataset; learning data were used to retrospectively decide parameter settings, which were then used to evaluate the detection and prediction algorithms prospectively using the evaluation dataset.

In the development phase, a nowcasting method for application in local influenza surveillance was defined. The method includes three functions: detection of the local start of the epidemic and predictions of the peak timing and the peak intensity. The resulting method was first applied retrospectively on authentic data from a Swedish county, and then evaluated on prospective data from the same county.

\section{Preparatory phase: examination of eHealth data sources}

The first part of the preparatory phase included a study examining the association between influenza-diagnosis cases and several potential eHealth data sources. The rationale for this study was to investigate the potential of using eHealth data sources for influenza surveillance in local settings.

\section{Performance of eHealth data sources in local influenza surveillance}

Study design

Based on the total population of Östergötland County $(n=445,000)$, an open cohort design was used in this study. Open cohort denotes that new cohort members are included by birth or by moving into the county and other members are excluded when passing away or moving out of the county as the cohort follow-up progresses. To update the open study cohort, annual aggregated data on the sex, age, and residence of the population in the county were collected for each year from Statistics Sweden. Personal identifiers were removed from the records, in accordance with Swedish legislation (SFS 2008:355). In this study, the start and end time of an influenza epidemic was defined as 2 influenza-diagnosis cases per 100,000 population recorded in the county over a floating 7-day period.

\section{Data collection}

Influenza case data, defined by clinical diagnoses, and eHealth data were collected between November 2007 and April 2012 using the electronic health data repository maintained by Östergötland County Council. For this study, data from the clinical laboratories were collected from 1 January 2009 to 15 
September 2010. Influenza-diagnosis cases were classified according to the ICD-10 codes for influenza (J10.0, J10.1, J10.8, J11.0, J11.1, and J11.8). Telenursing calls were identified by the chief complaint codes associated with influenza symptoms: dyspnea, fever (child, adult), cough (child, adult), sore throat, lethargy, syncope, dizziness, and headache (child, adult), from the fixed-field terminology register. GFT data were collected using a Google account to download data on Google searches from Östergötland County on seasonal influenza and the 2009 pandemic outbreak to a database. GFT did not consist of absolute search rate data, but consisted of influenza Web search data normalized with regard to total Web search volumes by the GFT software. Usage data from the county council webpages were collected at the beginning of May 2009. Because software providers changed, these data could not be retrieved for the 2010-11 winter influenza season. From January 2012, usage data for the Web-based information service, measured by the numbers of visits to a certain type of page, were collected from the instances of Google Analytics Web traffic analysis. Page type refers to the kind of content in the page; e.g., factual information about influenza, commonly asked questions and answers, information on self-care, or news pages. Data on media coverage related to influenza were collected from the online database of the largest newspaper in the county (Östgöta Correspondenten). Articles with the term "influenza" (influensa in Swedish) was searched for in the database for the period between November 2007 and April 2012.

\section{Data analysis}

The influenza-diagnosis case data were validated against laboratory-verified influenza case data for the period from 1 January 2009 to 15 September 2010. In these analyses, the datasets were adjusted separately for weekday effects on utilization of care resources. The associations (in terms of Pearson's correlation coefficient) between the number of influenza-diagnosis cases and laboratory-verified cases were analyzed with a lag of 0-6 days.

To compare the relative distribution of influenza-diagnosis cases between age groups, the relative illness ratio (RIR) was calculated for each age group and epidemic (circulating virus type). RIR is defined as the ratio between the percentage of individuals diagnosed with influenza in a given age group and the percentage of the general population belonging to the same age group and is computed using the formula

$$
R I R_{i}=\frac{C_{i} /_{C}}{N_{i} /_{N}}
$$


where $C_{i}$ denotes the number of influenza-diagnosis cases in age group $i, C$ denotes the total number of influenza-diagnosis cases, $N_{i}$ represents the population in age group $i$, and $N$ represent the total population in the county. Furthermore, using a method based on normal approximation of the Poisson distribution, 95\% confidence intervals were calculated for each RIR.

In the main analyses of associations between data on influenza-diagnoses and eHealth data, Pearson's correlation coefficients $(r)$ were examined to compare the case rates of influenza-diagnoses with the eHealth data sources, i.e., GFT data and all possible combinations of telenursing chief complaints and website page visits. Furthermore, the correlation analyses were performed with a time lag of up to 2 weeks between eHealth data and case rates of influenza-diagnoses (eHealth data preceding data on influenza-diagnosis cases). The three groupings of chief complaints and combinations of website page types with the strongest correlation to influenza-diagnosis cases for each time lag were listed. In the final analyses, only the chief complaint grouping and website page combination with the largest correlation effect size were used. Analyses of correlations between media reports, influenzadiagnosis data, and the eHealth data sources, were also performed with a time lag of up to 2 weeks to media reports. The level of statistical significance was set to $P<0.05$. To denote the correlation strengths, limit values suggested by the Cohen scale (Cohen 1988) were used. This scale defines small, medium, and large effect sizes as $0.10,0.30$, and 0.50 , respectively.

\section{Preparatory phase: review and evaluation of existing detection and prediction algorithms}

The second part of the preparatory phase include two papers: a metanarrative review of studies evaluating detection and prediction algorithms and a comparative trial evaluating these algorithms.

\section{Meta-narrative review and comparative trial}

\section{Study design}

In the first study of the second part of the preparatory phase, a meta-narrative review (Wong et al. 2013) was conducted to assess publications that prospectively evaluated algorithms for detection or short-term prediction of influenza epidemics using routinely collected data. This approach is suitable for addressing the question "what works?" and to clarify a complex topic, 
underlining the strengths and limitations of different research approaches to that topic (Mays et al. 2005), and was therefore chosen in this study. Metanarrative reviews look at how specific research traditions have unfolded historically, how the type of questions being asked are formed, and the methods used to answer them. The range of approaches to study a particular issue are examined, an account of the development of these separate metanarratives is interpreted and produced, and a central meta-narrative summary is formed. The principles of pragmatism (inclusion criteria are guided by what is considered to be useful to the audience), pluralism (the topic is illuminated from multiple perspectives; only research that lacks rigor is rejected), historicity (research traditions are described as they unfold over time), contestation (conflicting data are examined to generate higher order insights), reflexivity (reviewers continually reflect on the emerging findings), and peer review were applied in the analysis (Wong et al. 2013). Four steps were taken in the meta-narrative review: an electronic literature search was performed, publications were selected, data from these papers were extracted, and qualitative and semiquantitative analyses of the content were performed. For extraction of data and analyses, researcher triangulation (including several researchers with diverse backgrounds and qualifications) was used as a strategy to ensure the quality. All steps were documented and managed electronically using a database.

For an evaluation study to be included in the review, it had to apply an influenza detection or prediction algorithm to authentic data prospectively collected to detect or predict naturally occurring influenza epidemics among humans. Following the inclusive approach of the meta-narrative review methodology, studies using clinical influenza-diagnoses and laboratoryverified influenza-diagnoses for case verification were included (Unkel et al. 2012). For the evaluations of the prediction algorithms, correlation analyses were also accepted, because interventions could have been implemented during the evaluation period. In addition, studies were required to compare syndromic data with some gold standard data from known influenza epidemics. All studies published from 1 January 1998 to 31 January 2016 were considered.

In the second study of the second part of the preparatory phase, an accuracy trial design (Bossuyt et al. 2012) was applied. Two streams of data used for routine influenza surveillance in Östergötland County, Sweden (population 445,000 inhabitants) were used in this study: data on clinical influenza- 
diagnoses and data on syndromic chief complaints from a national telenursing service. The latter data source had previously been found to provide indications of increased influenza activity up to 2 weeks ahead of the former (Timpka et al. 2014a, 2014b).

The algorithms found in the meta-narrative review were evaluated in the comparative trial. Therefore, the primary criteria for inclusion in this study was the same as in the meta-narrative review: an algorithm had to have been evaluated using authentic data prospectively collected to detect or predict naturally occurring human influenza epidemics, and the report had to have been published in a peer-reviewed scientific journal between 1 January 1998 and 31 January 2016. However, in the comparative trial, four secondary criteria were added. The first was that the algorithm was applicable for county-level influenza surveillance, i.e., on unidimensional influenza data or syndromic data associated with influenza from a population of approximately 500,000 inhabitants. The second and third secondary criteria were that the algorithm had been sufficiently documented to be reproduced and that it could be calibrated using a maximum of 1 season of learning data. The final secondary criterion was that the detailed assumptions about the data features were compatible with the county-level data used for the evaluation.

\section{Meta-narrative review: data collection and analysis}

In February 2016, PubMed was searched for possible relevant papers to be included in the review using the following search term combinations: "influenza AND ((syndromic surveillance) OR (outbreak detection OR outbreak prediction OR real-time prediction OR real-time estimation OR realtime estimation of $\mathrm{R}))^{\prime \prime}$. Only papers and book chapters available in English were considered for inclusion and further analysis. To describe the features of the papers meeting the inclusion criteria, information was documented regarding the main objective, the publication type, the algorithm applied, the application context, whether syndromic data were used, and country.

Information about the papers was analyzed semiquantitatively by grouping papers with equal or similar features and by counting the number of papers in each group. In the following step, paragraphs or sentences containing key terms (such as study aims, description of the algorithm, or the context of the application) were extracted and documented in the database. The 
documentation of data from the papers and extraction of text were performed by one reviewer; a second reviewer critically rechecked the documented data. In the next step, content analysis of the extracted text was conducted and the meaning of the original text was condensed. The condensed statements contained as much information as required to adequately represent the meaning of the text in relation to the research aim. However, they were as short and simple as possible to allow straightforward processing. If the original text contained several pieces of information, a separate statement was created for each piece of information. A coding scheme was established inductively to analyze the information contained in the papers. Condensed statements could be labeled with more than one code. One reviewer created the condensed statements and their coding, and a second reviewer rechecked these. The preliminary versions were compared and agreed upon, which resulted in final versions of the condensed statements and their coding. Information about the detection and prediction algorithms was summarized qualitatively in tables and analyzed semiquantitatively based on this coding. The next part of the analysis phase involved identification of the key dimensions of the algorithm evaluations, providing a narrative account of the contribution of each dimension and explaining conflicting findings. The narratives identified in the meta-narrative review are presented using descriptive statistics and narratively without quantitative pooling. In the final step, a wider research team and policy leaders $(n=11)$ with backgrounds in public health, social sciences, computer science, cognitive science, and statistics were involved in a process where the findings were tested against their experiences and expectations. The feedback from these policy leaders and researchers was used for further reflection and analysis. The final report was compiled after this feedback.

A semantic system was introduced to enable interpretation of the performance of the algorithms identified in the meta-narrative review. Values for the area under the curve (AUC) were set at 0.90, 0.80, and 0.70, representing outstanding, excellent, and acceptable discriminatory performance, respectively (Hosmer \& Lemeshow 2000). The same limits were used to interpret the performance of the area under the weighted receiver operating characteristic curve (AUWROC) and the volume under the time receiver operating characteristic surface (VUTROC) metrics. The limits for sensitivity (defined as the proportion of correctly identified days/weeks with increased influenza activity), specificity (defined as the proportion of correctly identified days/weeks with no increased influenza activity), and 
positive predictive value (PPV) were set at $0.95,0.90$, and 0.85 , respectively, for analyses of weekly data, and 0.90, 0.85 and 0.80 for analyses of daily data, representing outstanding, excellent, and acceptable performance, respectively. To interpret the correlation strengths, limits were modified from the Cohen scale (Cohen 1988); the limits 0.10, 0.30, and 0.50 were defined as small, medium, and large effect sizes, respectively. In the meta-narrative review, the limits were set at $0.90,0.80$, and 0.70 when weekly data were analyzed, and $0.85,0.75$, and 0.65 when daily data were analyzed, denoting outstanding, excellent, and acceptable predictive performance, respectively. The limit values are summarized in Table 1.

Table 1. Summary of the semantic system used to interpret algorithm performance

\begin{tabular}{|c|c|c|c|}
\hline \multirow[t]{2}{*}{ Measurement } & \multicolumn{3}{|l|}{ Performance } \\
\hline & Outstanding & Excellent & Acceptable \\
\hline \multicolumn{4}{|l|}{ Epidemic detection and prediction } \\
\hline AUC, AUWROC, VUTROC & 0.90 & 0.80 & 0.70 \\
\hline Sensitivity, specificity, PPV (weekly) & 0.95 & 0.90 & 0.85 \\
\hline Sensitivity, specificity, PPV (daily) & 0.90 & 0.85 & 0.80 \\
\hline \multicolumn{4}{|l|}{ Only epidemic prediction } \\
\hline Pearson's correlation (weekly) & 0.90 & 0.80 & 0.70 \\
\hline Pearson's correlation (daily) & 0.85 & 0.75 & 0.65 \\
\hline
\end{tabular}

AUC, area under curve; AUWROC, area under weighted ROC curve; VUTROC, volume under the timeROC surface; PPV, positive predictive value.

\section{Data sources used in the comparative accuracy trial}

The data used in the comparative trial were collected from the electronic health data system maintained by Östergötland County Council (Timpka et al. 2011). This system assembles data from all patient visits at health care centers in the county and from calls made by the county residents to the nation-wide telenursing service. The same influenza-diagnosis codes and telenursing chief complaints used in the first step of the preparatory phase were used in this study, i.e., influenza ICD-10 codes J10.0, J10.1, J10.8, J11.0, J11.1, and J11.8 and telenursing chief complaints of dyspnea, fever (child, adult), cough (child, adult), sore throat, lethargy, syncope, dizziness, and headache (child, adult). The learning dataset used to calibrate the algorithms covered the winter influenza season of 2008-09, starting from the end of the previous winter influenza season. The evaluation period started immediately after the end of the learning period, covering the pandemic outbreak in 2009 and the two winter influenza seasons of 2010-11 and 2011-12 (Figure 1). Since the evaluation period contained both a pandemic outbreak and winter influenza seasons, it was divided into two parts when performing the 
analyses: one part covered the pandemic and the other part covered the two winter influenza seasons. In the comparative trial, the epidemic threshold was defined as 2 incident influenza-diagnosis cases per 100,000 population recorded during a 7-day period. Data on a weekly basis were used in the analyses of this study.
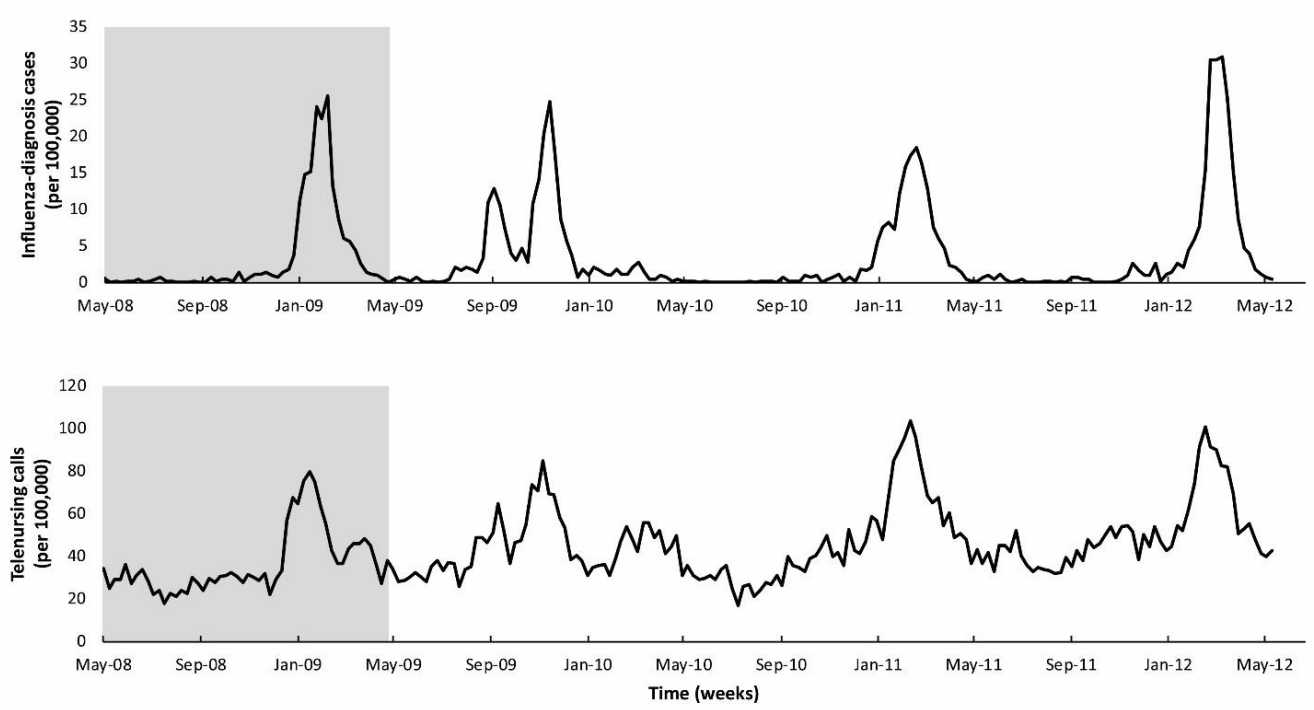

Figure 1. Weekly rates of influenza-diagnosis cases and telenursing calls for fever (child, adult) in Östergötland County, Sweden, during the retrospective learning period from May 2008 to April 2009 (the gray shaded area) and the prospective evaluation period from April 2009 to May 2012.

\section{Evaluation procedure in the comparative trial}

For influenza-diagnosis data, parameter settings for the different detection and prediction methods were set retrospectively using the learning dataset. For telenursing data, the time lag and grouping of the chief complaints most strongly associated with influenza-diagnosis data were determined first. The combination of chief complaint grouping and time lag with the largest correlation strength was chosen as a "new" learning set for telenursing data. The new learning set was then used to determine parameter settings for the different detection and prediction methods. When the parameters for the two data streams (influenza-diagnosis data and telenursing data) had been decided, they were first re-applied in retrospective analyses using the learning dataset, and then applied in prospective analyses using the evaluation dataset. 
The metrics used to evaluate the performance of the detection algorithms were sensitivity, specificity, and timeliness (defined as the time difference between the observed and the predicted start of a period with increased influenza activity). To evaluate the performance of the prediction algorithms, Pearson's correlation coefficient $(r)$ and median absolute percentage error (MedAPE), both representing the association between the observed and the predicted time series of influenza activity, were used.

The performance of the detection algorithms in the comparative trial was considered acceptable if the specificity was at least 0.85 and the sensitivity was at least 0.80 . The limit of the sensitivity differed slightly in the comparative trial compared with the meta-narrative review (see Table 1). The reason for adjusting the sensitivity limit in the comparative trial was to give specificity priority over sensitivity because a high level of false alarms is unacceptable in public health practice. If several algorithms performed equally with regard to specificity and sensitivity, timeliness was used to decide which of the algorithms was superior. Specificity was calculated using the 10 weeks immediately before an epidemic, and sensitivity was calculated using the first 10 weeks of an epidemic. The reason why these measures were not based on entire datasets was that detection methods are primarily optimized to detect epidemics. Extending the periods on which the calculations are based would yield an overestimation of these metrics, which would result in a misleading performance of the algorithm.

For the evaluation of the prediction algorithms, Pearson's correlation coefficient $(r)$ was used as the primary metric of the association between observed and predicted values. To interpret the correlation strengths, limits were set at $0.90,0.80$, and 0.70 denoting outstanding, excellent, and acceptable predictive performance (Table 1). The secondary evaluation metric, MedAPE, is an accuracy measure of fitted time series values (Burkom et al. 2007). For a perfect fit, MedAPE is zero with no upper level restriction. MedAPE gives an idea of the typical percentage error and enables comparisons across different series. The combination of Pearson's correlation and absolute percentage error (MedAPE before the median is calculated) has been used previously (Jiang et al. 2009).

\section{Algorithms evaluated and excluded in the comparative trial}

Seven influenza detection algorithms were found to have been evaluated using authentic prospective data: an algorithm based on the Kolmogorov- 
Smirnov test, a time series method based on a dynamic model, two hidden Markov models, a CUSUM algorithm, a simple regression model and a Serfling regression model. However, only the latter three were evaluated in the comparative accuracy trial because the other algorithms failed to meet the secondary inclusion criteria of this study.

Regarding prediction algorithms, nine were found to have been evaluated in prospective settings using authentic data: a Shewhart-type model, a multiple linear regression model, a Bayesian network model, a linear autoregressive model, the Holt-Winters method, the method of analogues, a naive method, a nonadaptive log-linear regression model, and an adaptive log-linear regression model. Only the latter three met the secondary inclusion criteria of the comparative trial and thus only they were evaluated.

Detailed descriptions of the evaluated algorithms as well as reasons for exclusion of the algorithms not meeting the study criteria are provided in the Results section.

\section{Development phase: design and evaluation of the nowcasting method}

This section describes the development phase and includes the design of a nowcasting method and a prospective evaluation of the method. The nowcasting method consists of three components: epidemic detection and prediction of the peak timing and peak intensity of the epidemic. The resulting method was first applied retrospectively on authentic local data from a county-wide public health information system, followed by a prospective evaluation using the same data sources.

\section{Study design and design rationale}

The rationale for design of the particular integrated nowcasting method is that the aim of local influenza surveillance is early detection and prediction of infected individuals requiring clinical attention. The main purpose of this is timely allocation of health care resources. Since precious time is lost before laboratory-verified data are available for algorithmic processing and laboratory test samples are not taken from all patients, influenza-diagnosis data are superior as gold standard data. Furthermore, due to that it is theoretically challenging to only use unidimensional gold standard data to predict the peak timing, syndromic data are used for this purpose. 
The detection function and the prediction functions must comply with the required quality and accuracy criteria for technologies to be applied in health care and public health practice (Thokala et al. 2016). The theoretical assumptions underpinning the design of the detection module are that the number of influenza-diagnosis cases grows exponentially at the start of periods of increased activity and that an alerting threshold can be set by using historical data from previous winter influenza seasons. For peak timing predictions, evidence of a strong association between the gold standard and syndromic data sources used for influenza surveillance is assumed to be available. Regarding the peak intensity predictions, the peak timing is assumed to be previously determined and the number of influenza-diagnosis cases is assumed to follow a bell-shaped function of time around the peak. Based on these assumptions, the nowcasting method was developed and evaluated using an open cohort design based on the total population $(n=445,000)$ in Östergötland County, Sweden.

\section{Data sources}

The same two data streams as in the comparative accuracy trial were used in the development phase of this thesis: influenza-diagnosis data and syndromic telenursing chief complaint data. The study period covered data from 1 January 2008 to 30 June 2014. The data were divided into a learning set and an evaluation set. The learning dataset, used to calibrate the nowcasting method, ranged from 1 January 2008 to 30 June 2009 and contained the two winter influenza seasons of 2007-08 and 2008-09. The evaluation dataset, used to evaluate the performance of the nowcasting method, ranged from 1 July 2009 to 30 June 2014 and contained the 2009 pandemic outbreak and the four winter influenza seasons of 2010-11, 201112, 2012-13 and 2013-14.

\section{Definitions}

Influenza detection is defined as the beginning of an epidemic in the community, i.e., when an extended period of increased incidence rates (exceeding a pre-defined limit) of influenza-diagnosis cases has occurred. Influenza prediction denotes foretelling the peak timing and the peak intensity of an epidemic in the community.

The influenza-diagnosis case rate when a local influenza epidemic factually takes off was set to 6.3 influenza-diagnosis cases/100,000 over a floating 7-day period. This limit was determined by inspecting the epidemic curves of 
previous local influenza epidemics in the learning dataset. In a recent comparison of influenza intensity levels in Europe (Vega et al. 2015), a similar definition (6.4 influenza-diagnosis cases/week/100,000) was estimated for the 2008-09 winter influenza season in Sweden. The definition of when an epidemic ends was set to the inter-epidemic (i.e., the period between two epidemics) influenza-diagnosis case level for the setting where the nowcasting method is applied. This definition was necessary because the detection algorithm requires the influenza activity to be at an inter-epidemic level before the algorithm can begin with the influenza activity scan. The peak timing was defined to occur on the date when the highest number of influenza-diagnosis cases were documented in the county-wide electronic patient records; the peak intensity was defined as the number of influenzadiagnosis cases documented on that date.

\section{Calibration of the nowcasting method}

To calibrate the detection component of the nowcasting method, weekday effects of influenza-diagnosis cases (Donker et al. 2011) and an optimal baseline alarm threshold were determined retrospectively. The learning dataset, including the two winter influenza seasons of 2007-08 and 2008-09, was used to calculate the weekday effects, whereas only the winter influenza season in 2008-09 was used to determine the initial alerting threshold. The reason for not using the 2007-08 winter influenza season for the latter purpose was because the collection of the learning dataset was initiated when this season had already begun. The alerting threshold was updated after every winter influenza season (i.e., no updates after the pandemic outbreak), using data from all available previous winter influenza seasons throughout the study period. In other words, the detection algorithm was applied to the forthcoming epidemic using the revised threshold determined in the updated learning dataset (covering all previous winter influenza seasons).

The learning dataset including the two winter influenza seasons in 2007-08 and 2008-09 was also used to initially calibrate the peak timing prediction component. Data from this learning set were used to determine the grouping of telenursing chief complaints with the largest correlation strength and best lead time between influenza-diagnosis data and telenursing data. The optimal combination of chief complaints was found to be fever (adult, child), and the most favorable lead time was 14 days (with telenursing data preceding influenza-diagnosis data) (Timpka et al. 2014a, 2014b). Using the predicted peak timing, the peak intensity prediction component was applied 
to influenza-diagnosis data from the corresponding epidemics to estimate the peak intensity on the predicted peak day.

The same weekday effects were used throughout the evaluation study both in local detection and local prediction analyses because they were assumed to be relatively constant over time. The same applies also to the optimal grouping of telenursing chief complaints and optimal lead time (used only in the local prediction analyses).

\section{Application of the nowcasting method}

In the retrospective evaluation of the performance of the nowcasting method, the method was first re-applied to the 2008-09 winter influenza season using the alerting threshold and weekday effects determined in the learning set (which contained this winter influenza season). In the prospective performance evaluation of the nowcasting method, the method was prospectively applied to each epidemic included in the evaluation dataset, i.e., the pandemic outbreak in 2009 and the four following winter influenza seasons.

\section{Metrics and interpretation}

The metrics used to determine the optimal alerting threshold of the detection function were sensitivity and specificity. Specificity was calculated from when the detection algorithm started its search (i.e., when the previous epidemic ended) and until the current epidemic began according to the standard definition (6.3 influenza-diagnosis cases/100,000 over a floating 7day period). This means that the period on which the specificity calculation is based varies with the inter-epidemic period. Regarding sensitivity, the calculation was based on the first 45 days of an epidemic. The optimal threshold was set by calculating the sensitivity and specificity and studying them in a receiver operating characteristic (ROC) curve. In addition to sensitivity and specificity, timeliness, defined as the time difference in days between the actual start of the epidemic and the start indicated by the model, was used as a metric to evaluate the performance of the detection component in the retrospective setting. Timeliness, defined as the time difference between the predicted day of the influenza-diagnosis peak and the peak day in the observed smoothed series of influenza-diagnosis data, was also used to evaluate the performance of the peak timing prediction function. To evaluate the performance of the peak intensity prediction function, the 
absolute and relative differences between the predicted peak intensity and observed peak intensity were used.

In the prospective evaluation, timeliness was once again used to evaluate the performance of the detection and peak timing prediction functions, and the correct identification of the intensity category on a five-point scale was used to evaluate the performance of the peak intensity function. For trustworthiness of the nowcasting method in local health care planning, the maximum acceptable timeliness error in detection and peak timing predictions was set to 1.5 weeks. The performance of the two functions was defined as excellent if the Itimeliness error $\mid$ was $\leq 3$ days, good if it was between 4 and 7 days, acceptable if it was between 8 and 11 days and poor if the I timeliness error $\mid$ was $\geq 12$ days. To evaluate the performance of the peak intensity function, the epidemic threshold and intensity level categories (nonepidemic, low, medium, high, and very high) were used (Table 2). These categories and their limits were determined in a recent comparison of influenza intensity levels in 28 European countries, including Sweden (Vega et al. 2015). A prediction was considered successful if the predicted peak intensity fell into the same category as the actual peak intensity, otherwise the prediction was considered unsuccessful.

Table 2. Epidemic intensity categories used for interpretation of performance measurements

\begin{tabular}{l|cccccc}
\hline $\begin{array}{l}\text { Threshold } \\
\text { (cases/day/100,000 }\end{array}$ & \multicolumn{7}{|l}{ Influenza season } \\
\cline { 2 - 7 } population) & $\mathbf{2 0 0 8 - 0 9}$ & $\begin{array}{c}\text { Pandemic } \\
\mathbf{2 0 0 9}\end{array}$ & $\mathbf{2 0 1 0 - 1 1}$ & $\mathbf{2 0 1 1 - 1 2}$ & $\mathbf{2 0 1 2 - 1 3}$ & $\mathbf{2 0 1 3 - 1 4}$ \\
\hline Non-epidemic level & $<0.9$ & $<0.9$ & $<0.9$ & $<1.0$ & $<1.2$ & $<1.2$ \\
\hline Low intensity & 0.9 & 0.9 & 0.9 & 1.0 & 1.2 & 1.2 \\
\hline Medium intensity & 2.4 & 2.5 & 2.5 & 2.5 & 2.8 & 2.9 \\
\hline High intensity & 5.5 & 5.4 & 5.4 & 5.2 & 5.6 & 5.5 \\
\hline Very high intensity & 7.9 & 7.5 & 7.5 & 7.1 & 7.7 & 7.4 \\
\hline
\end{tabular}




\section{RESULTS}

\section{Preparatory phase: examination of eHealth data}

\section{sources}

\section{Overview}

The results from the validation analyses showed correlations with large effect sizes between the daily number of influenza-diagnosis cases and the corresponding daily number of laboratory-verified cases during the validation period. The correlation with largest effect size $(r=0.63, P<0.001)$ was observed between these two data streams with a 2-day lag.

The 5-year study period covered the four winter influenza seasons of 2007-08 (B and A(H1)), 2008-09 (A (H3N2)), 2010-11 (B and A (pH1N1)), 2011-12 (A (H3N2)), and the pandemic outbreak in 2009 (A (pH1N1)). Higher than expected proportions of influenza-diagnosis cases occurred in the middleaged groups (30-39 and 40-49 years) during all epidemics, whereas lower than expected proportions of influenza-diagnosis cases were recorded among adolescents and young adults (10-19 and 20-29 years) for those winter influenza seasons when the pandemic A (pH1N1) virus was not circulating (Figure 2). 

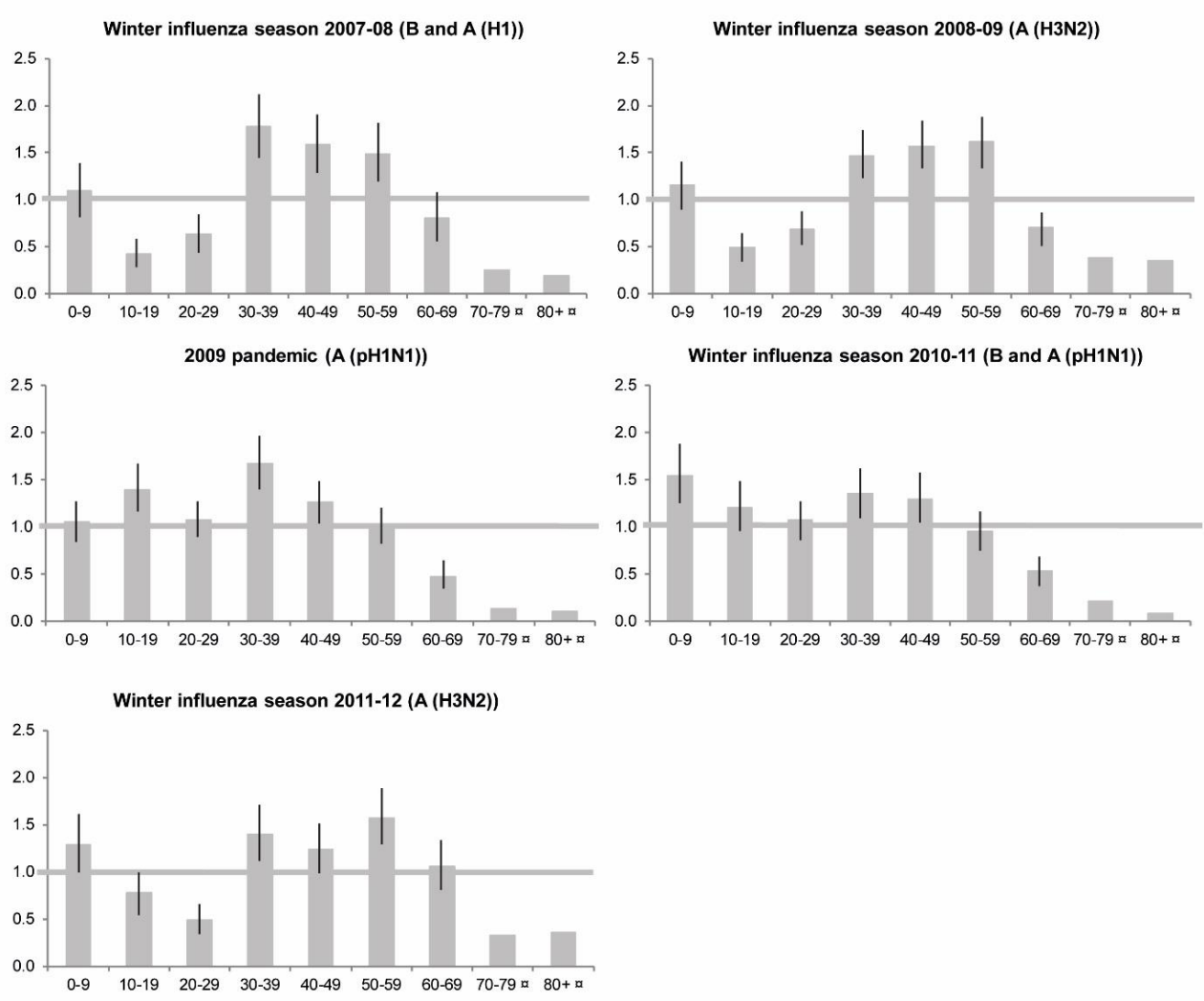

Figure 2. Relative infection ratios (RIRs) with 95\% confidence intervals for influenza epidemics between 2007 and 2012 in Östergötland County displayed by decennial age groups. $\not$ Too few observations to allow statistical analysis.

\section{Correlations between local media coverage, influenza- diagnosis cases, and eHealth data}

The correlations between local media coverage data and influenza-diagnosis rates showed only large effect sizes for the A ( $\mathrm{pH} 1 \mathrm{N1}$ ) pandemic outbreak in 2009 ( $r=0.74,95 \%$ CI 0.42-0.90; $P<0.001)$, and the severe seasonal A (H3N2) epidemic in 2011-12 ( $r=0.79,95 \%$ CI 0.42-0.93; $P=0.001)$, with media coverage data preceding influenza-diagnosis data by 1 week in both cases. In addition, media reports about influenza showed a peak for weeks 18-22 of 2009, which coincided with a sharp increase in GFT activity, but these peaks had no correspondence with influenza-diagnosis case rates or telenursing data (Figure 3). The correlations between media coverage and GFT displayed large effect sizes for the winter influenza seasons in 2008-09 ( $r=0.62,95 \%$ CI 0.15$0.86 ; P=0.014)$ and $2011-12(r=0.77,95 \%$ CI $0.39-0.93 ; P=0.002)$, as well as the 
pandemic outbreak in 2009 ( $r=0.69,95 \%$ CI 0.35-0.87; $P=0.001)$. The strongest correlations were found for no time lag except for the winter influenza season in 2012, when GFT activity preceded media coverage by 1 week. Neither telenursing data nor the data from health service provider webpages showed statistically significant associations with the local media coverage data.
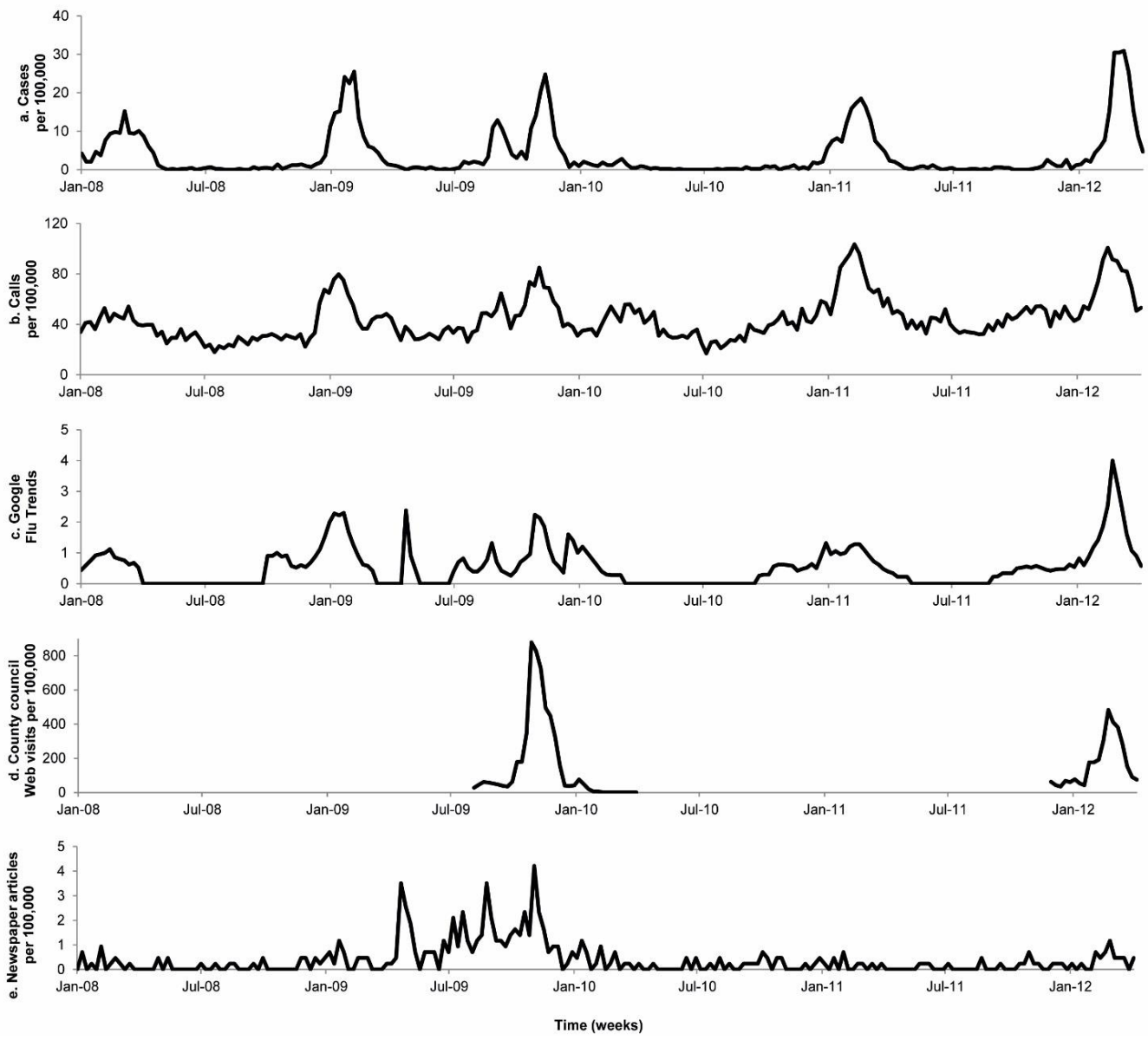

Figure 3. Display of (a) weekly rates of influenza-diagnosis cases, (b) weekly rates of telenursing calls for indicator chief complaints (fever and syncope), (c) Google Flu Trends output, (d) Influenza-specific website usage at local health service provider, and (e) articles mentioning influenza in a major regional newspaper. All data were collected from Östergötland County, Sweden, from November 2007 to April 2012.

\section{Correlations between GFT and influenza-diagnosis data}

The correlations between GFT and influenza-diagnosis data showed large effect sizes for all epidemics, varying between $r=0.69$ (95\% CI $0.22-0.90$ ), $P=0.010$, for the B and A (H1) winter influenza season in 2007-08 to $r=0.96$ 
(95\% CI 0.88-0.99), $P<0.001$, for the A (H3N2) winter influenza season in 200809 (Table 3). The time lag between GFT and influenza-diagnosis case rates was 1 week during the 2009 A (pH1N1) pandemic and the last winter influenza season, and 2 weeks for the other three winter influenza seasons, with GFT preceding influenza-diagnosis cases during all five epidemics.

Table 3. Associations on a weekly basis between GFT data and influenza-diagnosis data displayed by the correlation coefficient $r$ (95\% confidence interval) for the five influenza epidemics observed in Östergötland County, Sweden, during the study period 2007-12.

\begin{tabular}{|c|c|c|c|c|c|}
\hline $\begin{array}{l}\text { Epidemic } \\
\text { time lag } \\
\text { duration } \\
\text { (weeks) }\end{array}$ & $\begin{array}{l}2007-08 \\
\text { (B and A (H1)) } \\
\text { (15 weeks) }\end{array}$ & $\begin{array}{l}2008-09 \\
\text { (A (H3N2)) } \\
\text { (15 weeks) }\end{array}$ & $\begin{array}{l}2009 \\
\text { (A (pH1N1)) } \\
\text { (19 weeks) }\end{array}$ & $\begin{array}{l}\text { 2010-11 } \\
\text { (B and A } \\
\text { (pH1N1)) } \\
\text { (18 weeks) }\end{array}$ & $\begin{array}{l}2011-12 \\
\text { (A (H3N2)) } \\
\text { (14 weeks) }\end{array}$ \\
\hline 0 & n.s. & $\begin{array}{l}0.66 \\
(0.23,0.88) \\
P=0.007\end{array}$ & $\begin{array}{l}0.79 \\
(0.53,0.92) \\
P<0.001\end{array}$ & $\begin{array}{l}0.57 \\
(0.14,0.82) \\
P=0.013\end{array}$ & $\begin{array}{l}0.83 \\
(0.54,0.95) \\
P<0.001\end{array}$ \\
\hline 1 & n.s. & $\begin{array}{l}0.86 \\
(0.61,0.96) \\
P<0.001\end{array}$ & $\begin{array}{l}0.92 \\
(0.79,0.97) \\
P<0.001\end{array}$ & $\begin{array}{l}0.75 \\
(0.42,0.90) \\
P=0.001\end{array}$ & $\begin{array}{l}0.95 \\
(0.83,0.98) \\
P<0.001\end{array}$ \\
\hline 2 & $\begin{array}{l}0.69 \\
(0.22,0.90) \\
P=0.010\end{array}$ & $\begin{array}{l}0.96 \\
(0.88,0.99) \\
P<0.001\end{array}$ & $\begin{array}{l}0.69 \\
(0.31,0.88) \\
P=0.002\end{array}$ & $\begin{array}{l}0.81 \\
(0.53,0.93) \\
P<0.001\end{array}$ & $\begin{array}{l}0.83 \\
(0.50,0.95) \\
P=0.001\end{array}$ \\
\hline
\end{tabular}

Time lag 1 week = Influenza-diagnosis cases 1 week time shift, i.e., people first Google the terms "influenza" or "swine flu" and 1 week later visit the health services.

Time lag 2 weeks = Influenza-diagnosis cases 2 week time shift, i.e., people first Google the terms "influenza" or "swine flu" and 2 weeks later visit the health services.

n.s., not statistically significant.

\section{Correlations between telenursing call data and influenza- diagnosis data}

The correlations between telenursing data and influenza-diagnosis data displayed large effect sizes for all epidemics except for the B and A (H1) winter influenza season in 2007-08. The preceding time lag with the highest correlation was 2 weeks for the A (H3N2) winter influenza season in 2008-09 and 1 week for the two latest winter influenza seasons examined, with telenursing cases preceding influenza-diagnosis cases (Table 4). For the A ( $\mathrm{pH} 1 \mathrm{N1}$ ) pandemic outbreak, the highest correlation between these two data streams corresponded in time (i.e., the time lag was 0 weeks). During most of the epidemics, the combination of telenursing chief complaints displaying correlations with largest effect size to influenza-diagnosis cases were fever (child, adult) and syncope. 
Table 4. Associations on a weekly basis between telenursing call data and influenzadiagnosis data displayed by the correlation coefficient $r$ (95\% confidence interval) for the five influenza epidemics observed in Östergötland County, Sweden, during the study period 2007-12.

\begin{tabular}{|c|c|c|c|c|c|}
\hline $\begin{array}{l}\text { Epidemic } \\
\text { time lag } \\
\text { duration } \\
\text { (weeks) }\end{array}$ & $\begin{array}{l}2007-08 \\
\text { (B and A (H1)) } \\
\text { (15 weeks) }\end{array}$ & $\begin{array}{l}2008-09 \\
\text { (A (H3N2)) } \\
\text { (15 weeks) }\end{array}$ & $\begin{array}{l}2009 \\
\text { (A (pH1N1)) } \\
\text { (19 weeks) }\end{array}$ & $\begin{array}{l}2010-11 \\
\text { (B and A } \\
\text { (pH1N1)) } \\
\text { (18 weeks) }\end{array}$ & $\begin{array}{l}2011-12 \\
\text { (A (H3N2)) } \\
\text { (14 weeks) }\end{array}$ \\
\hline 0 & n.s. & n.s. & $\begin{array}{l}0.84 \\
(0.62,0.94) \\
P<0.001\end{array}$ & $\begin{array}{l}0.91 \\
(0.77,0.97) \\
P=0.001\end{array}$ & $\begin{array}{l}0.90 \\
(0.70,0.97) \\
P=0.008\end{array}$ \\
\hline 1 & n.s. & $\begin{array}{l}0.81 \\
(0.48,0.94) \\
P=0.001\end{array}$ & $\begin{array}{l}0.80 \\
(0.52,0.92) \\
P<0.001\end{array}$ & $\begin{array}{l}0.95 \\
(0.86,0.98) \\
P<0.001\end{array}$ & $\begin{array}{l}0.97 \\
(0.91,0.99) \\
P<0.001\end{array}$ \\
\hline 2 & n.s. & $\begin{array}{l}0.95 \\
(0.82,0.98) \\
P<0.001\end{array}$ & n.s. & $\begin{array}{l}0.88 \\
(0.69,0.96) \\
P=0.001\end{array}$ & $\begin{array}{l}0.93 \\
(0.77,0.98) \\
P=0.002\end{array}$ \\
\hline
\end{tabular}

Time lag 1 week = Influenza-diagnosis cases 1 week time shift, i.e., people first call Health care Direct/1177 and 1 week later visit the health services.

Time lag 2 weeks = Influenza-diagnosis cases 2 week time shift, i.e., people first Health care Direct/1177 and 2 weeks later visit the health services.

n.s., not statistically significant.

\section{Correlations between log data from the county council website and influenza-diagnosis data}

For the 2009 A (pH1N1) pandemic, the correlation with the largest effect size between log data from the county council website and influenza-diagnosis cases was noted for the website section "Influenza self-care and treatment" with no time lag $(r=0.75,95 \%$ CI $0.45-0.90 ; P=0.004)$, whereas the correlation with 1 week preceding lag (website visit preceding health care visit) for data from the section "Influenza facts" was only slightly smaller $(r=0.74,95 \% \mathrm{CI}$ 0.42-0.90; $P=0.006$ ). During the A (H3N2) winter influenza season in 2011-12, the correlations with the largest effect sizes were noted for log data from the website section "Influenza self-care and treatment" with no time lag to influenza-diagnosis cases $(r=0.94,95 \%$ CI $0.84-0.98 ; P<0.001)$ and "Influenza facts" with 1 week preceding lag $(r=0.94,95 \%$ CI $0.84-0.98 ; P<0.001)$.

\section{Correlations between GFT data, telenursing data, and log data from the county council website}

The correlations between GFT data and telenursing data showed large effect sizes for all epidemics (Table 5). During the first two winter influenza seasons in 2007-08 and 2008-09, the telenursing data preceded the GFT data by 1 week. However, during the A (pH1N1) pandemic in 2009 and the following two winter influenza seasons, the GFT data either preceded the telenursing 
data by 1 week or corresponded in time. The correlations between GFT data and log data from the county council website showed large effect sizes for the two epidemics for which data were available. For both the pandemic outbreak in $2009(r=0.87,95 \%$ CI $0.69-0.95 ; P<0.001)$ and the A (H3N2) winter influenza season in 2011-12 ( $r=0.96,95 \%$ CI $0.90-0.99$; $P<0.001)$, these two data streams showed optimal correlation when no time lag was introduced. Also, the correlations between telenursing data and log data from the county council website showed large effect sizes for the epidemics for which data were available. For the 2009 pandemic outbreak, the data streams showed optimal correlation when no time lag was introduced $(r=0.88,95 \%$ CI $0.71-$ $0.95 ; P<0.001)$, whereas during the winter influenza season in 2011-12, the optimal correlation was obtained when telenursing data preceded the log data from the county council website by 1 week $(r=0.95$, 95\% CI 0.84-0.99; $P<0.001)$.

Table 5. Associations on a weekly basis between GFT data and telenursing call data displayed by the correlation coefficient $r$ (95\% confidence interval) for the five influenza epidemics observed in Östergötland County, Sweden, during the study period 2007-12.

\begin{tabular}{|c|c|c|c|c|c|}
\hline $\begin{array}{l}\text { Epidemic } \\
\text { time lag } \\
\text { duration } \\
\text { (weeks) }\end{array}$ & $\begin{array}{l}\text { 2007-08 } \\
\text { (B and } A(H 1)) \\
(15 \text { weeks) }\end{array}$ & $\begin{array}{l}2008-09 \\
\text { (A (H3N2)) } \\
\text { (15 weeks) }\end{array}$ & $\begin{array}{l}2009 \\
\text { (A (pH1N1)) } \\
\text { (19 weeks) }\end{array}$ & $\begin{array}{l}\text { 2010-11 } \\
\text { (B and A } \\
\text { (pH1N1)) } \\
\text { (18 weeks) }\end{array}$ & $\begin{array}{l}2011-12 \\
\text { (A (H3N2)) } \\
\text { (14 weeks) }\end{array}$ \\
\hline-1 & $\begin{array}{l}0.88 \\
(0.65,0.96) \\
P=0.012\end{array}$ & $\begin{array}{l}0.92 \\
(0.77,0.98) \\
P=0.001\end{array}$ & n.s. & n.s. & $\begin{array}{l}0.90 \\
(0.69,0.97) \\
P=0.011\end{array}$ \\
\hline 0 & n.s. & $\begin{array}{l}0.88 \\
(0.68,0.96) \\
P=0.008\end{array}$ & $\begin{array}{l}0.77 \\
(0.49,0.91) \\
P=0.034\end{array}$ & $\begin{array}{l}0.85 \\
(0.63,0.94) \\
P=0.005\end{array}$ & $\begin{array}{l}0.94 \\
(0.83,0.98) \\
P=0.001\end{array}$ \\
\hline 1 & n.s. & n.s & $\begin{array}{l}0.87 \\
(0.68,0.95) \\
P=0.001\end{array}$ & $\begin{array}{l}0.94 \\
(0.83,0.98) \\
P<0.001\end{array}$ & $\begin{array}{l}0.87 \\
(0.60,0.96) \\
P=0.032\end{array}$ \\
\hline 2 & n.s. & n.s. & n.s. & $\begin{array}{l}0.81 \\
(0.53,0.93) \\
P=0.016\end{array}$ & $\begin{array}{l}0.86 \\
(0.56,0.96) \\
P=0.040\end{array}$ \\
\hline
\end{tabular}

Time -1 week $=$ Healthcare Direct/1177 1 week time shift, i.e., people first call Healthcare Direct/1177 and then use GFT 1 week later.

Time lag 1 week $=$ telenursing data 1 week time shift, i.e., people first use GFT and then call Healthcare Direct/1177 1 week later.

Time lag 2 weeks $=$ telenursing data 2 week time shift, i.e., people first use GFT and then call Healthcare Direct/1177 2 weeks later.

n.s., not statistically significant. 


\section{Preparatory phase: review and evaluation of existing detection and prediction algorithms}

\section{Studies fulfilling the inclusion criteria}

The search of the meta-narrative review identified eight studies reporting prospective algorithm performance based on data from naturally occurring influenza epidemics: three studies (Closas et al. 2012, Martínez-Beneito et al. 2008, Cowling et al. 2006) evaluated one or more detection algorithms and five (Timpka et al. 2014a, Yuan et al. 2013, Jiang et al. 2009, Burkom et al. 2007, Viboud et al. 2003) evaluated prediction algorithms (Figure 4).

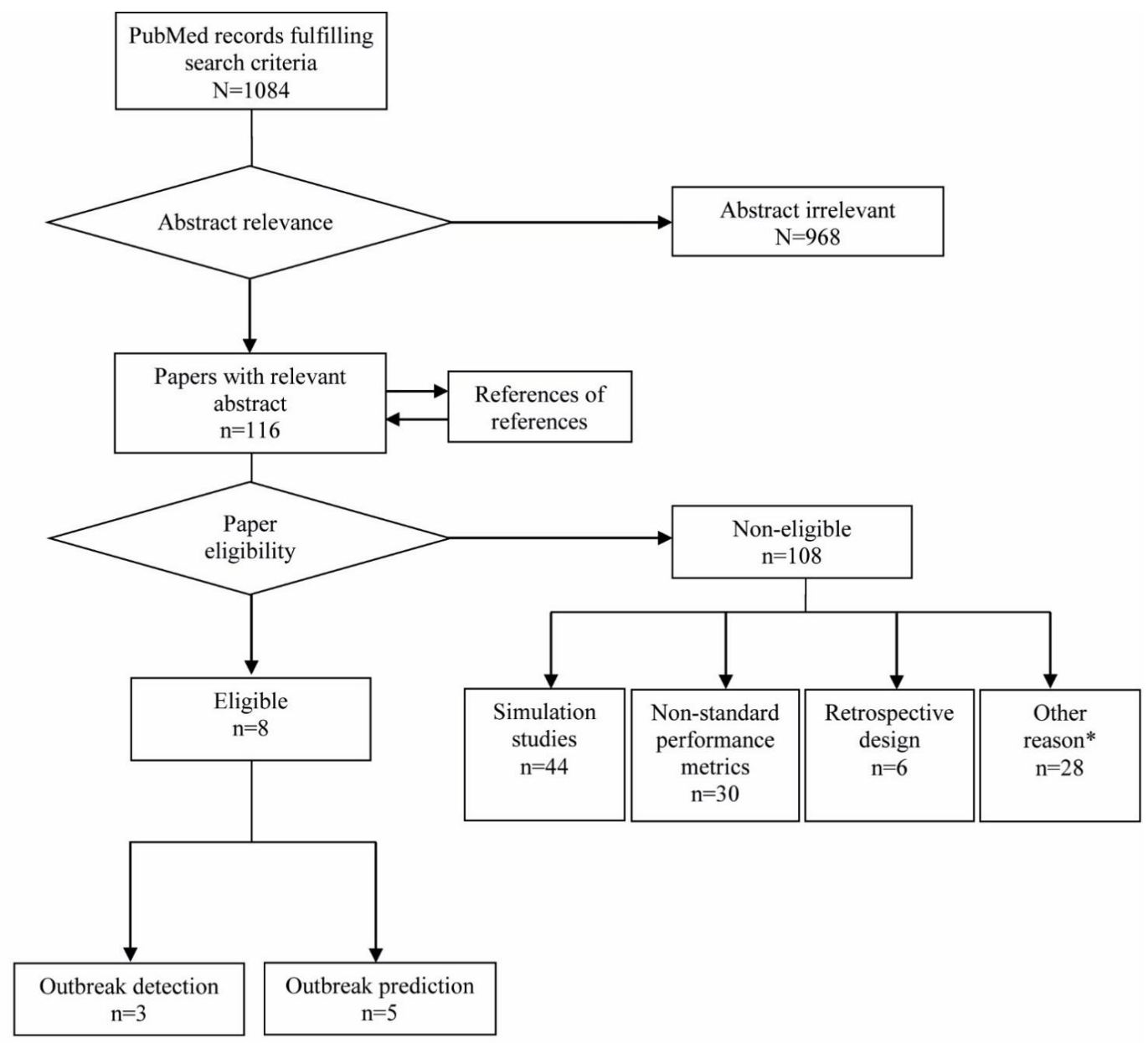

Figure 4. Flowchart of the paper selection process. Additional reasons for exclusion $(*)$ included that the case definition did not comprise at least a clinical diagnosis of influenza or influenza-like-illness. 


\section{Performance of the algorithms in their original settings}

Regarding influenza detection algorithms, outstanding performance was reported from a Spanish study (Martínez-Beneito et al. 2008) for Serfling regression along with two versions of algorithms based on hidden Markov models (Table 6). On the other hand, simple regression displayed poor performance in this study. However, the same method demonstrated excellent performance on influenza data from the United States in a study comparing algorithm performances on data from two continents, as did a time series method based on a dynamic model and the statistical process control method based on CUSUM (Cowling et al. 2006). When applied on data from Hong Kong in the same study, the performance of these three algorithms was found to be poor (for the CUSUM-based method) or acceptable (for simple regression and the time series method). 


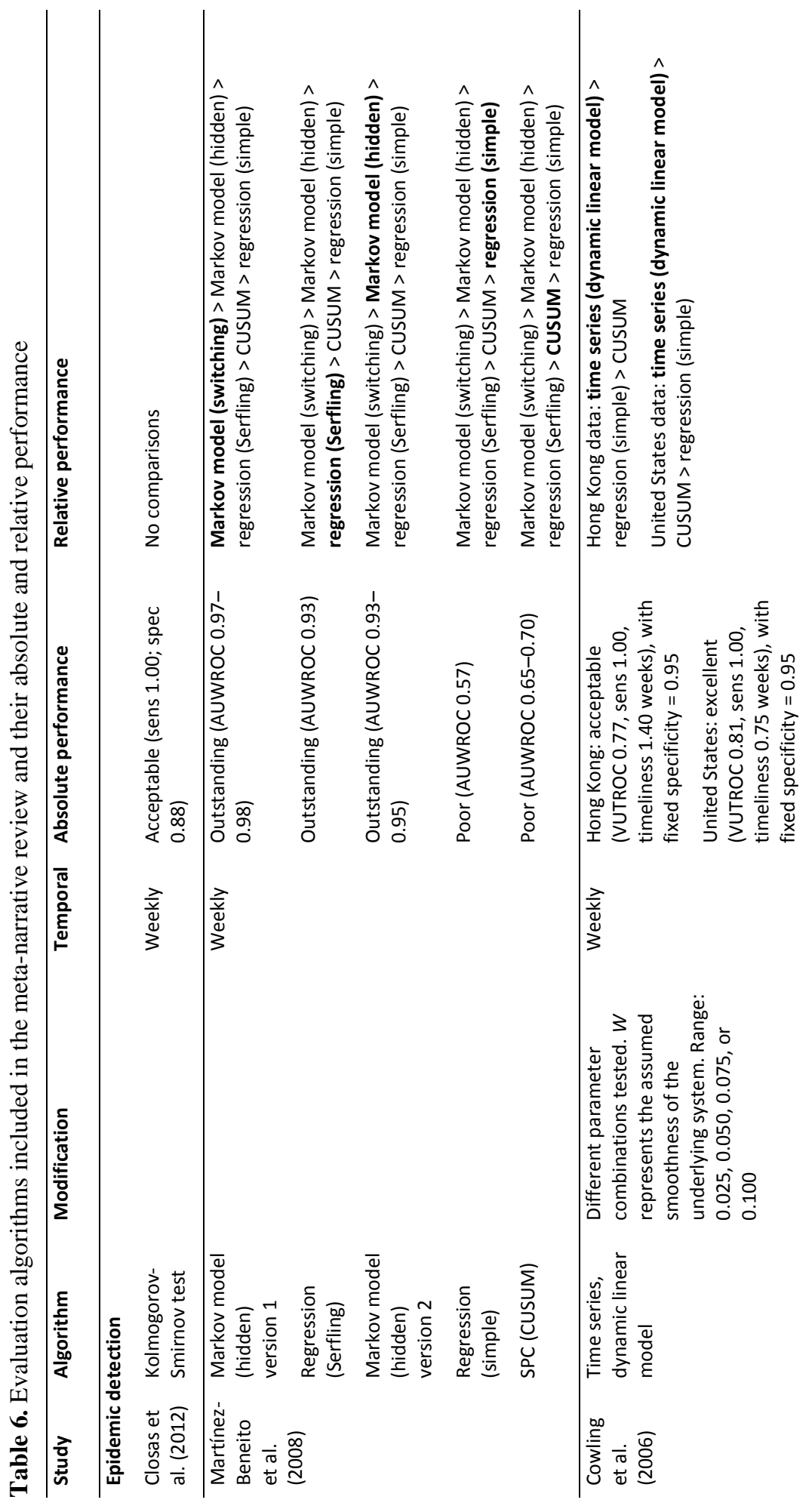




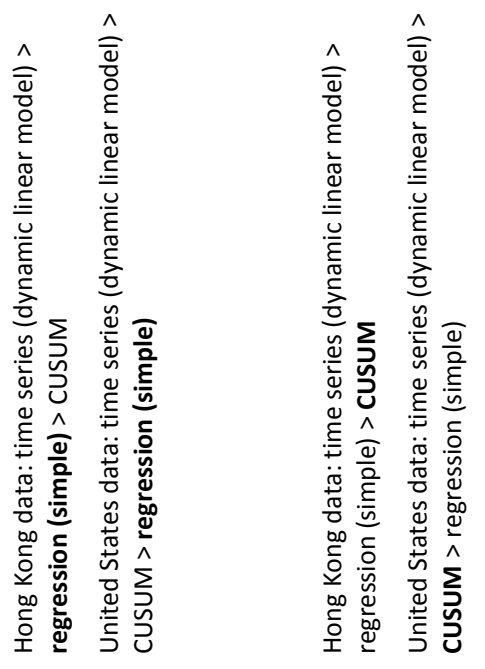

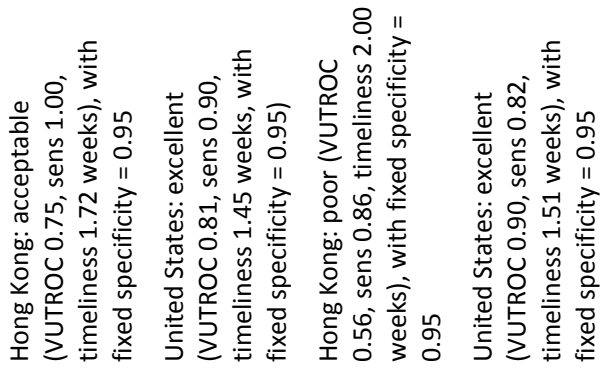

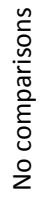

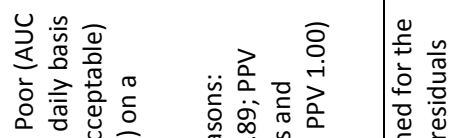

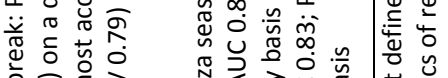

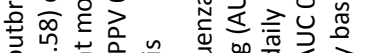

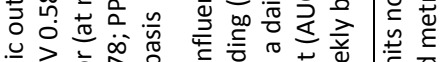

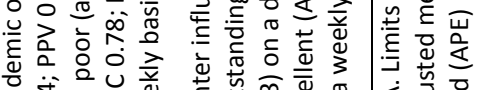

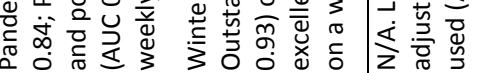

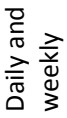




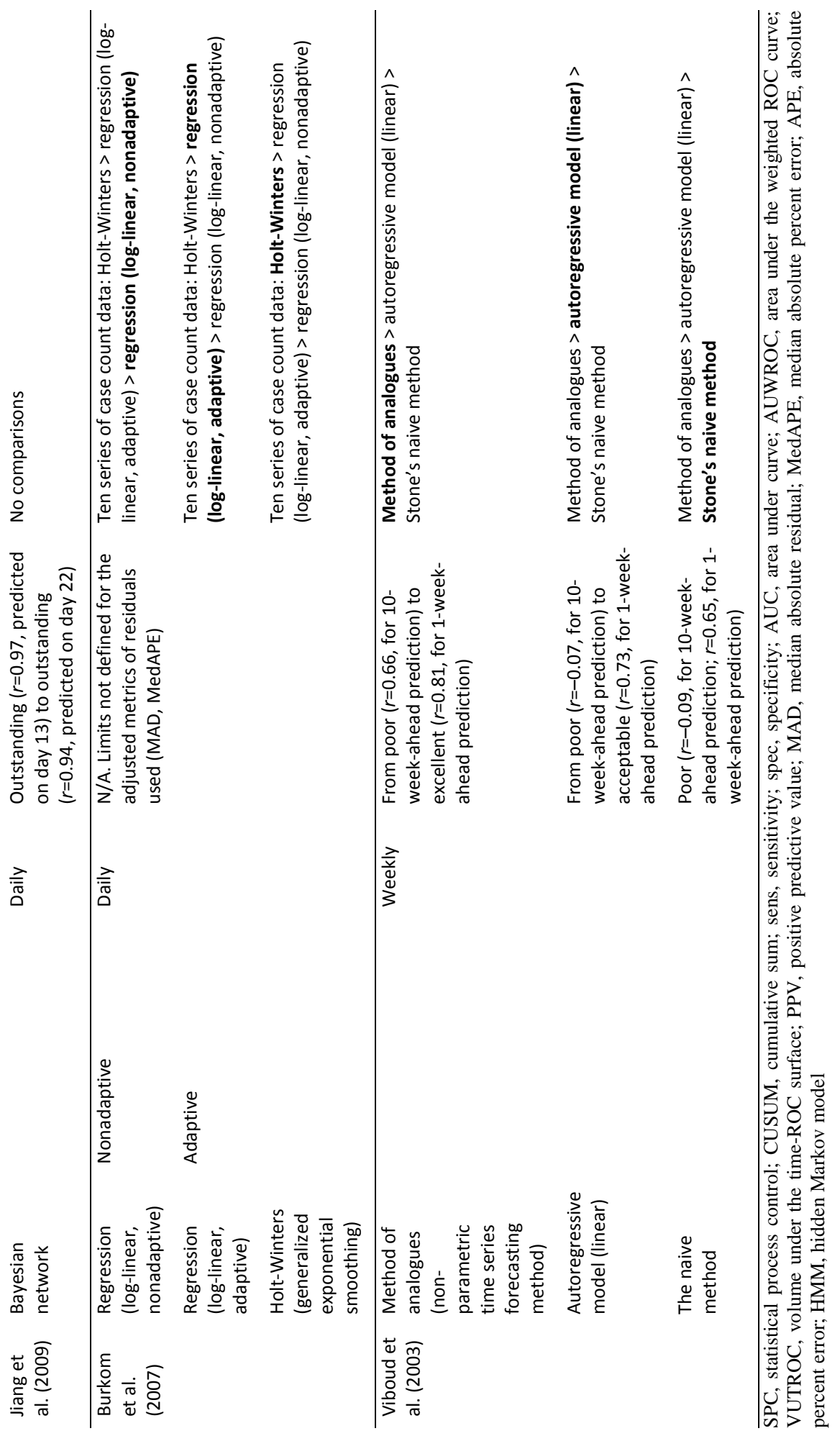


As regards influenza prediction algorithms, in a French study predicting national-level influenza epidemics over 18 seasons, excellent performance was observed for a non-parametric time series method in 1-week-ahead predictions, whereas the performance was poor in 10-week-ahead predictions (Viboud et al. 2003). Furthermore, outstanding predictive performance was displayed in a study applying a Bayesian network algorithm on county-level data from the United States (Jiang et al. 2009). However, in that study, the predictions were made on day 13 and day 22 of a single ongoing epidemic. Another study applying a Shewhart-type model on telenursing data from a Swedish county to predict influenza epidemics over three seasons, covering two winter influenza seasons and the A (pH1N1) pandemic in 2009, showed outstanding performance for the winter influenza seasons on a daily basis and excellent performance on a weekly basis (Timpka et al. 2014a). However, the performance was poor for the pandemic both on a daily basis and on a weekly basis.

Two additional studies evaluated four different prediction algorithms: multiple linear regression evaluated by Yuan et al. (2013) and log-linear nonadaptive regression, log-linear adaptive regression and the Holt-Winters method evaluated by Burkom et al. (2007). However, these studies used performance metrics that cannot be used for absolute performance comparison, only relative performance comparison, i.e., comparison of diverse methods applied to the same data.

\section{Narratives identified}

One explanation of the apparent variety of evaluation methods and findings is that the experiences and methodological perspectives from algorithm evaluations were reported in two distinct narrative formats. These narrative formats can be interpreted to represent biodefence informatics and health policy research, respectively (Table 7). 
Table 7. Summary of narrative characteristics.

\begin{tabular}{|c|c|c|c|c|c|c|}
\hline Narrative & Storyline & $\begin{array}{l}\text { Intended } \\
\text { audience }^{\mathrm{a}}\end{array}$ & $\begin{array}{l}\text { Learning } \\
\text { period } \\
\text { dilemma }\end{array}$ & $\begin{array}{l}\text { Theoretical } \\
\text { proofs }\end{array}$ & $\begin{array}{l}\text { Population } \\
\text { descriptions }\end{array}$ & $\begin{array}{l}\text { Endpoint } \\
\text { measures }\end{array}$ \\
\hline \multicolumn{7}{|l|}{$\begin{array}{l}\text { Biodefence } \\
\text { informatics }\end{array}$} \\
\hline $\begin{array}{l}\text { Closas et al. 2012, } \\
\text { Martínez-Beneito } \\
\text { et al. 2008, Jiang } \\
\text { et al. 2009, } \\
\text { Burkom et al. } \\
2007\end{array}$ & $\begin{array}{l}\text { System } \\
\text { verification }\end{array}$ & $\begin{array}{l}\text { Engineers } \\
\text { and } \\
\text { modelers }\end{array}$ & $\begin{array}{l}\text { Irregular } \\
\text { attention }\end{array}$ & $\begin{array}{l}\text { Included in } \\
\text { argument }\end{array}$ & Summary & $\begin{array}{l}\text { Various } \\
\text { statistical }\end{array}$ \\
\hline \multicolumn{7}{|l|}{$\begin{array}{l}\text { Health } \\
\text { policy research }\end{array}$} \\
\hline $\begin{array}{l}\text { Cowling et al. } \\
\text { 2006, Timpka et } \\
\text { al. 2014a, Yuan et } \\
\text { al. } 2013 \text {, Viboud } \\
\text { et al. } 2003\end{array}$ & $\begin{array}{l}\text { System } \\
\text { validation }\end{array}$ & $\begin{array}{l}\text { Policy } \\
\text { makers }\end{array}$ & $\begin{array}{l}\text { Binding } \\
\text { attention }\end{array}$ & Excluded & Extensive & $\begin{array}{l}\text { Standard } \\
\text { epidemiological }\end{array}$ \\
\hline
\end{tabular}

a. In addition to the researchers.

\section{The biodefence informatics narrative}

Assessments informing the construction of mathematically and technically sound algorithms for infectious disease detection and prediction have been reported from mathematical modeling and health informatics contexts. Research in these fields was described in a biodefence informatics narrative. The setting for this narrative is formative evaluation and justification of algorithms for detection and prediction of atypical epidemics of infectious diseases and bioterror attacks. In other words, studies in this narrative can be considered to answer the system verification question "Did we build the system right?" (Boehm 1981). The narrative is set in a context where it is essential to modify and ensure the algorithms for detection and prediction of microbiological agents with rare or unknown features, e.g., novel influenza virus strains or anthrax (Gursky \& Bice 2012). After the attacks on the World Trade Center in 2001, the number of studies presented in the biodefence informatics narrative increased rapidly (Paterson \& Durrheim 2013). Demonstration of technical or statistical developments characterizes the reporting of influenza algorithm performance in this narrative, with, for instance, the use of increments instead of rates or the introduction of methods based on Markov models (Martínez-Beneito et al. 2008). In this narrative, limited attention is paid to the learning period dilemma, because, for logical reasons, empirical data are restricted in biodefence settings. A general methodological challenge in algorithm development is the learning period 
dilemma, i.e., algorithm parameters are determined using observations from one time interval (the learning period) and then used to predict the occurrence of corresponding events in a later interval (the evaluation period) (Altman \& Royston 2000). When aiming to detect or predict a novel infectious disease, the unavailability of learning data for calibration of model-based algorithms

primarily characterizes the learning period dilemma. For example, for prediction algorithms relying on the reproductive number (White et al. 2009), a sufficiently long series of learning data for empirical determination of the serial interval is not available during the early stages of an epidemic, which implies that the method cannot be used as supposed (Klick et al. 2014). Furthermore, both environmental conditions and microbiological features of the pathogen during the learning period can change after the algorithm components and parameters have been defined, calling for adjustments to be made to preserve the predictive performance. A combination of prospective evaluations with formal proofs and analyses of retrospective and simulated data in the biodefence informatics narrative can be used to verify the performance of an algorithm. The evaluation results are included in the narrative, although it is commonly emphasized that they are preliminary with regard to population outcomes (Jiang et al. 2009).

\section{The health policy research narrative}

Studies evaluating algorithms of this kind should follow the general criteria established for health policy evidence in order for the study results to qualify as input to recommendations regarding infectious disease control practice. The analyses cannot be open to manipulation and must be unbiased; for example, the data sources and analytical models should be fixed and described before data are accessed for analyses (Kelly et al. 2010). Moreover, the use of prospective study designs is regarded as the cornerstone in the research process in the corresponding research paradigm (Derose et al. 2002). Studies reported in the health policy research narrative can be considered to answer the validation question "Have we built the right system for detection and prediction of influenza epidemics?". Even though data on clinical diagnoses and data from laboratory tests were mainly used in the studies reported in this narrative, the two most recently reported studies also used syndromic data. One of these used data from telenursing call centers (Timpka et al. 2014a), while the other used data from an Internet search engine (Yuan et al. 2013). The basis in real-world validation of alerts and predictions was shown in this narrative, for instance, by pointing out that most often only a small amount of annual 
infectious disease cycles of data are accessible for evaluations of new algorithms, leading to a constant shortage of evidence-based information on which to base policy (Cowling et al. 2006). This basis was also demonstrated by the fact that space was provided for discussions concerning whether changes in epidemic conditions would yield less accurate performances, e.g., that the intensity levels during pandemics are higher than the ones noted during interepidemic periods (Timpka et al. 2014a, Viboud et al. 2003). Furthermore, the evaluations included in this narrative emphasize the quantitative strength of the research evidence. For example, the evaluation period lasted 938 weeks and covered an entire nation in the French study evaluating a non-parametric time series method (Viboud et al. 2003). On the other hand, a study in the biodefence informatics narrative evaluated a Bayesian network model (Jiang et al. 2009) in one US county using a 26-week-long evaluation period.

\section{Algorithms considered for the comparative accuracy trial}

\section{Description of the detection algorithms evaluated}

At the time of algorithm selection, seven influenza detection algorithms were found to have been evaluated using authentic prospective data. Three of these algorithms met the criteria of the comparative accuracy trial: Serfling regression (Martínez-Beneito et al. 2008, Serfling 1963), simple regression (Cowling et al. 2006, Stroup et al. 1989), and CUSUM (Cowling et al. 2006, Martínez-Beneito et al. 2008). These three algorithms, together with two modified versions of the CUSUM algorithm, were evaluated in the comparative trial.

Serfling regression (Martínez-Beneito et al. 2008, Serfling 1963) monitors the period when there is no increased influenza activity to determine a baseline defined by a fixed threshold. Defining $\widehat{Y}_{t}$ as the number of events (in this particular case the number of influenza-diagnosis cases or telenursing calls), the model is expressed as

$\widehat{Y}_{t}=b_{0}+b_{1} t+b_{2(t)} \cos (k t)+b_{3(t)} \sin (k t)$,

where $b_{0}$ is a constant intercept, $b_{1}$ is the slope of a long-term trend and $b_{2(t)}$ and $b_{3(t)}$ are coefficients for continuous harmonic terms representing seasonal trends, with $k=2 \pi /(365.25 / 7)$ to give a 1-year sinusoidal period of these terms. Using only the non-epidemic phases of the pre-defined learning set, first the regression coefficients above were determined. Applying these to the learning set, the optimal threshold $\alpha$ (the threshold that generates the best 
combination of sensitivity and specificity), which is based on the normal distribution, was searched for by investigating $\alpha=0.005,0.010, \ldots, 0.500$.

Simple regression (Cowling et al. 2006, Stroup et al. 1989) raises an alarm if data from the current week fall outside a $100(1-\alpha) \%$ forecast interval from a normal distribution with running mean $\tilde{y}_{(\mathrm{m})}$ and running sample variance $\tilde{s}^{2}(\mathrm{~m})$ calculated from the preceding $m$ weeks. The forecast interval is calculated as $\tilde{y}_{(m)} \pm t_{m-1,1-\alpha / 2} \tilde{S}_{(m)} \sqrt{1+\frac{1}{m}}$, where $t_{m-1,1-\alpha / 2}$ is the $100(1-\alpha)$ th percentile of the Student $t$ distribution with $m-1$ degrees of freedom. Using the learning set, the parameter combination resulting in the highest sensitivity and specificity for this algorithm was searched for, investigating all possible combinations of $\alpha=$ $0.005,0.010, \ldots, 0.500$ and $m=3,4, \ldots, 10$.

The CUSUM method (Cowling et al. 2006, Martínez-Beneito et al. 2008) raises an alarm if the upper CUSUM $C_{t}^{+}$exceeds a pre-specified threshold $g$. For the series of observations $y_{t}, t=1,2, \ldots, t$, the $d$-week upper CUSUM at time $t, C_{t}^{+}$is defined as

$C_{t}^{+}=\max \left(0, \frac{y_{t}-\tilde{y}_{(7)}}{\tilde{s}_{(7)}}-k+C_{t-1}^{+}\right)$,

with $C_{t-d}^{+}=0$ (Montgomery 2005). The running mean $\tilde{y}_{(7)}$ and running variance $\tilde{s}^{2}(7)$ are calculated from the series of 7 weeks, $y_{i-d-7}, \ldots ., y_{i-d-1}$, preceding the most recent $d$ weeks. $d$ denotes the number of weeks excluded from the running mean and variance immediately before the index week. This exclusion is done to avoid contamination with the upswing of an epidemic (Tokars et al. 2009). The parameter $k$ represents the minimum standardized difference from the running mean, which is not ignored by the CUSUM calculation.

The CUSUM algorithm was, as mentioned, evaluated in its original form and in two modified versions based on the fact that the observations $y$ follow a Poisson distribution. In the first modified version, the variance is estimated by the sample mean because the variance equals the expected value in a Poisson distribution. In the second modified version, CUSUM at time $t\left(C_{t}\right)$ is expressed in terms of accumulated probability, namely $C_{t}=\max \left(0 ; C_{t-1}+\right.$ $\left.P\left(Y \leq y \mid E=\tilde{y}_{(7)}\right)-k\right)$, where $P$ is the Poisson probability function. The second suggested modification is an adaptation to Poisson distributed data with such low expected values that normal approximation is inappropriate. In the second modified version, the pre-specified alarm threshold $(g)$ cannot be based on the 
normal distribution. Using the learning set, the parameter combination generating the highest sensitivity and specificity for all three version of the CUSUM method was sought, investigating all possible combinations of $g=$ $0.00,0.01, \ldots, 20.00, k=0.00,0.01, \ldots, 3.00(k=0.00-1.00$ for the third version) and $d=0,1, \ldots, 4$ for influenza-diagnosis data; and $g=0.00,0.01, \ldots, 100.00$, $k=0.00,0.01, \ldots, 4.00 \quad(k=0.00-1.00$ for the third version $)$ and $d=$ $0,1, \ldots, 4$ for telenursing data.

\section{Description of the prediction algorithms evaluated}

In the meta-narrative review, nine influenza prediction algorithms were found to have been evaluated using authentic prospective data. However, only three of these met the secondary inclusion criteria of the comparative trial: nonadaptive log-linear regression, adaptive log-linear regression (Burkom et al. 2007), and the naive method (Viboud et al. 2003). Consequently, these three were the only prediction algorithms evaluated in this study.

The first algorithm evaluated, nonadaptive log-linear regression, fits an ordinary least squares, log-linear model to a pre-defined learning set to obtain regression coefficients (Brillman et al. 2005). These coefficients are then used to forecast values beyond the learning data without adjusting for subsequent changes in time series behavior. Modified for weekly data, the model is expressed as

$\log \left(Y_{t}+1\right)=b_{0}+b_{1} t+b_{2} \cos (k t)+b_{3} \sin (k t)$,

where $Y_{t}$ is the number of influenza-diagnosis cases or telenursing calls on week $t, b_{0}$ is a constant intercept, $b_{1}$ is the slope of a long-term trend, and $b_{2}$ and $b_{3}$ are coefficients for continuous harmonic terms representing seasonal trends, with $k=2 \pi /(365.25 / 7)$ to give a 1-year sinusoidal period of these terms. The original weekly counts were transformed into log scale to capture the multiplicative nature of the effects of the trend and seasonal components.

The second algorithm evaluated, adaptive log-linear regression with a sliding 8 -week baseline interval, recomputes the regression coefficients for each forecast using only the series values from the 8 weeks before the forecast week (Burkom 2003). The short baseline is intended to capture recent seasonal and trend patterns (Burkom et al. 2007). Modified for weekly data, the model is described as

$\log \left(Y_{t}+1\right)=b_{0}+b_{1} t$ 
where $Y_{t}$ is the number of influenza-diagnosis cases or telenursing calls on week $t, b_{0}$ is a constant intercept, and $b_{1}$ is the slope of a long-term trend. In Burkom et al. (2007), a holiday indicator was added to avoid exaggerated holidays occurring in the short baseline interval; however, because weekly counts were used in this comparative trial, the holiday effect was considered to be low or non-existent. Adjustments to the suggested models were made to fit weekly counts because Burkom et al. (2007) used daily counts in their study.

The final algorithm being evaluated in this study, the so-called naive method, predicts that a future incidence $F$ is equal to a current incidence $I$, hence $F(T+h)=I(T)$, where $h \geq 1$ and $T$ is the current week number.

\section{Detection and prediction algorithms excluded}

Four of the detection algorithms did not meet the secondary inclusion criteria of the comparative accuracy trial. The algorithm based on the KolmogorovSmirnov test evaluated by Closas et al. (2012) was excluded because it was not applicable on streams of county-level influenza-diagnosis data. This test assumes that the rate of influenza-diagnoses in non-epidemic periods can be represented by a random variable $(y)$ that is exponentially distributed. However, influenza-diagnosis data in local settings are in general represented by small integers during non-epidemic periods. Therefore, it is more reasonable to assume that the observations are Poisson distributed. The time series method based on a dynamic model (Cowling et al. 2006) was excluded for similar reasons, i.e., it requires that data follow a normal distribution. Finally, the two hidden Markov models evaluated by Martínez-Beneito et al. (2008) were excluded because the algorithms partly relies on simulated data.

Six of the prediction algorithms did not meet the secondary inclusion criteria of the comparative accuracy trial. The Holt-Winters method (generalized exponential smoothing; Burkom et al. 2007) and the method of analogues (Viboud et al. 2003) were excluded because they required collection of learning data from more than one winter influenza season. The autoregressive model (Viboud et al. 2003) was excluded because the evaluation data used in this study did not comply with its detailed assumptions. A Bayesian network model (Jiang et al. 2009), a Shewhart-type algorithm (Timpka et al. 2014a), and a multiple linear regression algorithm (Yuan et al. 2013) were excluded because they required access to multidimensional data, i.e., a syndromic data source to predict the number of influenza-diagnosis cases. 


\section{Retrospective algorithm calibration}

When re-applied on the retrospective influenza-diagnosis data, four of the detection algorithms evaluated in the comparative accuracy trial (Serfling regression and the three CUSUM methods) demonstrated perfect performance (i.e., specificity and sensitivity 1.00 and timeliness 0 week) (Table 8 ). Regarding syndromic telenursing data, the grouping of telenursing chief complaints with the largest correlation strength on a weekly basis $(r=0.91 ; P<0.001)$ and longest lead time ( 2 weeks) to influenza-diagnosis cases in the retrospective data was fever (child, adult). Following these findings, fever (child, adult) was used as the complaint grouping in the evaluations. The detection algorithms with the best performance when re-applied on retrospective telenursing data were Serfling regression and the three CUSUM methods (specificity 1.00, sensitivity 0.80 , and timeliness -2 weeks) (Table 8). On the other hand, all four showed less accurate performance than when applied to influenza-diagnosis data. The simple regression model underperformed when re-applied both to diagnostic data (specificity 0.90, sensitivity 0.70, and timeliness 23 weeks) and to telenursing data (specificity 0.90 , sensitivity 0.50 , and timeliness 12 weeks).

Table 8. Performance of influenza detection algorithms when applied retrospectively on the learning set of influenza-diagnosis data and syndromic telenursing data.

\begin{tabular}{lllll}
\hline Algorithm & $\begin{array}{l}\text { Parameter } \\
\text { combination }\end{array}$ & Specificity & Sensitivity & Timeliness $^{\mathrm{a}}$ \\
\hline $\begin{array}{l}\text { Influenza-diagnosis data } \\
\text { Serfling regression }\end{array}$ & $\alpha=0.035$ & 1.00 & 1.00 & 0 \\
Simple regression & $m=9$ weeks, $\alpha=0.075$ & 0.90 & 0.70 & $23^{\mathrm{b}}$ \\
CUSUM ver1 & $d=2, k=0.25, g=18.49$ & 1.00 & 1.00 & 0 \\
CUSUM ver2 & $d=2, k=0.25, g=20.73$ & 1.00 & 1.00 & 0 \\
CUSUM ver3 & $d=2, k=0.60, g=4.55$ & 1.00 & 1.00 & 0 \\
Telenursing data & & & & \\
Serfling regression & $\alpha=0.135$ & 1.00 & 0.80 & -2 \\
Simple regression & $m=9$ weeks, $\alpha=0.075$ & 1.00 & 0.50 & 12 \\
CUSUM ver1 & $d=2, k=0.13, g=15.16$ & 1.00 & 0.80 & -2 \\
CUSUM ver2 & $d=2, k=0.13, g=25.07$ & 1.00 & 0.80 & -2 \\
CUSUM ver3 & $d=2, k=0.55, g=3.93$ & 1.00 & 0.80 & -2 \\
\hline
\end{tabular}

a. Positive timeliness means that the alarm is raised before the epidemic has started (i.e., the alarm is raised too early) and negative timeliness means that the alarm is raised after the epidemic has started (i.e., the alarm is raised too late).

b. One value stands out, otherwise the timelines would have been 0 .

The prediction algorithm performing best when re-applied to the retrospective influenza-diagnosis dataset was the nonadaptive log-linear regression $(r=0.72$ 
for 2-weeks-ahead predictions and $r=0.57$ for 4-weeks-ahead predictions) followed by the naive method ( $r=0.62$ for 2 -weeks-ahead predictions and $r=0.29$ for 4-weeks-ahead predictions) (Table 9). On the other hand, nonadaptive loglinear regression had the least accurate MedAPE among the methods evaluated (0.92 for 2-week predictions and 1.00 for 4-week predictions). For syndromic telenursing data, the nonadaptive log-linear regression was superior $(r=0.72$ for 2-week predictions to $r=0.66$ for 4 -week predictions and MedAPE 0.15 for 2week predictions and 0.16 for 4 -week predictions) to the other two algorithms evaluated (Table 9).

Table 9. Performance of influenza prediction algorithms when applied retrospectively on the learning set of influenza-diagnosis data and syndromic telenursing data.

\begin{tabular}{|c|c|c|c|c|}
\hline & \multirow[b]{2}{*}{ Algorithm } & \multicolumn{3}{|c|}{ Prediction $k$ weeks ahead } \\
\hline & & 2 weeks & 3 weeks & 4 weeks \\
\hline \multicolumn{5}{|c|}{ Influenza-diagnosis data } \\
\hline \multirow[t]{3}{*}{ Correlation $(r)^{\text {a }}$} & Nonadaptive log-linear regression & 0.72 & 0.65 & 0.57 \\
\hline & Adaptive log-linear regression ${ }^{\mathrm{b}}$ & 0.47 & 0.26 & 0.12 \\
\hline & The naive method ${ }^{c}$ & 0.62 & 0.45 & 0.29 \\
\hline \multirow[t]{3}{*}{ MedAPE } & Nonadaptive log-linear regression & 0.92 & 1.00 & 1.00 \\
\hline & Adaptive log-linear regression ${ }^{\mathrm{b}}$ & 0.76 & 0.86 & 0.98 \\
\hline & The naive method ${ }^{c}$ & 0.74 & 0.77 & 1.00 \\
\hline \multicolumn{5}{|l|}{ Telenursing data } \\
\hline \multirow[t]{3}{*}{ Correlation $(r)^{\mathrm{a}}$} & Nonadaptive log-linear regression & 0.72 & 0.69 & 0.66 \\
\hline & Adaptive log-linear regression ${ }^{b}$ & 0.45 & 0.27 & 0.14 \\
\hline & The naive method ${ }^{c}$ & 0.64 & 0.49 & 0.34 \\
\hline \multirow[t]{3}{*}{ MedAPE ${ }^{a}$} & Nonadaptive log-linear regression & 0.15 & 0.16 & 0.16 \\
\hline & Adaptive log-linear regression ${ }^{\mathrm{b}}$ & 0.28 & 0.34 & 0.40 \\
\hline & The naive method ${ }^{c}$ & 0.14 & 0.20 & 0.20 \\
\hline
\end{tabular}

a. The correlation coefficient ( $r$ ) ranks the algorithms from highest (best) to lowest (worst) values, whereas MedAPE ranks them from lowest (best) to highest (worst) values.

b. The method does not have the same learning set because it is adaptive, which means that the parameters are updated every week.

c. The method has no learning set. The predicted value $k$ weeks ahead is the same as the true value $k$ weeks before.

\section{Prospective evaluations of detection algorithms}

In the prospective evaluation using influenza-diagnosis data, the best performing detection algorithm during the winter influenza seasons was Serfling regression (specificity 1.00, sensitivity 0.80 , and timeliness -2 weeks), whereas the CUSUM Poisson (version 2) was the best performing algorithm during the pandemic outbreak in 2009 (specificity 0.80, sensitivity 1.00, and timeliness 1 week) (Table 10). In the corresponding evaluation using syndromic 
telenursing data, Serfling regression displayed the best performance both during winter influenza seasons (specificity 1.00 , sensitivity 0.55 , and timeliness -4 weeks) and during the pandemic outbreak (specificity 1.00 , sensitivity 0.70 , and timeliness -2 weeks) (Table 10). However, the performance was evidently unsatisfactory for all of the detection algorithms (including Serfling regression) when applied to telenursing data.

Table 10. Performance of influenza detection algorithms when applied prospectively on influenza-diagnosis data and syndromic telenursing data.

\begin{tabular}{|c|c|c|c|c|c|c|c|}
\hline \multirow[b]{2}{*}{ Algorithm } & \multirow[b]{2}{*}{$\begin{array}{l}\text { Parameter } \\
\text { combination }\end{array}$} & \multicolumn{3}{|c|}{ Winter influenza seasons } & \multicolumn{3}{|c|}{ Pandemic } \\
\hline & & $\begin{array}{l}\text { Spec- } \\
\text { ificity }\end{array}$ & $\begin{array}{l}\text { Sens- } \\
\text { itivity }\end{array}$ & $\begin{array}{l}\text { Time- } \\
\text { liness }^{\mathrm{a}}\end{array}$ & $\begin{array}{l}\text { Spec- } \\
\text { ificity }\end{array}$ & $\begin{array}{l}\text { Sens- } \\
\text { itivity }\end{array}$ & $\begin{array}{l}\text { Time- } \\
\text { liness }^{a}\end{array}$ \\
\hline \multicolumn{8}{|c|}{ Influenza-diagnosis data } \\
\hline Serfling regression & $\alpha=0.035$ & 1.00 & 0.80 & -2 & 0.60 & 0.90 & 4 \\
\hline Simple regression & $m=9$ weeks, $\alpha=0.075$ & 0.70 & 0.75 & 20 & 0.57 & 0.30 & 4 \\
\hline CUSUM ver1 & $d=2, k=0.25, g=18.49$ & 0.65 & 1.00 & 3 & 0.60 & 1.00 & 2 \\
\hline CUSUM ver2 & $d=2, k=0.25, g=20.73$ & 0.65 & 0.95 & $4^{b}$ & 0.80 & 1.00 & 1 \\
\hline CUSUM ver3 & $d=2, k=0.60, g=4.55$ & 0.85 & 0.70 & $5^{b}$ & 1.00 & 0 & $--^{c}$ \\
\hline \multicolumn{8}{|l|}{ Telenursing data } \\
\hline Serfling regression & $\alpha=0.135$ & 1.00 & 0.55 & -4 & 1.00 & 0.70 & -2 \\
\hline Simple regression & $m=9$ weeks, $\alpha=0.075$ & 0.80 & 0.45 & 19 & 0.86 & 0.30 & 5 \\
\hline CUSUM ver1 & $d=2, k=0.13, g=15.16$ & 0.20 & 1.00 & 9 & 1.00 & 0.50 & -5 \\
\hline CUSUM ver2 & $d=2, k=0.13, g=25.07$ & 0.25 & 0.95 & 7 & 1.00 & 0.40 & -6 \\
\hline CUSUM ver3 & $d=2, k=0.55, g=3.93$ & 0.40 & 0.80 & 7 & 1.00 & 0 & $-^{c}$ \\
\hline
\end{tabular}

a. Positive timeliness means that the alarm is raised before the epidemic has started (i.e., the alarm is raised too early) and negative timeliness means that the alarm is raised after the epidemic has started (i.e., the alarm is raised too late).

b. The mean of the absolute values.

c. An alarm is never raised.

\section{Prospective evaluations of prediction algorithms}

During the winter influenza seasons, the prediction algorithm displaying the best predictive performance when applied to influenza-diagnosis data was onadaptive log-linear regression ( $r=0.77$ for 2-weeks-ahead predictions, $r=0.76$ for 4-weeks-ahead predictions, and MedAPE varying from 0.75 for 2-week predictions to 0.77 for 4 -week predictions); none of the algorithms provided satisfactory evaluation outcomes during the pandemic outbreak (Table 11). Using syndromic telenursing data, nonadaptive log-linear regression generated the best predictive performance during the winter influenza seasons $(r=0.77$ for 2-week predictions, $r=0.69$ for 4 -week predictions, and MedAPE varying from 0.17 for 2-weeks-ahead predictions to 0.20 for 4 -weeks-ahead predictions) (Table 11), whereas all three algorithms evaluated produced substantially 
lower correlations during the pandemic outbreak; the highest correlation was $r=0.52$ for the naive method for 2-weeks-ahead predictions.

Table 11. Performance of influenza prediction algorithms when applied prospectively on influenza-diagnosis data and syndromic telenursing data.

\begin{tabular}{|c|c|c|c|c|c|c|c|}
\hline & \multirow[b]{3}{*}{ Algorithm } & \multicolumn{6}{|c|}{ Prediction $k$ weeks ahead } \\
\hline & & \multicolumn{3}{|c|}{ Winter influenza seasons } & \multicolumn{3}{|c|}{ Pandemic } \\
\hline & & 2 weeks & 3 weeks & 4 weeks & 2 weeks & 3 weeks & 4 weeks \\
\hline \multicolumn{8}{|c|}{ Influenza-diagnosis data } \\
\hline \multirow[t]{3}{*}{ Correlation $(r)^{\mathrm{a}}$} & Nonadaptive log-linear regression & 0.77 & 0.77 & 0.76 & -0.27 & -0.30 & -0.33 \\
\hline & Adaptive log-linear regression ${ }^{\mathrm{b}}$ & 0.52 & 0.35 & 0.22 & 0.09 & 0.01 & 0.02 \\
\hline & The naive method ${ }^{c}$ & 0.62 & 0.44 & 0.28 & 0.38 & 0.18 & 0.07 \\
\hline \multirow[t]{3}{*}{ MedAPE ${ }^{a}$} & Nonadaptive log-linear regression & 0.75 & 0.77 & 0.77 & 1.00 & 0.97 & 0.97 \\
\hline & Adaptive log-linear regression ${ }^{\mathrm{b}}$ & 1.00 & 1.00 & 1.00 & 0.96 & 1.00 & 1.21 \\
\hline & The naive method ${ }^{c}$ & 0.80 & 0.90 & 0.98 & 0.70 & 0.77 & 0.81 \\
\hline \multicolumn{8}{|l|}{ Telenursing data } \\
\hline \multirow[t]{3}{*}{ Correlation $(r)^{\mathrm{a}}$} & Nonadaptive log-linear regression & 0.77 & 0.73 & 0.69 & -0.20 & -0.27 & -0.34 \\
\hline & Adaptive log-linear regression ${ }^{\mathrm{b}}$ & 0.67 & 0.55 & 0.42 & 0.37 & 0.24 & 0.18 \\
\hline & The naive method ${ }^{c}$ & 0.74 & 0.64 & 0.51 & 0.52 & 0.37 & 0.22 \\
\hline \multirow[t]{3}{*}{ MedAPE ${ }^{a}$} & Nonadaptive log-linear regression & 0.17 & 0.19 & 0.20 & 0.31 & 0.29 & 0.28 \\
\hline & Adaptive log-linear regression ${ }^{\mathrm{b}}$ & 0.19 & 0.23 & 0.33 & 0.34 & 0.37 & 0.39 \\
\hline & The naive method ${ }^{c}$ & 0.17 & 0.19 & 0.23 & 0.19 & 0.21 & 0.26 \\
\hline
\end{tabular}

a. The correlation coefficient ( $r$ ) ranks the algorithms from highest (best) to lowest (worst) values, whereas MedAPE ranks them from lowest (best) to highest (worst) values.

b. The method does not have the same learning set because it is adaptive, which means that the parameters are updated every week.

c. The method has no learning set. The predicted value $k$ weeks ahead is the same as the true value $k$ weeks before.

\section{Development phase: design and evaluation of the nowcasting method}

\section{Method design overview}

Following the main aim of this thesis, an influenza nowcasting method was developed. The method is based on non-mechanistic statistical models, i.e., trends and patterns in the data are modeled without necessarily considering underlying mechanisms, and is designed for integration in local health information systems. Accordingly, the underpinning structure is defined at four levels, ranging from data sources to performance validation (Figure 5). The method is divided into separate modules for the detection and prediction of influenza activity. The purpose of the detection module is to alert local health care services to a forthcoming period of increased load of influenza-diagnosis cases, whereas the purpose of the prediction module is to predict the peak timing and its intensity. Streams of gold standard data are used both for early 
detection of increased influenza activity and for peak intensity prediction, and syndromic data are used for peak timing prediction. In this study, patients clinically diagnosed with influenza were used as the gold standard, whereas telenursing data were used as the syndromic data source.

An overview of the main statistical assumptions and equations for each component is displayed in Figure 6, followed by detailed descriptions of these. 


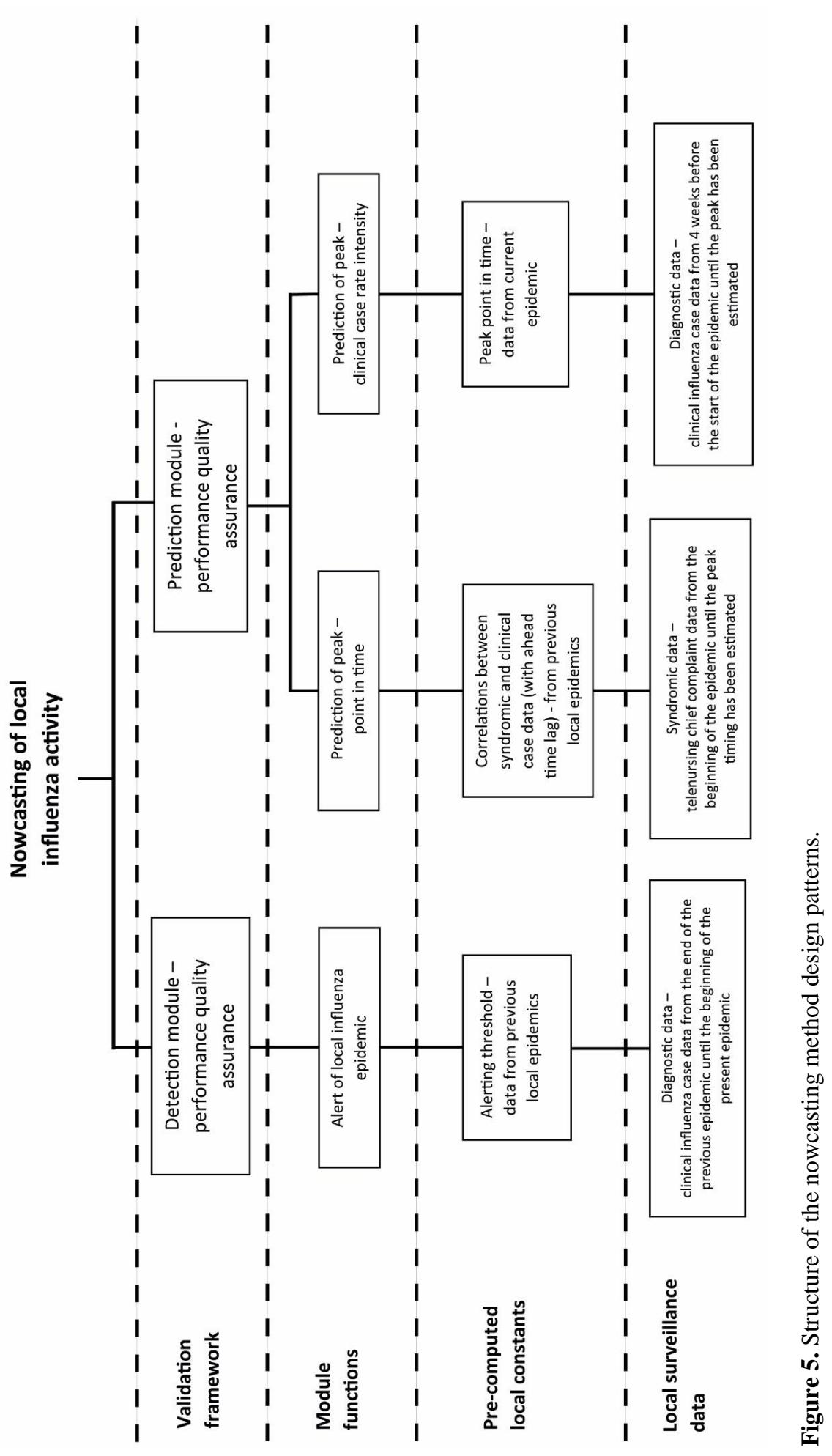




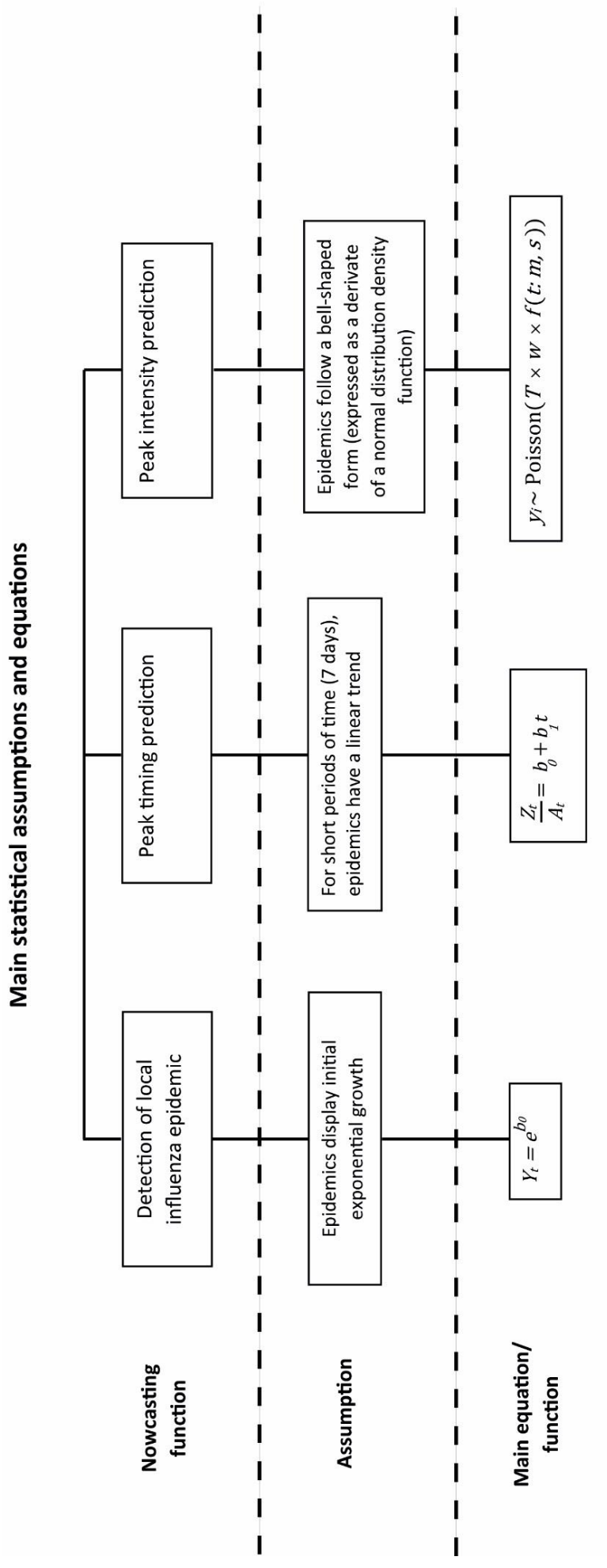

เก

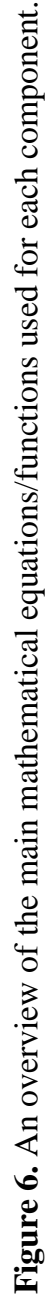




\section{Detection module}

Exponential regression (1) is used for detection modeling, based on the observation that the beginning of an influenza epidemic is assumed to have exponential growth of the number of infected individuals:

$$
\text { (1) } X_{t}=e^{a_{0}+b_{1} t} \text {, }
$$

with $t$ representing the time, ao representing the level, and $b_{1}$ representing the trend. The expected number of visits to local health care services, $E\left[Y_{t}\right]$ is the product of $X$ and the probability $p$ of an infected individual visiting the local health care service. This expectation is also exponential in time:

$$
\text { (2) } E\left[Y_{t}\right]=e^{a_{0}+b_{1} t} p=e^{a_{0}+\ln p+b_{1} t}=e^{b_{0}+b_{1} t} \text {, }
$$

where $b_{0}$ now combines the current level of the number of infected individuals and the probability of visiting the local health care service without any possibility of separating them. Because daily data are used in the analysis, weekday effects, $A_{w}$, are also calculated and used as an offset variable in the exponential regression analysis. The weekday effects are calculated as follows: let $A_{\text {Monday }}$ be the average number of events on Mondays during previous epidemics and denote the values for other weekdays by $A_{\text {Tuesday, }} A_{\text {wednesday }}$ etc. Let $A_{\text {Total }}=\left(A_{\text {Monday }}+\ldots+A_{\text {sunday }}\right) / 7$. The multiplicative weekday effect for Mondays is $A_{\text {Monday }} / A_{\text {Total }}$ etc. The weekday effects are included in the model:

(3) $E\left[Y_{t}\right]=e^{b_{0}+b_{1} t+\ln A_{w}}$,

If $X$ is large, $p$ is small and the infected individuals act independently, then $Y$ is approximately Poisson distributed;

(4) $Y_{t} \sim \operatorname{Poisson}\left(e^{b_{0}+b_{1} t+\ln A_{w}}\right)$.

Furthermore, the time is shifted, i.e., the most recent day is considered as $t=0$, the second most recent day is considered as $t=-1$ etc. For every new day, the time axis is moved one step so that the new "most recent day" is considered as $t=0$. For each day, an exponential regression analysis (1) is run and a fitted value, $\hat{y}$, is calculated by inserting $t=0$ in equation (3) giving

(5) $Y_{t}=e^{b_{0}}$,

as an estimate of the current level of visits, which is smoothed for random variation and adjusted for weekday effects. This is repeated for each day by moving the time axis one day at a time so that the most recent point in time of the series is considered $t=0$. By doing this, one value is obtained for every day representing the level for that day. Finally, the lower 95\% confidence limit is calculated to represent the level of influenza activity, which then is compared with a predetermined threshold. If the level is above the threshold, an alarm is raised meaning that the epidemic has started, and if the level is below the threshold, no alarm is raised. 
Detection starts when the previous epidemic has ended (the inter-epidemic period level for the community where the detection component is applied), and runs during the inter-epidemic period until an increase in influenza-diagnosis cases is detected. When the increase is confirmed the algorithm is paused and restarted when the epidemic has ended.

The detection algorithm is adjusted in exceptional situations, i.e., if an epidemic "simmers" before it begins. The risk of simmering is extensive for a pandemic or an exceptionally mild winter influenza season. In the first case, if there is fear of a pandemic outbreak among the population, individuals are more likely to contact medical services for influenza symptoms leading to an increased baseline, which increases the risk for false alarms. Also, if a winter influenza season is exceptionally mild, individuals contacting medical services for influenza-like symptoms in the winter will sporadically be misdiagnosed with influenza before the actual circulation of the influenza virus, leading to an increased baseline and thus an increased risk for false alarms. The alerting threshold determined in the learning set is therefore doubled in these particular situations. It was contended that a strong indication of pre-epidemic simmering is when it takes an extended time between the influenza incidence increasing above a baseline level and the start of the epidemic (according to the standard definition of 6.3 influenza-diagnosis cases/100,000 over a floating 7-day period). The definition for when the influenza incidence has increased above the baseline level is set to 3.2 influenza-diagnosis cases/100,000 over a floating 7day period (i.e., half that of the definition for the start of an epidemic). An epidemic is then defined as simmering if the time period separating these two dates is longer than three times the average length of the period during previous local influenza epidemics. In other words, the alerting threshold is only doubled as a result of simmering if the incidence has increased over the baseline level, but not exceeded the start-of-epidemic level during this observation period. Concerning pandemics, a local influenza epidemic was defined to be part of a pandemic based on a corresponding WHO alert.

Two exceptional situations were identified during the evaluation period: one pandemic outbreak and one mild winter influenza season. Accordingly, the alarm threshold was doubled after the pandemic alert from the WHO (2009), and before the simmering winter influenza season in 2013-14. 


\section{Prediction module}

The prediction process is divided into two components. In the first component, syndromic data are used to predict the peak timing and in the second component, influenza-diagnosis data are used to estimate the peak intensity.

\section{Peak timing prediction}

In the first component of the prediction module, the aim is to predict the peak timing using linear regression. Including weekday effects $A_{w}$ and smoothed for random variation, the model for the number of cases in syndromic data is expressed as

(6) $Z_{t}=\left(b_{0}+b_{1} t\right) * A_{w}$

with $b_{0}$ representing the level and $b_{1}$ representing the trend. Because the weekday effects $A_{w}$ are known, a model smoothed for weekday effects and random variation can be expressed as

$$
\text { (7) } \frac{z_{t}}{A_{w}}=b_{0}+b_{1} t
$$

For each 7-day period, a linear regression (7) is run and parameter estimates $b_{0}$ and $b_{1}$ are fitted. The idea is to estimate the trend in syndromic data for every 7 -day period (the first period being days $1-7$, the second days 2-8), from the beginning of an epidemic and until the peak is found. Although it is unlikely that an epidemic curve increases and decreases linearly, the assumption can be made that the trend during a short period of 7 days has almost a linear increase or decrease.

The search for the peak starts when the detection algorithm signals that an epidemic has begun and continues until the peak is detected. To identify the peak timing, two conditions are set. According to the first condition, it is essential to ensure that the epidemic has a sufficiently sharp upward trend. The trend is therefore defined as sufficiently sharp when significantly positive $(P<0.05)$ trends $b_{1}$ have occurred either during two consecutive or during three different 7-day periods. When one of these circumstances has occurred, the second condition is applied. According to this condition, when the first significantly negative trend $b_{1}$ during a 7-day period has occurred, it is assumed that the peak has been reached on the first day of this period. However, there is a possibility that this 7-day period "overlaps" with a previous 7-day period that includes a significantly positive trend. In that case, the first 7-day period with a significantly negative trend is ignored and the peak is instead assumed to appear during the second 7-day period with a significantly negative trend. The search is aborted if the peak is not found when 
the epidemic has already descended in the local setting where the algorithm is applied.

When the peak is found in the syndromic data, the 14 days preceding the influenza-diagnosis data (Timpka et al. 2014b) is utilized to find the influenzadiagnosis peak. In other words, if the peak in the syndromic data appears on day 0 , the influenza-diagnosis peak is assumed to appear on day 14 . However, it is possible that the peak in the syndromic data occurs on a day during the weekend but highly unlikely that the peak in influenza-diagnosis data occurs on one of these days, because primary care centers are closed during weekends in Sweden. Instead, it is reasonable to assume that the influenza-diagnosis peak occurs at the beginning of the week because individuals who have influenza symptoms during the weekend visit primary care centers when they re-open on Monday or possibly Tuesday. Adjustments are therefore made by moving the influenza-diagnosis peak to the following Monday if it is expected to occur on a Friday, Saturday or Sunday according to the syndromic data, and to the previous Tuesday if the peak is expected to take place on a Wednesday or Thursday. If the peak is expected to occur on a Monday or Tuesday, no adjustments are made. In other words, in the first case, the syndromic data precede influenza-diagnosis data by between 15 and 17 days, in the second case by between 12 and 13 days, and in the third case by 14 days.

Depending on what day of the week the peak in the syndromic data is expected to take place, the prediction of the influenza-diagnosis peak is made between 6 and 11 days before it is expected to occur, because the syndromic peak can be determined first after 6 days of the syndromic data series has passed.

\section{Peak intensity prediction}

In the second component of the prediction module, the aim is to predict only the peak intensity. Based on empirical assessments of previous epidemics, an epidemic adjusted for weekday effects is assumed to show a bell-shaped form from the beginning to the end, and can therefore be expressed using a derivate of a normal distribution density function. The intensity function must also include weekday effects and the total number of influenza-diagnosis cases during the whole epidemic. The use of bell-shaped functions was systematically introduced in epidemiology by Brownlee in the early 20th century (Brownlee 1915), and since then, such functions have been applied in several contexts, e.g., to predict the course of AIDS in the United States (Bregman \& Langmuir 1990). Assuming that the peak timing is known (estimated in the first prediction component) and that an epidemic follows a 
bell-shaped function around the peak, the intensity function can be used to predict the peak intensity at time $m$. Assume that on day number $t=1,2,3, \ldots$, $t_{i}$, the observed number of influenza-diagnosis cases is $y=y_{1}, y_{2}, y_{3}, \ldots, y_{i}$ and that

$$
\text { (8) } y_{i} \sim \operatorname{Poisson}(T \times w \times f(t: m, s)) \text {, }
$$

where $T$ is the total number of health care visits for the whole epidemic, $w$ is the weekday effect, $f$ is the normal distribution density function, $t$ is the day number, $m$ is the center of the epidemic (which coincides with $t$ for the peak), and $s$ is the spread in time. Because $t, w$, and $m$ are known, only the parameters $T$ and $s$ are estimated using $y$ in such way that the likelihood is maximized. However, in order to do that, appropriate starting values for these parameters need to be selected. Finally, using the known parameter $m$ and the estimated parameters $T$ and $s$, the peak intensity at time $m$ is calculate by replacing $t$ with $m$ in equation (8).

Also, it is important that the start of the series seems appropriate because the peak intensity prediction component assumes that the level is zero or at an inter-epidemic level at the start and it is not optimal if there are single/occasional spikes at the beginning of the series. For that reason, the start of the series should be a couple of weeks before an epidemic is detected.

\section{Retrospective performance of the nowcasting method}

The learning period included the 2008-09 winter influenza season.

\section{Detection module}

The optimal threshold for the lower confidence limit of the expected number of influenza-diagnosis cases in the retrospective learning dataset was calculated to be $0.21 /$ day $/ 100,000$ for the detection algorithm. The detection timeliness was 0 days and the sensitivity and specificity were both 1.00 (Figure 7). In other words, the nowcasting method detected the 2008-09 winter influenza season on its starting day according to the definition (6.3 influenza-diagnosis cases/100,000 over a floating 7-day period). 


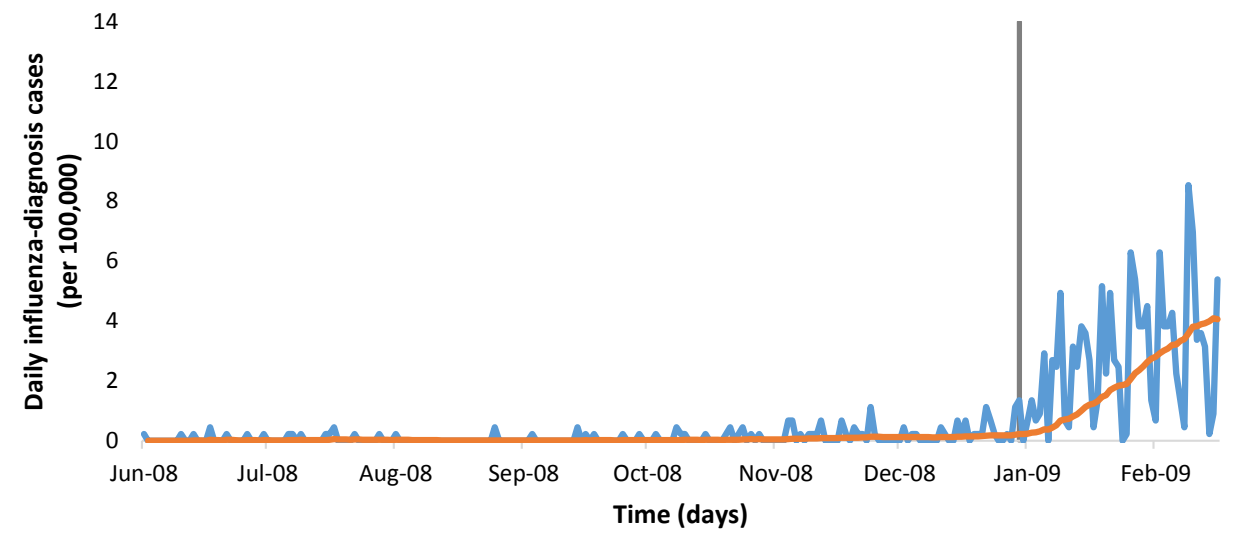

Figure 7: The detection algorithm applied to winter influenza season 2008-09 (A (H3N2)). The blue line represents the number of influenza-diagnosis cases/day/100,000, the gray bar marks the start of the winter influenza season according to the definition (6.3 influenzadiagnosis cases/100,000 over a floating 7-day period), and the orange line denotes the lower limit estimated using the detection algorithm.

\section{Prediction module}

The performance of the prediction module was satisfactory both with regard to the peak timing and its intensity. The peak timing was estimated 8 days in advance and occurred 7 days before the actual peak occurred. The predicted peak intensity at the predicted peak day was estimated to 6.3 influenzadiagnosis cases/100,000 (Figure 8), compared with the actual number of 8.5 influenza-diagnosis cases $/ 100,000$ on the day of the actual peak, i.e., the absolute difference between the predicted and the actual incidence was $26 \%$. 


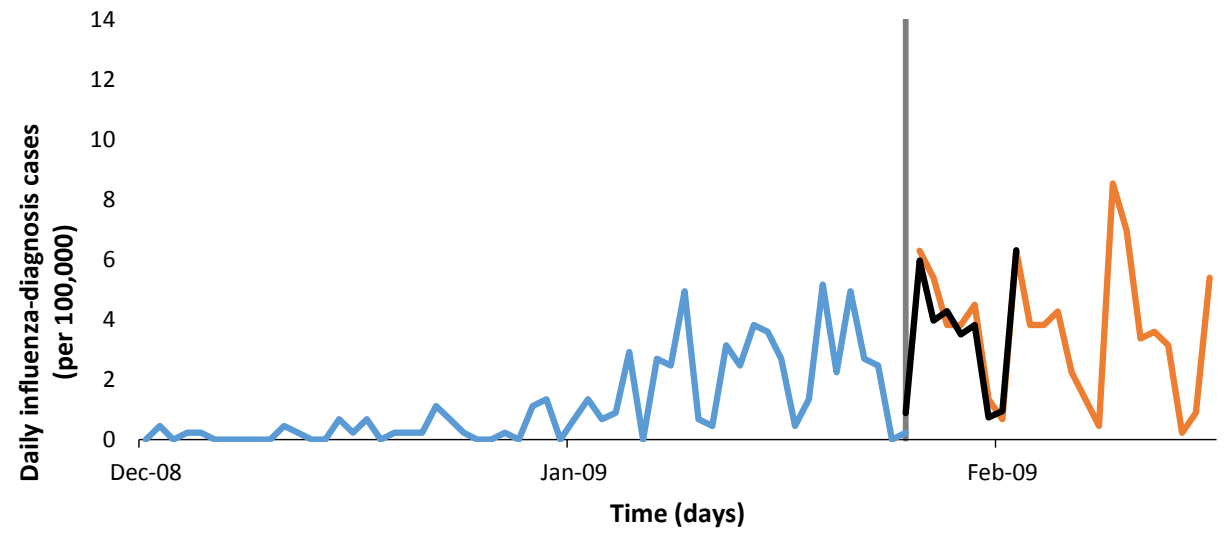

Figure 8. The prediction method applied to the winter influenza season 2008-09 (A (H3N2)). The blue line represents the number of known actual influenza-diagnosis cases/day/100,000 at the time the prediction was made, the orange line represents the number of "unknown" actual influenza-diagnosis cases/day/100,000 at the time when the prediction was made from the first unknown day and until the peak has passed, the gray bar marks the end of the known and the beginning of the "unknown" actual influenza-diagnosis cases/day/100,000, and the black line denotes the predicted values (using the peak intensity prediction) from the first "unknown" day until the predicted peak occurs.

\section{Prospective evaluation of the nowcasting method}

The evaluation period included one pandemic (2009) and four winter influenza seasons in 2010-11, 2011-12, 2012-13, and 2013-14 (Figure 9). 
14
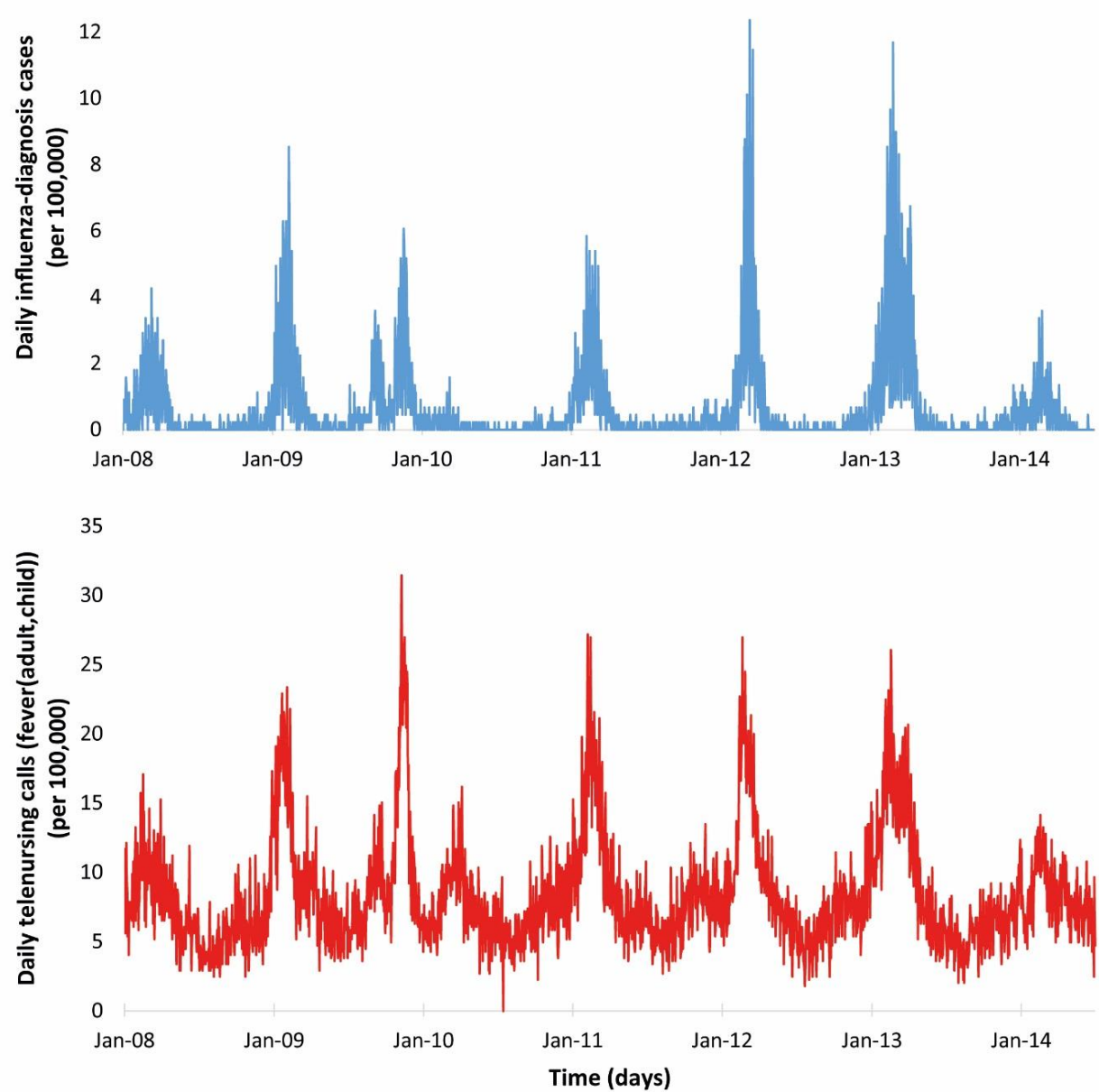

Figure 9. Unadjusted daily numbers of influenza-diagnosis cases (upper graph) and telenursing calls due to fever (adult, child) (lower graph) per 100,000 population from 1 January 2008 to 30 June 2014 in Östergötland County, Sweden.

\section{Local detection}

Good performance was found for the detection module of the local nowcasting method during the 2009 influenza A (pH1N1) pandemic, detecting the increase in local influenza activity 5 days after the pandemic outbreak actually started (Table 12, Figure 10). Also during the following winter influenza season in 201011 with influenza B and influenza A (pH1N1) circulating, the local detection performance was good with the alarm raised 5 days after the actual start of the season (Figure 11). However, the performance of the detection module was poor during the next winter influenza season (in 2011-12) with only influenza 
A (H3N2) circulating; the alarm was raised 15 days before the actual start of the season (Figure 12). For the last two winter influenza seasons evaluated, 2012-13 and 2013-14, the detection performance was excellent with alarms raised 3 days before (Figure 13) and 3 days after (Figure 14) the season started, respectively.

Table 12. Performance of the detection algorithm displayed with alerting thresholds updated using data from previous winter influenza seasons.

\begin{tabular}{llll}
\hline Influenza activity & $\begin{array}{l}\text { Updated threshold } \\
\text { (cases/day/100,000) }\end{array}$ & Timeliness $^{\mathrm{a}}$ & Interpretation \\
\hline 2009 (pandemic) A (pH1N1) & 0.424 & -5 & Good \\
\hline 2010-11 B and A (pH1N1) & 0.212 & -5 & Good \\
2011-12 A (H3N2) & 0.207 & 15 & Poor \\
2012-13 A (H3N2), B and A (pH1N1) & 0.242 & 3 & Excellent \\
2013-14 A (H3N2), B and A (pH1N1) & 0.481 & -3 & Excellent \\
\hline
\end{tabular}

a. A positive value means that the algorithm issued an alarm before the local epidemic started and a negative value means the alarm was raised after the start of the epidemic.

b. The threshold was doubled because of a pandemic alert or observation of a period of simmering influenza activity.

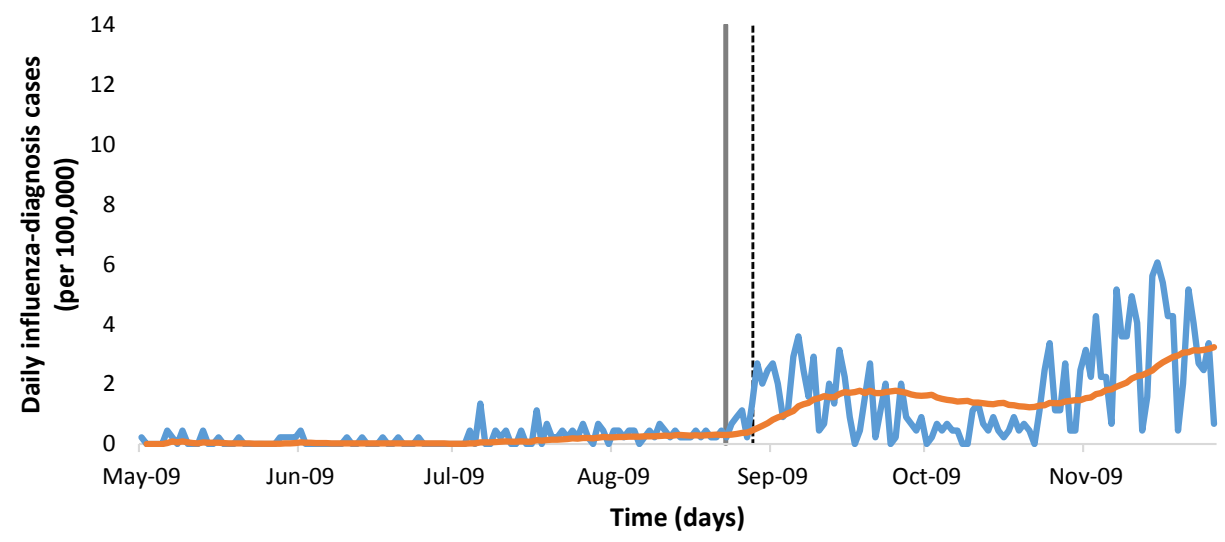

Figure 10. The detection algorithm prospectively applied to the 2009 pandemic outbreak (A (pH1N1)). The blue line represents the number of influenza-diagnosis cases/day/100,000, the dotted bar marks the start of the pandemic outbreak according to the algorithm, the gray solid bar marks the start of the outbreak according to the definition (6.3 influenza-diagnosis cases/100,000 over a floating 7-day period), and the orange line denotes the lower limit estimated using the detection algorithm. 


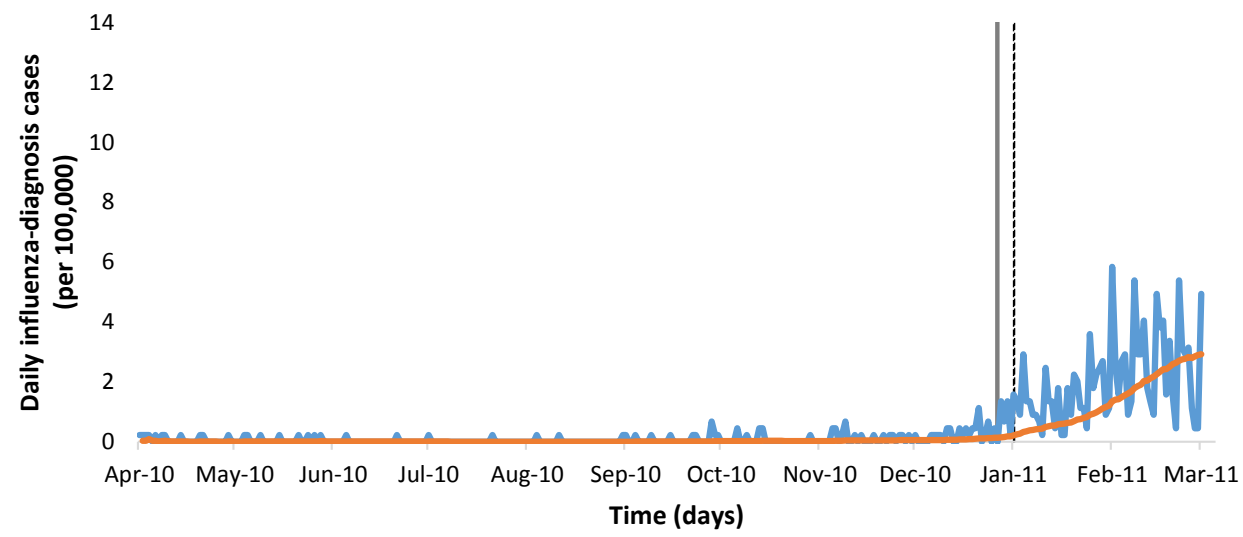

Figure 11. The detection algorithm applied prospectively to winter influenza season 2010-11 (B and $\mathrm{A}(\mathrm{pH} 1 \mathrm{~N} 1))$. The blue line represents the number of influenza-diagnosis cases/day/100,000, the dotted bar marks the start of the influenza season according to the algorithm, the gray solid bar marks the start of the influenza season according to the definition (6.3 influenza-diagnosis cases/100,000 over a floating 7-day period) and the orange line denotes the lower limit estimated using the detection algorithm.

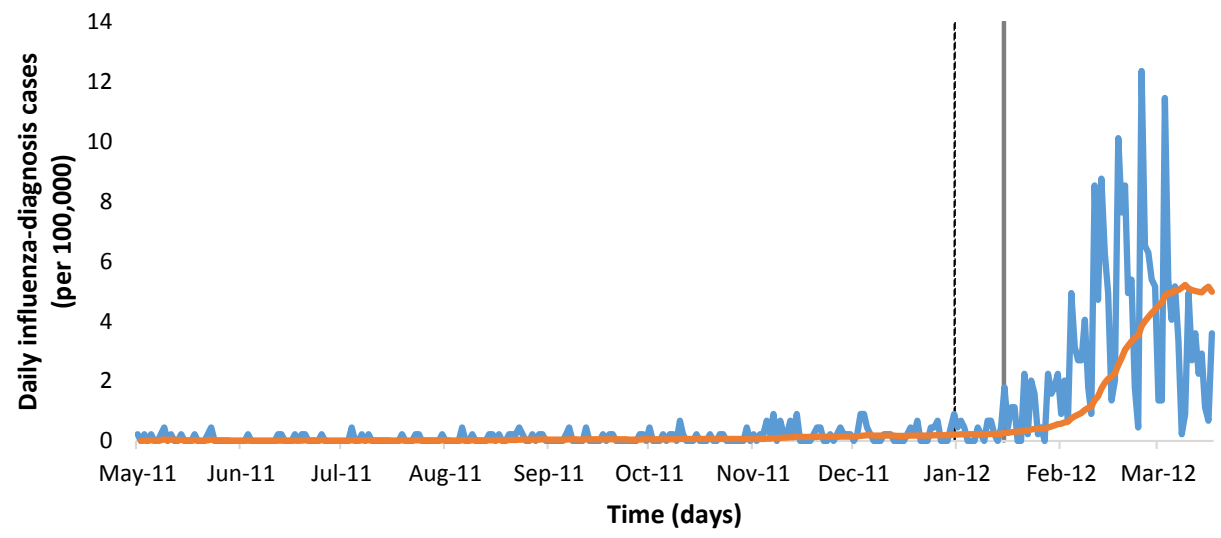

Figure 12. The detection algorithm applied prospectively to winter influenza season 2011-12 (A (H3N2)). The blue line represents the number of influenza-diagnosis cases/day/100,000, the dotted bar marks the start of the influenza season according to the algorithm, the gray solid bar marks the start of the influenza season according to the definition (6.3 influenzadiagnosis cases/100,000 over a floating 7-day period), and the orange line denotes the lower limit estimated using the detection algorithm. 


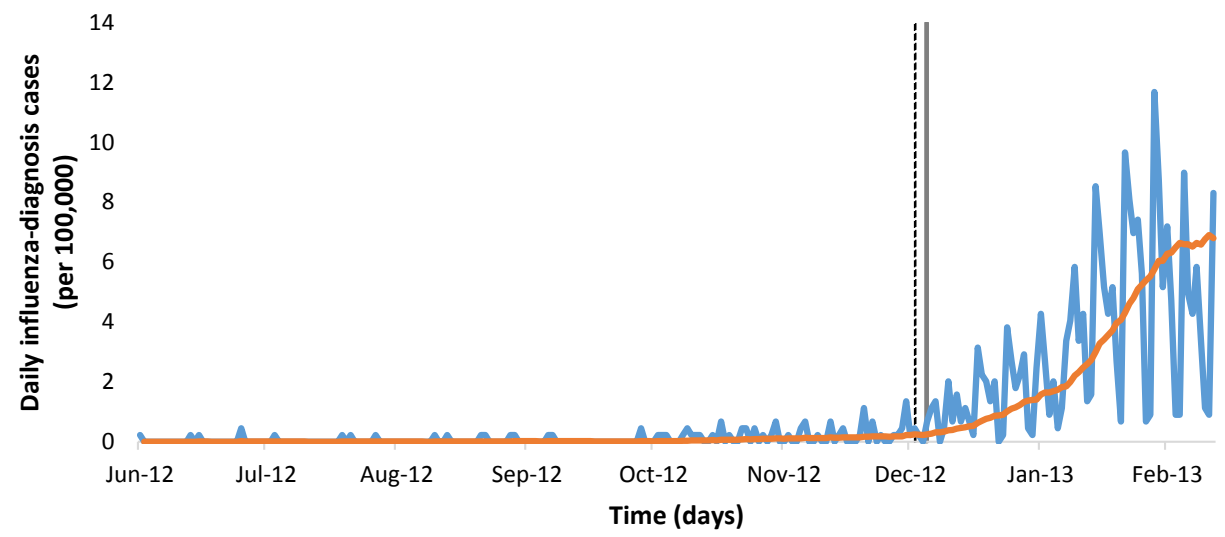

Figure 13. The detection algorithm applied prospectively to winter influenza season 2012-13 (A (H3N2), B and A (pH1N1)). The blue line represents the number of influenza-diagnosis cases/day/100,000, the dotted bar marks the start of the influenza season according to the algorithm, the gray solid bar marks the start of the influenza season according to the definition (6.3 influenza-diagnosis cases/100,000 over a floating 7-day period), and the orange line denotes the lower limit estimated using the detection algorithm.

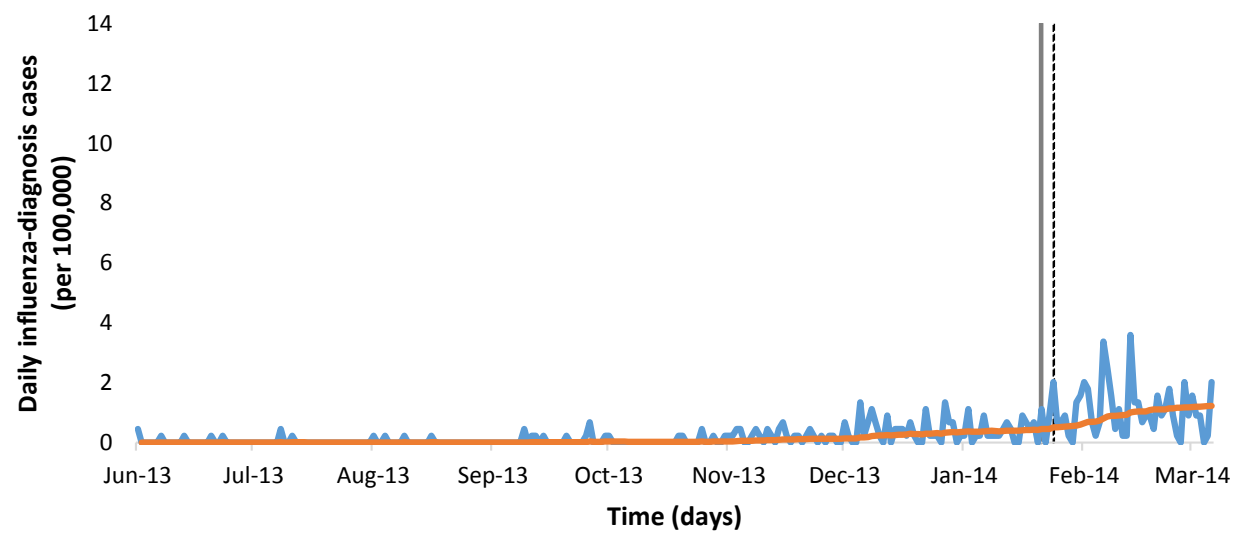

Figure 14. The detection algorithm applied prospectively to winter influenza season 2013-14 (A (H3N2), B and A (pH1N1)). The blue line represents the number of influenza-diagnosis cases/day/100,000, the dotted bar marks the start of the influenza season according to the algorithm, the gray solid bar marks the start of the influenza season according to the definition (6.3 influenza-diagnosis cases/100,000 over a floating 7-day period), and the orange line denotes the lower limit estimated using the detection algorithm.

\section{Local prediction}

During the 2009 influenza A (pH1N1) pandemic, the performance of the local peak timing prediction was poor, whereas the peak intensity level was successfully predicted to the correct category (medium intensity epidemic) (Table 13, Figure 15). For the following winter influenza season of 2010-11 with 
influenza B and influenza A (pH1N1) circulating, the local peak timing prediction was excellent and the peak intensity level of the epidemic was correctly categorized as a medium intensity epidemic (Figure 16). Furthermore, the local peak timing predictions were good for the next two winter influenza seasons (i.e., 2011-12 and 2012-13) with influenza A (H3N2) circulating during the first of these seasons and influenza A (H3N2), influenza B, and influenza A ( $\mathrm{pH} 1 \mathrm{N1}$ ) circulating during the second season. The local peak intensity predictions were unsuccessful for the 2011-12 winter influenza season categorizing it wrongly as a medium intensity epidemic when it in fact developed into a very high intensity epidemic (Figure 17), and successful for the 2012-13 winter influenza season predicting it as the correct category (very high intensity epidemic) (Figure 18). During the 2013-14 winter influenza season with influenza B and influenza A ( $\mathrm{pH} 1 \mathrm{~N} 1)$ circulating and a simmering start, the performance of the local peak timing prediction was acceptable, whereas the local peak intensity was wrongly categorized as a non-epidemic level when the season actually reached a medium intensity level (Figure 19).

Table 13. Performance of peak timing and peak intensity predictions.

\begin{tabular}{|c|c|c|c|c|c|c|c|}
\hline \multirow[t]{2}{*}{ Influenza activity } & \multicolumn{4}{|c|}{$\begin{array}{l}\text { Peak timing } \\
\text { (days) }^{\mathrm{a}}\end{array}$} & \multicolumn{3}{|c|}{$\begin{array}{c}\text { Peak intensity } \\
\text { Category (cases/day/100,000) }\end{array}$} \\
\hline & $\begin{array}{l}\text { Prediction } \\
\text { date }\end{array}$ & $\begin{array}{l}\text { Time } \\
\text { to peak }\end{array}$ & $\begin{array}{l}\text { Prediction } \\
\text { error }\end{array}$ & Interpretation & Predicted & Actual & Interpretation \\
\hline $\begin{array}{l}2009 \text { (pandemic) } \\
\text { A (pH1N1) }\end{array}$ & $\begin{array}{l}27 \text { Sep } \\
2009\end{array}$ & 8 & -28 & Poor & $\begin{array}{l}\text { Medium } \\
(3.3)\end{array}$ & $\begin{array}{l}\text { Medium } \\
\text { (2.9) }\end{array}$ & Successful \\
\hline $\begin{array}{l}\text { 2010-11 } \\
B \text { and } A(p H 1 N 1))\end{array}$ & $\begin{array}{l}11 \text { Feb } \\
2011\end{array}$ & 10 & 0 & Excellent & $\begin{array}{l}\text { Medium } \\
(4.5)\end{array}$ & $\begin{array}{l}\text { Medium } \\
(4.9)\end{array}$ & Successful \\
\hline $\begin{array}{l}2011-12 \\
A(H 3 N 2)\end{array}$ & $\begin{array}{l}25 \text { Feb } \\
2012\end{array}$ & 9 & 7 & Good & $\begin{array}{l}\text { Medium } \\
((4.5))\end{array}$ & $\begin{array}{l}\text { Very high } \\
(12.4)\end{array}$ & Unsuccessful \\
\hline $\begin{array}{l}2012-13 \\
\text { A (H3N2), B and A } \\
\text { (pH1N1)) }\end{array}$ & $\begin{array}{l}22 \mathrm{Feb} \\
2013\end{array}$ & 10 & -7 & Good & $\begin{array}{l}\text { Very high } \\
\text { (10.1) }\end{array}$ & $\begin{array}{l}\text { Very high } \\
\text { (11.7) }\end{array}$ & Successful \\
\hline $\begin{array}{l}2013-14 \\
\text { A (H3N2), B and A } \\
\text { (pH1N1) }\end{array}$ & $\begin{array}{l}17 \text { Feb } \\
2014\end{array}$ & 8 & -8 & Acceptable & $\begin{array}{l}\text { Non-epidemic } \\
\text { (1.0) }\end{array}$ & $\begin{array}{l}\text { Medium } \\
\text { (3.4) }\end{array}$ & Unsuccessful \\
\hline
\end{tabular}

a. A positive value means that the peak was predicted to be reached before the actual peak occurs, whereas a negative value means that the peak is predicted after the actual peak occurs. 


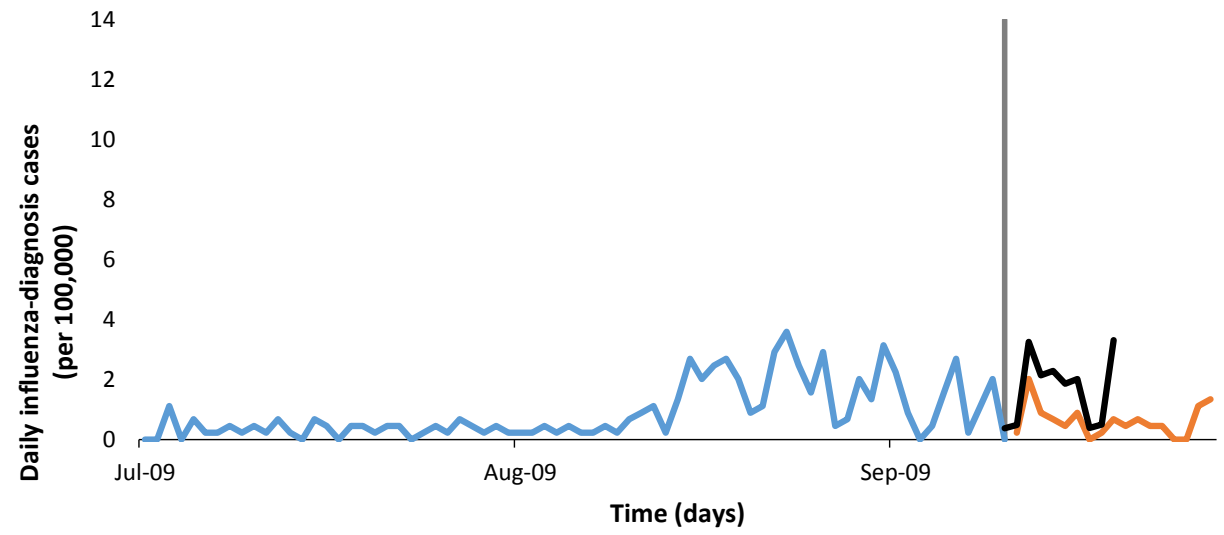

Figure 15. The prediction method applied to the 2009 pandemic (A (pH1N1)). The blue line represents the number of known actual influenza-diagnosis cases/day/100,000 when the prediction was made, the orange line represents the number of "unknown" actual influenzadiagnosis cases/day/100,000 when the prediction was made from the first "unknown" day and until the peak has passed, the gray bar marks the end of the known and the beginning of the "unknown" actual influenza-diagnosis cases/day/100,000, and the black line denotes the predicted values (using the peak intensity prediction) from the first "unknown" day until the predicted peak occurs.

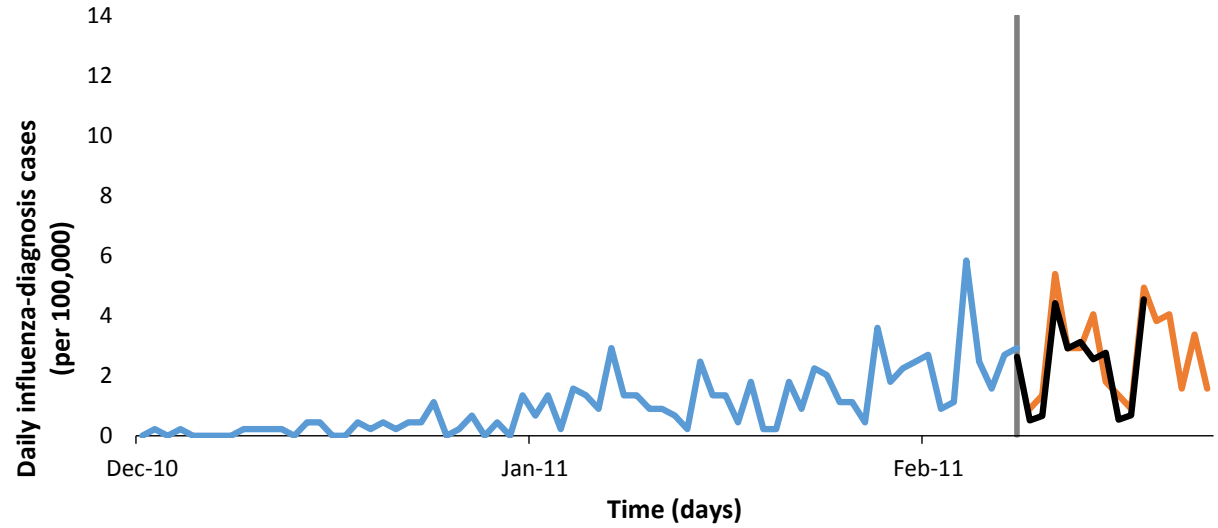

Figure 16. The prediction method applied to winter influenza season 2010-11 (B and A (pH1N1)). The blue line represents the number of known actual influenza-diagnosis cases/day/100,000 when the prediction was made, the orange line represents the number of "unknown" actual influenza-diagnosis cases/day/100,000 when the prediction was made from the first "unknown" day and until the peak has passed, the gray bar marks the end of the known and the beginning of the "unknown" actual influenza-diagnosis cases/day/100,000, and the black line denotes the predicted values (using the peak intensity prediction) from the first "unknown" day until the predicted peak occurs. 


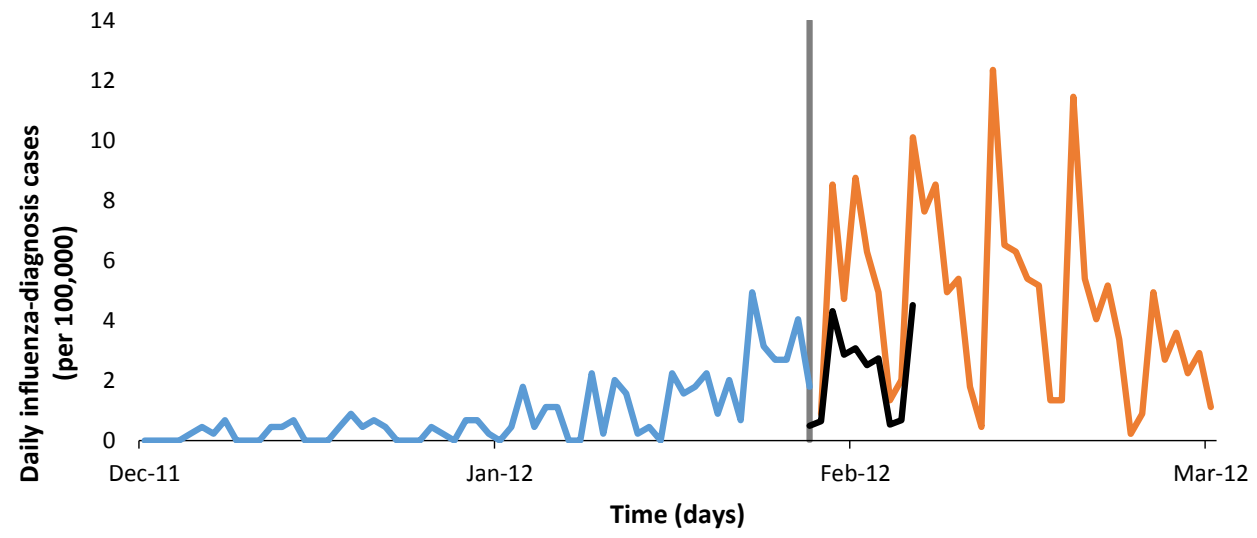

Figure 17. The prediction method applied to winter influenza season 2011-12 (A (H3N2)). The blue line represents the number of known actual influenza-diagnosis cases/day/100,000 when the prediction was made, the orange line represents the number of "unknown" actual influenza-diagnosis cases/day/100,000 when the prediction was made from the first "unknown" day until the peak has passed, the gray bar marks the end of the known and the beginning of the "unknown" actual influenza-diagnosis cases/day/100,000, and the black line denotes the predicted values (using the peak intensity prediction) from the first "unknown" day until the predicted peak occurs.

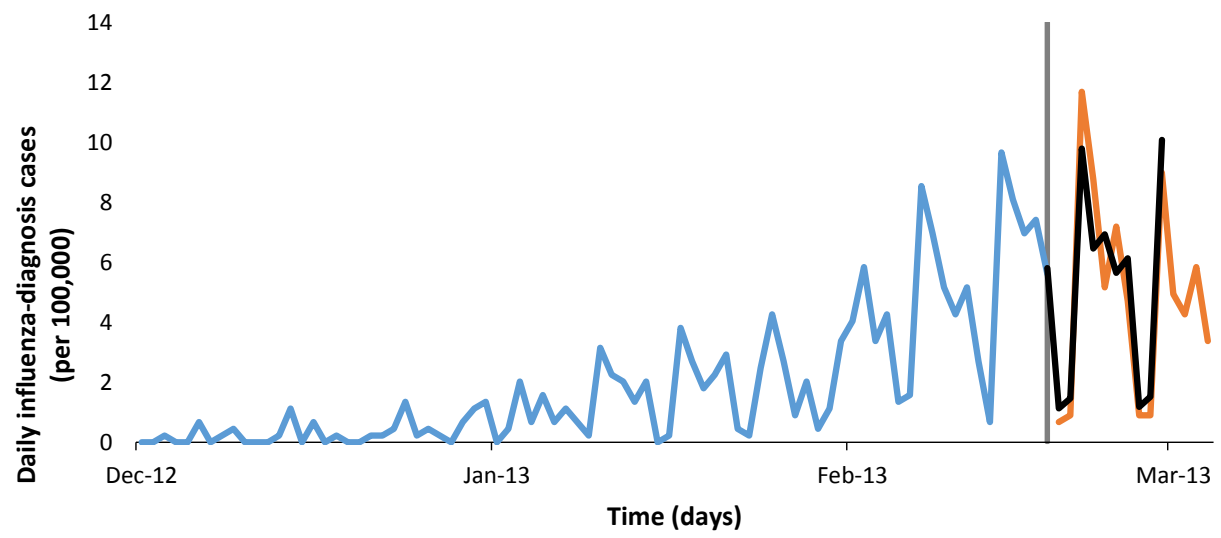

Figure 18. The prediction method applied to winter influenza season 2012-13 (A (H3N2), B and $\mathrm{A}(\mathrm{pH} 1 \mathrm{~N} 1))$. The blue line represents the number of known actual influenza-diagnosis cases/day/100,000 when the prediction was made, the orange line represents the number of "unknown" actual influenza-diagnosis cases/day/100,000 when the prediction was made from the first "unknown" day until the peak has passed, the gray bar marks the end of the known and the beginning of the "unknown" actual influenza-diagnosis cases/day/100,000, and the black line denotes the predicted values (using the peak intensity prediction) from the first "unknown" day until the predicted peak occurs. 


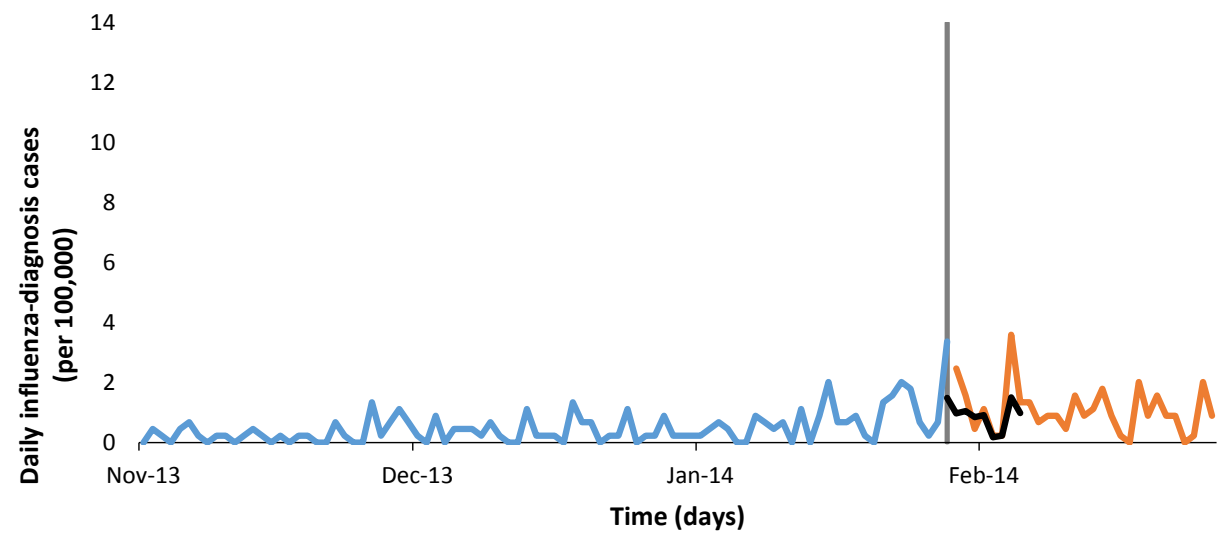

Figure 19. The prediction method applied to winter influenza season 2013-14 (A (H3N2), B and $\mathrm{A}(\mathrm{pH} 1 \mathrm{N1})$ ). The blue line represents the number of known actual influenza-diagnosis cases/day/100,000 when the prediction was made, the orange line represents the number of "unknown" actual influenza-diagnosis cases/day/100,000 when the prediction was made from the first "unknown" day until the peak has passed, the gray bar marks the end of the known and the beginning of the "unknown" actual influenza-diagnosis cases/day/100,000, and the black line denotes the predicted values (using the peak intensity prediction) from the first "unknown" day until the predicted peak occurs. 


\section{GENERAL DISCUSSION}

\section{Principal findings}

\section{Preparatory phase: examination of eHealth data sources}

In the eHealth study, the primary aim was to examine the associations (in terms of correlation) between eHealth data sources (GFT, telenursing data, and data from the local health service websites) and influenza-diagnosis data. The intention was to investigate the potential of using these sources as tools to improve the effectiveness of infectious disease surveillance in local settings. A secondary aim in this study was to investigate associations between eHealth data sources, media coverage, and the interaction between circulating influenza strains and age-related population immunity. Correlations with large effect sizes on a weekly basis were found between data from these eHealth sources and influenza-diagnosis data for both winter influenza seasons and the 2009 pandemic outbreak, with the exception of telenursing data during the winter influenza season in 2007-08 with influenza B and influenza A (H1) circulating. A study investigating the utilization of the Swedish telenursing service reported that young adults constituted a large group of callers (Wahlberg \& Wredling 1999). A possible reason for the underperformance of telenursing data during the winter influenza season in 2007-08 may be that this season comprised only a small proportion of young adults (Figure 2), which may have led to comparatively fewer individuals contacting the telenursing call service centers. For GFT, the outcomes are consistent with the findings of previous studies conducted at national, state, and local levels, which also reported strong associations between GFT and influenza-diagnosis cases (Ortiz et al. 2011, Malik et al. 2011, Dugas et al. 2012, Kang et al. 2013). Similar to telenursing data, in this study, GFT showed lower correlations with influenza-diagnosis cases for the 2007-08 and 2008-09 winter seasons when comparatively fewer young adults were diagnosed with influenza. Regarding website usage data from the local health service provider, the performance was similar to GFT for the two epidemics (2009 pandemic and 2011-12 winter influenza season) for which these data were collected. Even though the eHealth data sources and influenzadiagnosis cases showed strong associations throughout the study period, the time lag between these differed. While eHealth data tended to precede influenza-diagnosis data by 2 weeks during the first two winter influenza seasons (2007-08 and 2008-09), the time lag was reduced to 1 week or none from the 2009 pandemic onward. These findings indicate that eHealth data sources may be useful tools in influenza surveillance. However, before giving any 
recommendations, these sources must be used as syndromic data in influenza surveillance systems, followed by prospective evaluations of these systems.

\section{Preparatory phase: review and evaluation of existing detection and prediction algorithms}

In the meta-narrative review of studies evaluating the prospective performance of the influenza epidemic detection and prediction algorithms, only eight studies met the study criteria. Three of these studies evaluated seven different detection algorithms, and five of the studies evaluated nine different prediction algorithms. The methodological perspectives and experiences from algorithm evaluations have, over time, been reported in two distinct narrative formats: the biodefence informatics narrative and the health policy research narrative. The narratives were found to differ in elements ranging from the evaluation settings and endpoint measures used to structure the argument. With an emphasis on verification of technically and mathematically sound algorithms, the biodefence informatics narrative originates from the need to rapidly respond to evolving outbreaks of influenza pandemics and agents disseminated in bioterror attacks. Recent studies presented in the biodefence informatics narrative have been directed to common public health problems, such as winter influenza seasons and air pollution (Buehler et al. 2009). Only four prospective evaluations of influenza detection and prediction algorithms were reported as a health policy research narrative, despite the fact that evidence-based practices have been promoted by public health agencies during the period when the studies were published (ECDC 2011). On the other hand, although uncommon for influenza, algorithm evaluations emphasizing realworld validation of algorithm performance are quite common for several other infectious diseases, e.g., dengue fever (Hii et al. 2012). It is possible that the urgent quest for knowledge in association with atypical influenza epidemics has led to an acceptance of evaluation accounts with limited empirical grounding, which might explain why researchers choose not to report evaluations of influenza detection and prediction algorithms in the health policy narrative. In biodefence informatics, these accounts agree with mathematical and engineering research practices and are therefore accepted as scientific evidence within those domains. This indicates that, when interpreting algorithm evaluations, awareness of the narrative format in which evidence is reported is crucial.

The aim of the comparative accuracy trial was to evaluate and compare algorithms for the detection and prediction of increases in local influenza 
activity using two data streams (influenza-diagnosis data and syndromic telenursing data) from a county-wide health information system. In the study, three of the detection algorithms and three of the prediction algorithms identified in the meta-narrative review were prospectively evaluated using naturally occurring epidemics. Two modifications of one of the detection algorithms were also proposed and evaluated in this study. Among the detection algorithms evaluated in this comparative trial, only Serfling regression displayed a satisfactory performance when applied to influenzadiagnosis data during winter influenza seasons. Regarding the prediction algorithms, nonadaptive log-linear regression showed an acceptable performance both when applied to influenza-diagnosis data and to telenursing data. Among the remaining two prediction algorithms, an acceptable performance was only displayed for 2-weeks-ahead predictions for the naive method when applied to syndromic data. Previous studies have shown that parametric methods are not suitable when the parameters describing the incidence curve vary considerably from year to year (Andersson et al. 2008, Bock et al. 2008, Schiöler 2010), as is the case with winter influenza seasons (Timpka et al. 2012). However, the findings of the comparative trial suggest that Serfling regression, a parametric detection algorithm, and nonadaptive loglinear regression, a parametric prediction algorithm, may be considered, although carefully, when developing algorithms for use in influenza epidemic detection and prediction at local level. As a result, it was in this study concluded that both further evaluation research and research on a combination of these types of algorithms in public health information infrastructures for nowcasting (integrated detection and prediction) of influenza activity is warranted.

\section{Development phase: design and evaluation of the nowcasting method}

Following the findings in the previous phase, an influenza nowcasting method, using the same streams of data used in the previous studies was designed and presented. As a first step, the performance of this method was first evaluated using retrospective data, detecting the 2008-09 winter influenza season on the day it actually occurred, and predicting the peak timing and peak intensity in a satisfactory way. Although this method showed a promising performance in the retrospective performance evaluation, the conclusion was that thorough evaluations are warranted before the method could be recommended for wider use. Thus, a 5-year evaluation study was conducted in which the nowcasting method was prospectively evaluated using corresponding streams of data as in previous studies. In this prospective evaluation, both satisfactory aspects and 
areas where improvements are needed were identified. The detection module displayed a satisfactory performance throughout the evaluation period, except for the 2011-12 winter influenza season with influenza A (H3N2) circulating after a season with influenza B and influenza A (pH1N1) activity. Regarding peak timing predictions, the performance was satisfactory for the four winter influenza seasons evaluated, but not for the 2009 pandemic. The method categorized the local peak intensity levels correctly for the 2009 pandemic and for two of the winter influenza seasons, but it was unsuccessful in forecasting the very high peak intensity of the 2011-12 season and the medium peak intensity of the 2013-14 season that was preceded by a simmering phase.

\section{Definition of nowcasting}

In this thesis, the term nowcasting denotes integrated detection and prediction of influenza activity in the community. This interpretation is slightly different from the interpretation in the area from which the term originates, i.e. weather forecasting. In this area, nowcasting is used to denote integrated very shortterm predictions (a couple of hours) and long-term predictions. In addition, the interpretation also differs from the definition used in Donker et al. (2011) where nowcasting is used to denote situational awareness.

\section{Prospective evaluations of detection and prediction algorithms: the way to go}

In my opinion, one of the main issues in this field is that reports of detection and prediction methods lack proper evaluation procedures. Although a large number of studies have focused on developing these kind of methods, few studies have evaluated these using prospective data. In the meta-narrative review, several interesting studies were excluded because nonprospective data were used in the evaluations (Spreco \& Timpka 2016). Instead, in these cases, the evaluations were performed in retrospective settings. In some of the studies, methods were both developed and evaluated using the same dataset (e.g., Watkins et al. 2008, Kaufman et al. 2007), whereas other studies evaluated methods against out-of-sample verification data from the same dataset using a leave-one-season-out approach (e.g., Reich et al. 2015, Dugas et al. 2013). Although the latter may be considered valid from a mathematical perspective, it is far less appropriate from an epidemiological point of view. One of the reasons for this is because influenza epidemics do not act independently of each other; rather it is likely that recirculating strains from previous epidemics influence virus dissemination patterns during an epidemic. As a consequence, 
in one of the studies included in this thesis it was concluded that a retrospective evaluation is insufficient, regardless of the number of winter influenza seasons used in the evaluation. Instead, prospective evaluations are warranted, where historical data only are used to determine method parameters (such as alerting thresholds), and the method is applied to forthcoming epidemics.

\section{Evaluation metrics}

Although some consensus exists regarding the measurements to be used in the evaluation of detection algorithms (Unkel et al. 2012), this is unfortunately not the case for the evaluation of prediction algorithms. This has led to the situation whereby metrics of varied quality or no metrics at all are used to evaluate the performance of prediction algorithms. The same applies also for detection algorithms. Therefore, it is no surprise that numerous of studies were excluded from the meta-narrative review because they failed to evaluate detection and prediction algorithms using appropriate performance metrics (Figure 4) (Spreco \& Timpka 2016). For the evaluation of detection algorithms, timeliness, sensitivity, and specificity were used to assess the algorithm performance. In the comparative accuracy trial, these metrics were considered equally useful as performance metrics. In the development phase, all three metrics were used to interpret the algorithm performance in the retrospective setting, whereas only timeliness was used in the prospective setting. The reason for this was that the purpose in the retrospective setting was to find the optimal threshold, and this is easily determined by studying sensitivity and specificity in a ROC curve. On the other hand, the purpose in the prospective setting was performance evaluation of the method, and timeliness alone was considered sufficient as a metric. Based on extensive discussions during my $\mathrm{PhD}$ studies and on experiences from previous studies (Cowling et al. 2006, Biggerstaff et al. 2016), timeliness was ultimately considered to be the most valid general evaluation metric for the present purpose, whereas sensitivity and specificity were considered to be useful tools for deciding the optimal alerting threshold in the retrospective learning datasets. As a result, only timeliness was used to evaluate the performance of the detection module in the last study of this thesis.

Although sensitivity and specificity were not used as performance metrics, they still had a significant role in the process because they were used to decide the alerting threshold. One important concern to be solved regarding these two metrics is to determine the appropriate period of time on which these calculations should be based. In the comparative trial, the calculation of sensitivity was based on the first 10 weeks of an observed epidemic, whereas 
specificity was based on the 10 weeks immediately before an observed epidemic. However, in the nowcasting-design study, the calculation of sensitivity was based on the first 45 days of an epidemic and the specificity calculation was based on the period from when the previous epidemic ended until the beginning of the current epidemic (i.e., for specificity, the length of the period varied). This issue was discussed on several occasions during my $\mathrm{PhD}$ studies, hence the inconsistency between these studies. The rationale for limiting the time periods for both sensitivity and specificity in the comparative trial as well as the time period for sensitivity in the development phase was to avoid misleading higher performance values. This issue mainly concerns sensitivity, because once an epidemic has started, it is known that the incidence will exceed the predetermined threshold for a certain period ahead. Expanding this period would generate higher sensitivity and thereby overestimate the method performance. Similarly, if the periods are set too short, the performance of the method may be underestimated. Regarding specificity, there are two potential problems. A too long period may generate misleading high values because long inter-epidemic periods (when the incidence level is most often low or non-existing) are included in the calculations; a short period can lead to hypothetic increases in the incidence level during inter-epidemic periods being ignored in the calculations, leading to a higher value of the specificity, which also may be deceiving. The choice of the period length is therefore not absolute. However, if I am to give a recommendation, it would be that the calculation of specificity should be based on the period from when the previous epidemic has come to an end until the beginning of the current epidemic, whereas the sensitivity calculation should be based on a relatively short time period, but the exact length I leave unsaid. Also note here that weekly data were used in the comparative trial, whereas daily data were used in the development phase. More on that in the Data section.

As mentioned, there seems to be no consensus regarding evaluation performance metrics for prediction algorithms. Therefore, it is not surprising that a large variety of metrics are used when evaluating prediction algorithms. For example, some researcher have used a combination of positive predictive value (PPV) and area under the curve (AUC) (Timpka et al. 2014a); others have used a combination of median absolute deviation (MAD), median absolute percentage error (MedAPE) and root-mean-squared error (Burkom et al. 2007) or Pearson's correlation coefficient ( $r$ ) (Viboud et al. 2003). The choice of metric also depends on what is predicted. In this thesis, the progress of an epidemic was predicted in the comparative trial, whereas in the development phase, the 
peak timing and peak intensity were predicted and therefore the metrics varied in these studies. In the comparative trial, the combination of Pearson's correlation coefficient and MedAPE was chosen, where Pearson's correlation estimated how well the predicted time series followed the observed time series and MedAPE estimated the deviance between the observed and the predicted levels of the time series. In theory, these two metrics apparently complement each other very well and are therefore believed to provide a sufficiently valid and accurate description of the predictive performance. In the development phase, timeliness was used to evaluate the peak timing prediction, whereas slightly different metrics were used to evaluate the peak intensity predictions in the retrospective and in the prospective settings. For logical reasons, in the first, an exact metric (the relative difference between the predicted peak intensity and the actual peak intensity) was used, whereas in the latter, the predicted peak intensity was compared with the correct peak intensity category defined by Vega et al. (2015).

\section{Interpretation limits of the evaluation metrics}

In the three studies in the preparatory phase, Pearson's correlation coefficient was used to interpret the association between different data sources (applies for the eHealth study) or the predictive performance of influenza prediction algorithms where the predicted time series is compared with the actual one (applies for the meta-narrative review and the comparative trial). In the eHealth study, the limit values were applied as suggested by the Cohen scale (Cohen 1988). This scale defines small, medium, and large effect sizes as $0.10,0.30$, and 0.50, respectively. However, these limits may not be semantically optimal for use in these studies. The limits are applicable in settings where data contained in multiple explanatory variables are used to explain the variance in one response variable, but not so well suited in settings where only two parallel data streams are compared. These limits were therefore modified in the metanarrative review and the comparative accuracy trial, and set at $0.90,0.80$, and 0.70 for analyses of weekly data, and $0.85,0.75$, and 0.65 for daily data, denoting outstanding, excellent, and acceptable predictive performance, respectively. These limits are far more appropriate when using the metrics in this context. For the remaining metrics used in the meta-narrative review and the comparative trial (see Table 1), to my knowledge there are no comparable standard limits. These limits were therefore carefully set by the authors of these papers. Having nothing to compare with, the limits are considered suitable, until proven otherwise. 
The limits used to interpret the timeliness in the two studies in the development phase stand well in contrast to the limits used in the CDC challenge study (Biggerstaff et al. 2016). In this study, forecasts of the start and peak of an epidemic were based on weekly data and considered accurate if they occurred within 1 week of the actual timing of each component, whereas forecasts in the two studies in the development phase were considered excellent if the timeliness was up to 3 days and good if it was $4-7$ days. These limits are therefore considered to be at least as strict and accurate as those used in the CDC challenge. With regard to the prediction of the peak intensity, a forecast was considered to be accurate if it predicted the peak intensity to the correct peak intensity category defined by Vega et al. (2015). In that study, the thresholds for each of these categories were calculated for every winter influenza season by applying the moving epidemic method (Vega et al. 2013) to five to ten previous influenza seasons. Hence, these categories are considered to be reliable.

\section{Data}

\section{Data sources}

It has been stated that methods based on syndromic surveillance data may serve as complements to traditional surveillance by provision of earlier indications of influenza activity (Friesema et al. 2009, Ma et al. 2015). Following this, the potential of using several different syndromic data sources as a tool in local influenza surveillance was examined at the beginning of this thesis (in the eHealth study). The findings were promising and corresponded to the recent outcomes worldwide (Kim et al. 2013, Timpka et al. 2014a, Nagel et al. 2013, Yom-Tov et al. 2014, Kirian \& Weintraub 2010, Socan et al. 2012). However, it was concluded that further longitudinal research incorporating prospective evaluations of actionable alerts (Milinovich et al. 2014) is required before eHealth surveillance systems can be used in routine public health practice. For that reason, in this thesis, both existing methods and the nowcasting method were applied to prospective syndromic data and naturally also to traditional influenza data. Data on clinical influenza-diagnosis cases were used as gold standard data, and data on telenursing chief complaints were mainly used as syndromic data. The first was chosen as gold standard data instead of laboratory-diagnosis data because, compared with other regions in Sweden, relatively few, suspected cases of influenza are analyzed in laboratories in Östergötland County. However, even if a larger number of samples was analyzed, clinical influenza-diagnoses would still be preferred because 
precious time is lost before laboratory data are available for algorithmic processing.

Regarding syndromic data, there are two main reasons for choosing to use data on telenursing chief complaints. First, telenursing data are available on a daily level, which is not the case for, e.g., GFT data. Second, the 2-week (when using weekly counts) and 14-day (when using daily counts) preceding time lag between telenursing chief complaints and influenza-diagnosis cases were most strongly associated among the available eHealth data sources previously investigated (Timpka et al. 2014a, 2014b). Because the main aim of this thesis was to predict the start and the peak timing of an epidemic as early as possible, these two motives were crucial. The findings in the eHealth study indicate that, when using weekly counts, telenursing data were better associated with influenza-diagnosis data for two of the winter influenza seasons when telenursing data preceded influenza-diagnosis data by 1 week and not 2 weeks. However, the numerical differences in terms of correlation between these two time lags were negligible. Among the time lags investigated between influenzadiagnosis data and telenursing data using data on daily counts (1-14 days), the strongest association occurred when telenursing data preceded influenzadiagnosis data by 14 days (Timpka et al. 2014a). Another reason for choosing telenursing chief complaints as syndromic data is that complaints such as fever and cough are less likely to be affected by media publicity than data reflecting Internet activity. However, there is a risk that telenursing data may not be fully appropriate if the circulating virus strains mainly affect older age groups, because the use of telenursing services in these groups is lower (Timpka et al. 2014b). An alternative would be to use data on over-the-counter drug sales (Liu et al. 2013), but the availability of these data in real time is limited in many countries, including Sweden. Regardless, these observations suggest that regular upkeep of the syndromic data sources and validation against clinical and laboratory data are necessary when using eHealth data in influenza surveillance.

\section{Temporal granularity of data}

In this thesis, both weekly and daily counts of data were used. In the comparative accuracy trial, weekly data were used to evaluate and compare the performance of existing detection and prediction algorithms, whereas in the evaluation of the nowcasting method, daily data were used. The temporal data chosen in each study depended on the purpose of the study. In the comparative trial, the aim was to compare methods and, for this purpose, it was considered 
important to have the same temporal data and not necessarily as important to have specific temporal data, whereas in the nowcasting method evaluation, as detailed temporal data as possible was required because the aim was to detect the beginning of an epidemic and the peak timing and intensity as rapidly as possible.

\section{The nowcasting method design: comparison with previous work}

Many essential policy decisions in the response to increased influenza activity are made at the local level, e.g., planning of resources in intensive care units and deciding social distancing measures such as school closures. The design of the nowcasting method presented in this thesis can be compared with current state-of-the-art Big Data approaches to influenza forecasting (Biggerstaff et al. 2016). The multi-linear autoregressive framework is one such approach for local application. In this framework, the information is combined from several different data sources, ranging from GFT to electronic patient records (Goel et al. 2010, Shaman et al. 2013, Shaman \& Kandula 2015, Yang et al. 2014, Yang et al. 2015). In this thesis, full-season learning periods for model updates are used, whereas in the multi-linear autoregressive framework continuous updating of the model during an epidemic is used. Seasonal updates were chosen because a central design criterion was to highlight the transparency of the method construction and hence simplify implementation by other researchers. Adjustments to the algorithm processes were only made before pandemics and during winter influenza seasons to adjust detection thresholds and, if necessary, the levels for simmering low influenza activity. Methods for continuous updating most often rely on the local data characteristics, which makes it challenging to define a continuous updating framework in a decontextualized format sufficiently transparently to enable transmission of the framework to other local settings and at the same time uphold similar performance levels.

In the chosen approach in this thesis, a detection component is added, which is another difference from the multi-linear autoregressive framework. In weather forecasting, combined nowcasting methods (including both detection and prediction components) are common. However, this is unfortunately not the case in infectious disease epidemiology, which is rather surprising. Although numerous studies have focused on developing either influenza detection or influenza prediction algorithms, few have focused on combining these (Spreco \& Timpka 2016). One possible explanation for this may be that researchers have 
attempted to develop integrated nowcasting methods, but failed to obtain satisfactory outcomes and therefore have chosen not to publish the method designs. However, even if the results are unsatisfactory, the methods should be published so that other researchers can further develop and improve them.

\section{Strengths and limitations}

\section{Preparatory phase}

Examination of eHealth data sources

The strength of the eHealth study is that it compares three eHealth data streams over a 5-year period, including winter influenza seasons and a pandemic influenza outbreak, and identifies cross-correlations and time lags for the different epidemics. Because the population of Östergötland is fairly representative of Sweden as a whole, it is possible to generalize the findings, although with care, to communities in settings with a similar North-European population and geographical characteristics. However, there are also important limitations that should be taken into consideration when interpreting the results. One limitation is that cases of influenza were defined by clinical diagnosis and not laboratory-confirmed cases. The latter data source was restricted to a limited period and only used for validation purposes. On the other hand, the effect size of the correlation between the microbiological and clinical diagnosis rates observed was large during the validation period, and corresponded to the findings reported from other settings (Ortiz et al. 2011). Another limitation is that different strains of the influenza virus affect different age groups. This may make eHealth surveillance less reliable during winter influenza seasons when the circulating influenza strains mainly affect the elderly in the population. Additional limitations with the eHealth data sources are discussed in the "Syndromic data" section.

\section{Meta-narrative review and comparative accuracy trial}

One strength of the meta-narrative review is that this approach is a relatively new method of systematic analyses of published literature, designed for topics that have been conceptualized differently and studied by different groups of researchers (Greenhalgh et al. 2005). From a historical perspective, it was found that researchers from diverse paradigms have evaluated influenza detection and prediction algorithms by different means and with different intentions. Some researchers have conceptualized algorithm evaluations as a subarea of epidemiology, others as an engineering discipline. The intent of this review was to summarize different perspectives on algorithm development and reporting in overarching narratives and not to conclude recommendations for algorithm 
use. The purpose of this was to highlight what different researchers might learn from one another's approaches.

With regard to the limitations of the meta-narrative review, there is a possibility that some relevant evaluations were not identified. This could have been the case, for instance, when specific indexing terms for infection disease detection and prediction methods were absent, so that studies that were not indexed in the research databases were missed. However, because the initial search yielded 1084 studies, the probability that relevant evaluations were not identified is low. It must also be taken into consideration that the aim was to base the narrative analysis on evaluations with relevance for operational readiness and real-world application. Therefore, studies lacking these characteristics were disregarded. Of the 1084 studies identified, 116 had relevant abstracts. Nevertheless, most of these were excluded from the final review because they did not fulfil the inclusion criteria at the detailed level (Figure 4), giving the overall impression that more research activity had been associated with developing detection and prediction algorithms than evaluating them and carefully reporting the results. For instance, some interesting studies were excluded because nonprospective data were used for the evaluations (e.g., Reich et al. 2015, Dugas et al. 2013), and other studies had to be excluded because standard evaluation metrics were not reported (e.g., Ohkusa et al. 2011). The fact that no study applying an integrated influenza detection and prediction method was found may be seen as a limitation. However, I consider this to be a limitation of the field and not a limitation of the meta-narrative review. Although Boyle et al. (2011) applied an algorithm including both detection and prediction components, this study was excluded because simulated data were used for the evaluation. In addition, syndromic definitions of influenza as the gold standard, i.e., specified sets of symptoms not requiring laboratory confirmation for diagnosis (Last 2001), were accepted in this review. If only laboratory-confirmed influenza-diagnosis was accepted as gold standard data, almost no studies would have qualified for inclusion in the review.

Regarding the comparative accuracy trial, it is one of the first studies to evaluate influenza detection and prediction algorithms in a prospective setting using real empirical data. Because the characteristics of baseline conditions in public health practice, such as temporal patterns and noise, are likely to have an influence on algorithm performance, the use of real data for algorithm evaluations is recommended (Buckeridge et al. 2008). Concerning the 
limitations, only data from one season were used for algorithm learning because long-term data series of constant quality are seldom available presently at local public health levels. For this reason, several interesting algorithms were excluded, and some of the evaluated algorithms would probably have given a more satisfactory performance if the learning data had covered a longer period of time. This shortage of longitudinal data is a known problem in the evaluation of influenza detection systems (Lotze et al. 2011). To solve this data scarcity problem, data simulation has been used. However, the main remaining challenge is replicating the complexity of both epidemic data streams and baseline data (Buckeridge et al. 2005a, Buckeridge 2007). Correspondingly, algorithms reported only from theoretical settings were excluded, with similar implications.

Additional strengths and limitations in these two studies have been discussed earlier, i.e., the lack of prospective evaluations in influenza surveillance, the strengths and weaknesses of the evaluation metrics used, and their interpretation limits, etc.

\section{Development phase: design and evaluation of the nowcasting method}

The nowcasting method developed in this thesis has apparent strengths but also limitations that need to be taken into account. One of the main strengths is that the design rationale is documented in detail to allow other researchers to consider the arguments for diverse design decisions when building the next generation of nowcasting methods. Another important strength is that an epidemic is considered to consist of three different components (beginning of the epidemic, peak timing of the epidemic, and peak intensity of the epidemic), where statistical and mathematical assumptions for each of these components are made independently of each other. In addition, different data sources are used in each component. Specifically, exponential regression is applied to influenza-diagnosis data to detect the beginning of an epidemic, simple linear regression is applied to syndromic telenursing data to predict the peak timing, and a derivate of the normal distribution density function is applied to influenza-diagnosis data to predict the peak intensity following the assumption that the epidemic follows a bell-shaped function of time around the peak. To my knowledge, a similar approach has seldom been reported in the influenza surveillance field. An additional key strength is that the developed method is one of the first to be prospectively evaluated using longitudinal local data from naturally occurring influenza activity. 
The nowcasting method also has limitations that need to be considered when interpreting the results. First, the series of actual influenza-diagnosis data are smoothed and the peak of the smoothed series is considered as the actual peak when comparing it with the predicted peak. However, the reason for this design choice was to reduce the risk of misleading impact from outliers. Second, in the peak timing prediction component, linear regression was applied to 7day periods in the search for positive and negative trends in order to find the peak timing in the syndromic data. The length of the period could have been extended by 1-2 days to get more robust estimates of the trend. On the other hand, this was weighted against the risk of predicting the influenza-diagnosis peak timing fewer days in advance, and the benefit with earlier prediction of saving these days was preferred. Third, the peak intensity prediction is affected by the peak timing prediction. The probability of an accurate prediction of the peak intensity increases if the peak timing prediction is precise. For example, if the timeliness of the peak timing prediction was 0 days instead of 7 days in the retrospective evaluation of the 2008-09 winter influenza season, the predicted peak intensity would have been estimated at 7.7 instead of 6.3 influenzadiagnosis cases/100,000 compared with the actual 8.5 influenza-diagnosis cases $/ 100,000$, i.e., the absolute difference between the predicted and the actual incidence would have been $10 \%$ instead of $26 \%$. Fourth, in the peak intensity prediction component, an influenza epidemic was assumed to take a bellshaped form from the beginning to the end and therefore a derivate of a normal distribution density function was used to estimate the peak intensity. Bregman \& Langmuir (1990) used the same assumption to predict the course of the AIDS epidemic in the United States, but this was later shown to be inaccurate (Nishiura 2007). However, the intention in Bregman \& Langmuir (1990) was to predict the course of the AIDS epidemic, which had been ongoing for several years in an ill-defined population, whereas in this thesis, the bell-shaped function was only used to find the peak intensity in an increase of influenza activity that lasted for only one season. In other words, the underlying premises radically differ from each other in these two settings. In the context and for the purposes used in this thesis, the assumption of a bell-shaped curve is considered defensible.

Moreover, an additional limitation concerns epidemics which deviate from a regular-shaped curve, because the nowcasting method is based on the assumption that local influenza activity with relevance for health care planning normally follows a regular epidemic shape. Although measures were taken to 
deal with deviations from this postulation, the method displayed, in varied ways, poorer predictions during the two epidemics that were foreseen to deviate from a regular shape, i.e., the 2009 pandemic and the simmering 201314 winter influenza season. The choice to double the detection threshold before applying the detection algorithm in the pandemic and simmering settings proved to be successful. This adjustment of the threshold led to a satisfactory performance for the two affected epidemics; if the threshold had not been doubled, the performance of the algorithm would have been poor (timeliness 24 days for the pandemic and 40 days for the 2013-14 winter influenza season). However, the peak timing prediction was poor for the pandemic and the prediction of the peak intensity level was unsuccessful for the simmering 201314 season, which is an indication that the current assumptions in the statistical modeling and settings were inadequate to deal with performance associated with deviating epidemics. For instance, the 2009 pandemic consisted of two waves, whereas in regular winter influenza seasons only one wave normally occurs. The nowcasting method is, however, designed to only estimate the first peak, while the second peak is disregarded. This could possibly be handled by investigating indications of important societal changes, e.g., timing of school closures or vacations and fluxes of travels (Merler et al. 2011), together with the use of the nowcasting method. If social distancing methods are applied in association with the arrival of a pandemic virus, there can be reasons to assume that epidemics may display two or more waves. Another way to handle such situations could be by following the pattern of the preceding syndromic data series with the intention of identifying a second increase in an epidemic once the first wave has decreased. For example, a second wave is evident in the telenursing data for the 2009 pandemic (Figure 9). In general, these findings imply that it is essential to study pre-epidemic activities to identify deviating patterns and make appropriate adjustments before applying a model-based method to an epidemic.

One weakness concerns the performance of the nowcasting method during the 2011-12 winter influenza season. During this season, both the detection function and the peak intensity prediction function underperformed. One possible reason for this is that the data from preceding epidemics used to generate the epidemic curves were insufficient for modeling the between-seasons drift in the immunity status of the local population in relation to the influenza strain(s) in circulation. In other words, it is possible that the current parameters used to compute the epidemic curves are inadequate when big changes in virus dissemination patterns occur. For example, it is likely that the epidemic phase 
of the influenza A (H3N2) winter influenza season in 2011-12 did not start with virus spread among the younger population in the community as during previous influenza seasons (Schanzer et al. 2011, Timpka et al. 2012). Inclusion of virologic information (i.e., influenza type and subtype) as model parameters may improve the predictive precision of mathematical models, as stated in a recent study (Moniz et al. 2016). Also inclusion of the population age structure as a model parameter may be considered. Nevertheless, such model extensions are only realistic if data on the added parameters are available in the local settings where the models are to be applied.

Furthermore, it was assumed that the syndromic telenursing data preceded the influenza-diagnosis data by 14 days. Although this is a grounded assumption (Timpka et al. 2014a, 2014b), the time lag may change over time. Using a constant 14-day time lag, the influenza-diagnosis peaks for the 2011-12 and 2012-13 winter influenza seasons were estimated 1 week before and 1 week after the actual influenza-diagnosis peaks. These findings raises suspicion that the assumption that telenursing data precedes influenza-diagnosis data by 14 days is not always appropriate. Therefore, it is possible that the peak intensity predictions would have been more accurate if the peak timing predictions were more precise. Recently, it was reported that methodologies supporting the use of digital data sources (e.g., search query logs) need regular upkeep to maintain their accuracy (Althouse et al. 2015), and the same surely applies to the use of syndromic data sources (such as telenursing data). In upcoming versions of nowcasting methods, both regular updates and more stable sources than telenursing data (with regard to the time lag to influenza-diagnosis data) may become accessible and could be used to improve the peak timing predictions and thus also the peak intensity predictions. Finally, a longer prospective evaluation period would have increased the possibility of drawing valid conclusions regarding the outcome of the evaluation, and it would be preferable to have corresponding local data from other cities or regions (Moss et al. 2017). Evaluations of the nowcasting method on epidemics from several other regions would allow conclusions to be drawn about the generalizability of the method.

Other strengths and weaknesses of the development phase have been discussed earlier, including the evaluation metrics used and their interpretation limits. 


\section{Syndromic data}

The main strength regarding syndromic data sources is that it has been shown previously that syndromic data precede gold standard data (influenzadiagnosis cases) by up to 14 days (Timpka et al. 2014a, 2014b). These findings suggest that syndromic data have the potential to be used in influenza surveillance systems. However, there are also several limitations that need to be taken into consideration. Although data from telenursing call centers and website usage data from health service providers were collected prospectively, the GFT data were downloaded in 2012. There is a possibility that the GFT data for 2007-11 had been retrospectively adjusted to better correlate to recorded clinical influenza-diagnosis cases. Although it has been reported that GFT algorithms are recalibrated every year (Wilson et al. 2009, Butler 2013), it is not evident whether or not these recalibrations influence the transformed Web query data available for download. Another issue with GFT data is that they are only available on a weekly level, which makes it impossible to use them for monitoring on a daily basis. Furthermore, the telenursing data were based on chief complaint codes defined for Sweden. Some complaints, such as fever and cough, were coded as age-specific syndromes, and other complaints were age neutral. Telenursing chief complaint coding also differs worldwide. For example, in a Canadian study evaluating the telehealth system used there, the chief complaint of cough is divided into two categories; acute productive and acute nonproductive (Rolland-Harris et al. 2012) while this is not the case in Sweden. To allow comparisons between telenursing systems and to facilitate valid and reliable recording, internationally standardized telenursing complaint codes are desirable.

In the comparative accuracy trial, the performance of the detection algorithms was poor when applied to syndromic telenursing data. One reason for this is that most of the detection algorithms evaluated were threshold based, whereas the baseline of the telenursing data had an increasing trend. For instance, the average number of telenursing calls during the inter-epidemic period before the winter influenza season in 2011-12 had increased by nearly $18 \%$ compared with the corresponding number of calls during the intermittent period before the winter influenza season in 2010-11 (Figure 1). When calibrating the algorithms using the learning set of telenursing data, the thresholds were set lower than would have been optimal for the algorithms to perform well during the evaluation period. This was particularly reflected in the sensitivity outcomes because low thresholds that lack empirical foundation make the calibrated algorithms excessively sensitive for raising alarms. It has been 
emphasized previously that regular preprocessing of syndromic data, correcting for issues such as daily autocorrelations, seasonal trends and day-ofthe-week effects, is required (Lotze et al. 2008). For example, in Timpka et al. (2014a), a baseline temporal trend of telenursing calls was estimated in the retrospective data using linear regression $\left(y=b_{0}+b_{1} t\right.$, where $y$ is the incidence, $b_{0}$ is the intercept, $b_{1}$ is the slope, and $t$ is the time unit) and corrected for. In addition, it was shown that algorithms perform best in specific settings when applied to syndromic data, e.g., depending on the shape of the epidemic signal (Buckeridge et al. 2005b). This implies that prospective application of detection algorithms on syndromic data is complex and requires consideration of preprocessing the data streams and combining detection approaches, and not only applying one best algorithm on unprocessed unidimensional data (Dórea et al. 2014). However, combined approaches may lead to decreased specificity for the system as a whole (Dohoo et al. 2010). 


\section{IMPLICATIONS FOR PRACTICE}

As mentioned, the term nowcasting was initially introduced in the field of meteorology to denote short-time forecasting. World Meteorological Organization (WMO 2017) describes nowcasting as follows:

"Nowcasting comprises the detailed description of the current weather along with forecasts obtained by extrapolation for a period of 0 to 6 hours ahead. In this time range it is possible to forecast small features such as individual storms with reasonable accuracy. A forecaster using the latest radar, satellite and observational data is able to make analysis of the small-scale features present in a small area such as a city and make an accurate forecast for the following few hours./..../ The strength of nowcasting lies in the fact that it provides location-specific forecasts of storm initiation, growth, movement and dissipation, which allows for specific preparation for a certain weather event by people in a specific location." 1

The key term here is location-specific forecasts. In influenza surveillance, nowcasting is a novel methodological approach that can be integrated in modern health information systems to detect and predict local influenza activity. Health care providers can start utilizing customized nowcasting methods locally and not only rely on forecasts made using national influenza surveillance systems and data. Based on local assumptions and using local influenza data, health care providers can design influenza nowcasting methods that are tailored for the specific local settings where the methods are to be applied and then apply the methods in these settings. The availability of accurate forecasts in local settings would enable health care providers to take more appropriate preventive actions, such as providing bed spaces and preparing ICU resources for severe influenza cases, than would have been possible only using national forecasts.

\footnotetext{
${ }^{1}$ World Meteorological Organization (WMO). Nowcasting. WMO 2017.
} 


\section{FUTURE WORK}

\section{Formulation of standardized reporting criteria}

A large number of studies have focused on developing and attempting to evaluate influenza detection and prediction algorithms. Although many of these studies offer interesting methodological approaches, the meta-narrative review revealed that they often fail to either evaluate the algorithms developed or to report their performance (or both) properly (Spreco \& Timpka 2016). For example, this review identified several studies that evaluate methods in retrospective settings or fail to report standardized performance metrics. Based on this, experts from epidemiology, mathematics, and statistics, as well as experts from other relevant fields, should focus on defining and formulating standardized and transparent reporting criteria for all types of algorithm evaluation research. Recently reported consensus statements for evaluations of prognostic models in clinical epidemiology (Collins et al. 2015) and the review on statistical methods for prospective detection of infectious disease epidemics (Unkel et al. 2012) can be used as references when formulating reporting criteria in the influenza surveillance field. Standardized scientific criteria of this kind enable researchers to learn from other researchers' approaches and also simplify replication of one another's designs and methods. By doing this, scientists can more easily draw generalized conclusions about existing approaches and methods, which can eventually lead to categorization of methods that are applicable in general and methods that are only applicable in local settings.

\section{Further development and evaluation of the nowcasting method}

Previously in this thesis, it was contended that although the performance of the nowcasting method was promising, there are still areas where improvements are warranted. For example, additional parameters could be included in the models used in the nowcasting method. It is possible that the unsatisfactory performance during the 2011-12 winter influenza season was because the epidemic phase of this season did not start with virus spread among the younger population in the community as occurred in previous winter influenza seasons (Schanzer et al. 2011, Timpka et al. 2012), and therefore virologic information could be included as parameters in the model. Another possible improvement could be to include population age structure as a variable in the 
model. The nowcasting method could be applied to specific groups, for example, the age group with the highest risk of being infected, instead of the total population. Evaluations on models including variables on age structure and virology as well as possibly information on gender, ethnicity (e.g., nativeborn or foreign-born), residency (urban or rural), and other relevant variables should be performed in the future. However, for this to be possible, data on these variables must be made available in the settings where the models are to be applied.

When appropriate variables are found, they should be included in the nowcasting method. The method should then be applied and further evaluated in multiple local settings, including large urban areas, to investigate the validity of the method. However, the present method (i.e., without extensions) should also be evaluated in other local settings before adding variables because it is possible that the method with the current set of variables may work better in areas other than the local settings evaluated in this thesis. If the nowcasting method generates promising results after thorough prospective evaluations, it should be recommended for use in public health surveillance systems.

\section{Application of the nowcasting method on other syndromic data}

In this thesis, the nowcasting method was applied to syndromic telenursing data, in addition to diagnostic data. However, as previously discussed, the assumption that telenursing data precedes influenza-diagnosis data by 14 days is not always appropriate and may contribute to less valid predictions of the peak timing and the peak intensity. More stable syndromic data sources (with regard to the time lag to influenza-diagnosis data) are therefore warranted. Findings in the eHealth study indicate that other data sources may also be used as syndromic data for influenza surveillance, such as data from health service websites (Timpka et al. 2014b). Other interesting data sources include over-thecounter drug sales, logs from Twitter and logs from Google Analytics. Although the eHealth study showed that GFT data are strongly associated with diagnostic data, use of this source as a substitute for telenursing data is not recommended because GFT data are only available on a weekly basis and they may also be retrospectively adjusted to better correlate to diagnostic data. Consequently, I recommend that the nowcasting method is evaluated using Google Analytics logs or data from health service websites as syndromic data, together with influenza-diagnosis data as the gold standard data. 


\section{Implementation of the nowcasting method}

Finally, if the present nowcasting method, or a modified version of it, proves to be valid in multiple local settings it should be implemented and used for influenza monitoring by the county councils. However, for this to be possible, daily counts of both the gold standard data and syndromic data used must be available on a daily basis and accessible as soon as possible, preferably the next day. 


\section{CONCLUSIONS}

In the study examining the performance of eHealth data sources in local influenza surveillance correlations with large effect sizes between influenzadiagnosis data and eHealth data were found. With these findings in mind, the main practical inference of this study is that further longitudinal research incorporating prospective evaluations of actionable alerts (Milinovich et al. 2014) is required before eHealth data sources can be used as influenza monitoring tools in routine public health practice. Following this conclusion, a meta-narrative review was conducted in which two narratives for reporting prospective evaluation of influenza detection and prediction algorithms were identified. The health policy research narrative is used to allow conclusions to be drawn about public health policy, whereas in the biodefence informatics narrative, mathematical and technical verification of algorithms are described. Two suggestions for future research and development were offered in the metanarrative review. First, a system of evaluation research phases connected by a translation process should be defined, starting from theoretical research on the development of new algorithms in the biodefence informatics setting and proceeding stepwise to prospective evaluations performed as health policy research. Second, standardized and transparent reporting criteria should be formulated for all types of algorithm evaluation research.

In the comparative accuracy trial, it was found that some of the algorithms identified in the meta-narrative review display a satisfactory performance when applied on influenza-diagnosis data during winter influenza seasons in local public health settings. Regarding syndromic data, the performance of the algorithm was satisfactory for prediction but unsatisfactory for detection. During the 2009 pandemic outbreak, the performance of the algorithm was generally poor. Based on the promising performance of Serfling regression as a detection algorithm and nonadaptive log-linear regression as a prediction algorithm, it was concluded that further evaluation research and research on combinations of these types of methods for nowcasting (integrated detection and prediction) of influenza activity are warranted.

In an attempt to respond to the first suggestion of the meta-narrative review and following the findings in the comparative accuracy trial, a nowcasting method was designed and prospectively evaluated on naturally occurring local epidemics in the development phase of this thesis. The nowcasting method developed is one of the first designed for application on naturally occurring 
local influenza epidemics. In the evaluation using prospective data, the method showed a promising performance, leading to the natural inference that it has potential for routine use. However, improvements are needed in some areas. For example, the models used in this type of method should be extended to include information on present and previous influenza types in circulation as well as information on the age distribution of the population. Before the nowcasting method (with or without extensions) can be recommended for use in public health surveillance systems, its validity must be investigated by application and further evaluation in multiple local settings, including large urbanizations. 


\section{ACKNOWLEDGEMENTS}

In the name of God, most gracious, most merciful. All praise be to God, the Lord of all living things. I am everlastingly thankful for everything You have given me and protected me from!

Many people have influenced and helped me to get to this point, too many to mention individually. However, there are some I wish to thank in particular.

My supervisor Toomas Timpka - You have been and still are a great source of inspiration. It has been a privilege to have you as a supervisor and to be part of your research team. You have introduced me to and guided me through the frightening world of science and academia. We have discussed diverse topics throughout the years, including important ones such as soccer. It was particularly a pleasure to comment on soccer games with $x$ weeks of time lag. And although you tend to choose wrong teams to support, I still consider you a very dear friend. Thank you for everything!

My co-supervisor Olle Eriksson - You have patiently provided support and brilliant insight into statistics. Your impressive skills in statistics have most often left me amazed. You have always offered solutions to the problems I caused! Many thanks for contributing to increase my knowledge!

My co-supervisor Örjan Dahlström - Your remarkable expertise in multiple disciplines, such as mathematics, statistics, behavioral science and public health, are to say the least inspiring. Thank you for providing invaluable advice. I have enjoyed our discussions.

Eva Blomqvist, Joakim Ekberg, Henrik Eriksson, Einar Holm and Magnus Strömberg - I have gained much knowledge during our weekly telephone conference discussions. Thank you for contributing to this! I will surely miss the voice saying "you are the first participant in the meeting".

Elin Gursky - Thank you for valuable advice and critique on drafts of texts related to my research.

Benjamin Cowling - Your invaluable advice helped to improve the research in this thesis. Thank you! 
Lars Valter - Thank you for providing me with data; without these data this thesis would not be in its current form.

Kajsa Bendtsen - Your patience and support throughout my studies is greatly appreciated. Big thanks!

Camilla Rosén - Your help with the administration-related matters is highly valued. Thank you!

Lorna O'Brien - Thank you for invaluable assistance in correcting the English in this thesis and related texts.

Last but not least, my family:

My parents Fatima and Enes - you were always there for me and you gave me all I ever needed - you gave me love. Thank you for everything and please forgive me if I have caused you any pain! I love you from the bottom of my heart!

My dear wife Amila - you were the puzzle missing in my life. You have supported me since I met you. Thank you my love, I love you to the heaven and back!

My sister Ilana - you are an inspiration and it is and always will be a pleasure competing with you. You have a special place of love in my heart! Thank you for being you!

My lovely nephews Amin and Adnan, being with you and watching you grow up softens my heart and gives me tremendous joy. May God guide you and protect you from all evil!

Also thanks to my grandparents - living as well as deceased, my uncles living and deceased - and your families for all love, support and times of joy you have given me from the day I was born until today. 


\section{REFERENCES}

Althouse BM, Scarpino SV, Meyers LA, Ayers JW, Bargsten M, Baumbach $\mathrm{J}$, et al. Enhancing disease surveillance with novel data streams: challenges and opportunities. EPJ Data Sci 2015;4. pii:17.

Altman DG, Royston P. What do we mean by validating a prognostic model? Stat Med 2000;19:453-473.

Andersson E, Bock D, Frisén M. Modeling influenza incidence for the purpose of on-line monitoring. Stat Methods Med Res 2008;17:421-438.

Biggerstaff M, Alper D, Dredze M, Fox S, Fung IC, Hickmann KS, et al.; Influenza Forecasting Contest Working Group. Results from the centers for disease control and prevention's predict the 2013-2014 Influenza Season Challenge. BMC Infect Dis 2016;16:357.

Binder S, Levitt AM, Sacks JJ, Hughes JM. Emerging infectious diseases: public health issues for the 21st century. Science 1999;284(5418):1311-1313.

Bližňák V, Sokol Z, Zacharov P. Nowcasting of deep convective clouds and heavy precipitation: comparison study between NWP model simulation and extrapolation. Atmos Res 2017;184:24-34.

Bock D, Andersson E, Frisén M. Statistical surveillance of epidemics: peak detection of influenza in Sweden. Biom J 2008;50(1):71-85.

Boehm BW. Software engineering economics. New York: Prentice-Hall; 1981.

Bossuyt PM, Reitsma JB, Linnet K, Moons KG. Beyond diagnostic accuracy: the clinical utility of diagnostic tests. Clin Chem 2012;58(12):16361643.

Boyle JR, Sparks RS, Keijzers GB, Crilly JL, Lind JF, Ryan LM. Prediction and surveillance of influenza epidemics. Med J Aust 2011;194:S28-33.

Bregman DJ, Langmuir AD. Farr's law applied to AIDS projections. JAMA 1990;263(11):1522-1525.

Brillman JC, Burr T, Forslund D, Joyce E, Picard R, Umland E. Modeling emergency department visit patterns for infectious disease complaints: results and application to disease surveillance. BMC Med Inform Decis Mak 2005;5(4):1-14.

Browning KA, Collier CG. Nowcasting of precipitation systems. Rev Geophys 1989;27(3):345-370. 
Brownlee J. On the curve of the epidemic. Br Med J 1915;1(2836):799-800.

Buckeridge DL. Outbreak detection through automated surveillance: a review of the determinants of detection. J Biomed Inform 2007;40:370-379.

Buckeridge DL, Burkom H, Campbell M, Hogan WR, Moore AW. Algorithms for rapid outbreak detection: a research synthesis. J Biomed Inform 2005b;38(2):99-113.

Buckeridge DL, Okhmatovskaia A, Tu S, O'Connor M, Nyulas C, Musen MA. Predicting outbreak detection in public health surveillance: quantitative analysis to enable evidence-based method selection. AMIA Апnи Symp Proc 2008;76-80.

Buckeridge DL, Switzer P, Owens D, Siegrist D, Pavlin J, Musen M. An evaluation model for syndromic surveillance: assessing the performance of a temporal algorithm. MMWR 2005a;54:109-115.

Buehler JW, Whitney EA, Smith D, Prietula MJ, Stanton SH, Isakov AP. Situational uses of syndromic surveillance. Biosecur Bioterror 2009;7:165-177.

Burkom HS. Development, adaptation, and assessment of alerting algorithms for biosurveillance. Johns Hopkins APL Tech Dig 2003;24(4):335-342.

Burkom HS, Murphy SP, Shmueli G. Automated time series forecasting for biosurveillance. Stat Med 2007;26:4202-4218.

Butler D. When Google got flu wrong. Nature 2013;494(7436):155-156.

Castillo-Salgado C. Trends and directions of global public health surveillance. Epidemiol Rev 2010;32(1):93-109.

Centers for Disease Control and Prevention (CDC). Announcement of requirements and registration for the Predict the Influenza Season Challenge. Office of the Federal Register, National Archives and Records Administration. CDC 2013 URL:https://www.gpo.gov/fdsys/granule/FR-201311-25/2013-28198. Accessed: 21 November 2016. (Archived by WebCite at http://www.webcitation.org/6mBDujTFF).

Chretien JP, George D, Shaman J, Chitale RA, McKenzie FE. Influenza forecasting in human populations: a scoping review. PLoS One 2014;9(4):e94130.

Closas P, Coma E, Méndez L. Sequential detection of influenza epidemics by the Kolmogorov-Smirnov test. BMC Med Inform Decis Mak 2012;12:112. 
Cohen J. Statistical power analysis for the behavioral sciences (2nd edn). Mahwah, NJ: Lawrence Erlbaum; 1988.

Collins GS, Reitsma JB, Altman DG, Moons KG; members of TRIPOD group. Transparent reporting of a multivariable prediction model for individual prognosis or diagnosis (TRIPOD): the TRIPOD statement. BMJ 2015;350:g7594.

Corley CD, Pullum LL, Hartley DM, Benedum C, Noonan C, Rabinowitz PM, et al. Disease prediction models and operational readiness. PLoS One 2014;9:e91989.

Cowling BJ, Wong IO, Ho LM, Riley S, Leung GM. Methods for monitoring influenza surveillance data. Int J Epidemiol 2006;35(5):1314-1321.

Derose SF, Schuster MA, Fielding JE, Asch SM. Public health quality measurement: concepts and challenges. Annu Rev Public Health 2002;23:1-21.

Dixon M, Wiener G. TITAN: thunderstorm identification, tracking, analysis, and nowcasting - a radar-based methodology. I Atmos Oceanic Technol 1993;10(6):785-797.

Dohoo I, Martin W, Stryhn H. Veterinary epidemiologic research (2nd edn). Charlottetown, Canada: Atlantic Veterinary College; 2010.

Donker T, van Boven M, van Ballegooijen WM, Van't Klooster TM, Wielders CC, Wallinga J. Nowcasting pandemic influenza A/H1N1 2009 hospitalizations in the Netherlands. Eur J Epidemiol 2011;26(3):195-201.

Dórea FC, Lindberg A, McEwen BJ, Revie CW, Sanchez J. Syndromic surveillance using laboratory test requests: a practical guide informed by experience with two systems. Prev Vet Med 2014;116(3):313-324.

Dórea FC, McEwen BJ, McNab WB, Revie CW, Sanchez J. Syndromic surveillance using veterinary laboratory data: data pre-processing and algorithm performance evaluation. J R Soc Interface 2013a;10(83):20130114.

Dórea FC, McEwen BJ, McNa WB, Sanchez J, Revie CW. Syndromic surveillance using veterinary laboratory data: algorithm combination and customization of alerts. PLoS One 2013b;8(12):e82183.

Dugas AF, Hsieh YH, Levin SR, Pines JM, Mareiniss DP, Mohareb A, et al. Google Flu Trends: correlation with emergency department influenza rates and crowding metrics. Clin Infect Dis 2012;54(4):463-469.

Dugas AF, Jalalpour M, Gel Y, Levin S, Torcaso F, Igusa T, et al. Influenza forecasting with Google Flu Trends. PLoS One 2013;8:e56176. 
European Centre for Disease Prevention and Control (ECDC). Evidencebased methodologies for public health - how to assess the best available evidence when time is limited and there is lack of sound evidence. Sweden, Stockholm: ECDC 2011.

Friesema IH, Koppeschaar CE, Donker GA, Dijkstra F, van Noort SP, Smallenburg $R$, et al. Internet-based monitoring of influenza-like illness in the general population: experience of five influenza seasons in The Netherlands. Vaccine 2009;27(45):6353-6357.

Gerbier-Colomban S, Potinet-Pagliaroli V, Metzger MH. Can epidemic detection systems at the hospital level complement regional surveillance networks: case study with the influenza epidemic? BMC Infect Dis 2014;14:381.

Goel S, Hofman JM, Lahaie S, Pennock DM, Watts DJ. Predicting consumer behavior with Web search. Proc Natl Acad Sci $U S A$ 2010;107(41):17486-17490.

Golding BW. Nimrod. A system for generating automated very short range forecasts. Meteorol Appl 1998;5(1):1-16.

Greenhalgh T, Robert G, Macfarlane F, Bate P, Kyriakidou O, Peacock R. Storylines of research in diffusion of innovation: a meta-narrative approach to systematic review. Soc Sci Med 2005;61:417-430.

Gursky EA, Bice G. Assessing a decade of public health preparedness: progress on the precipice? Biosecur Bioterror 2012;10:55-65.

Hastie T, Tibshirani R, Friedman J. The elements of statistical learning: data mining, inference, and prediction (2nd edn). New York: Springer-Verlag; 2009.

Hii YL, Zhu H, Ng N, Ng LC, Rocklöv J. Forecast of dengue incidence using temperature and rainfall. PLoS Negl Trop Dis 2012;6:e1908.

Hiller KM, Stoneking L, Min A, Rhodes SM. Syndromic surveillance for influenza in the emergency department-A systematic review. PLoS One 2013;8(9):e73832.

Hosmer DW, Lemeshow S. Applied logistic regression (2nd edn). London: John Wiley; 2000, pp. 228-230.

Jiang X, Wallstrom G, Cooper GF, Wagner MM. Bayesian prediction of an epidemic curve. J Biomed Inform 2009;42:90-99.

Jones KE, Patel NG, Levy MA, Storeygard A, Balk D, Gittleman JL, et al. Global trends in emerging infectious diseases. Nature 2008;451(7181):990-993. 
Kang M, Zhong H, He J, Rutherford S, Yang F. Using Google Trends for influenza surveillance in South China. PLoS One 2013;8(1):e55205.

Kaufman Z, Wong WK, Peled-Leviatan T, Cohen E, Lavy C, Aharonowitz $G$, et al. Evaluation of a syndromic surveillance system using the WSARE algorithm for early detection of an unusual, localized summer outbreak of influenza B: implications for bioterrorism surveillance. Isr Med Assoc J 2007;9(1):3-7.

Keller M, Blench M, Tolentino H, Freifeld CC, Mandl KD, Mawudeku A, et al. Use of unstructured event-based reports for global infectious disease surveillance. Emerg Infect Dis 2009;15:689-695.

Kelly M, Morgan A, Ellis S, Younger T, Huntley J, Swann C. Evidence based public health: a review of the experience of The National Institute of Health and Clinical Excellence (NICE) of developing public health guidance in England. Soc Sci Med 2010;71:1056-1062.

Kim EK, Seok JH, Oh JS, Lee HW, Kim KH. Use of hangeul twitter to track and predict human influenza infection. PLoS One 2013;8(7):e69305.

Kirian ML, Weintraub JM. Prediction of gastrointestinal disease with over-the-counter diarrheal remedy sales records in the San Francisco Bay Area. BMC Med Inform Decis Mak 2010;10:39.

Klick B, Nishiura H, Leung GM, Cowling BJ. Optimal design of studies of influenza transmission in households II: comparison between cohort and case-ascertained studies. Epidemiol Infect 2014;142:744-752.

Laporte RE. How to improve monitoring and forecasting of disease patterns. BMJ 1993;307(6919):1573-1574.

Last JM (ed). A dictionary of epidemiology. Oxford: Oxford University Press; 2001.

Lazer D, Kennedy R, King G, Vespignani A. Big data. The parable of Google Flu: traps in big data analysis. Science 2014;343(6176):1203-1205.

Liu TY, Sanders JL, Tsui FC, Espino JU, Dato VM, Suyama J. Association of over-the-counter pharmaceutical sales with influenza-like-illnesses to patient volume in an urgent care setting. PLoS One 2013;8(3):e59273.

Lotze T, Murphy S, Shmueli G. Implementation and comparison of preprocessing methods for biosurveillance data. Adv Dis Surveill 2008;6(1):120. 
Lotze TH, Shmueli G, Yahav I, Kass-Hout T, Zhang X. Simulating and evaluating biosurveillance datasets. In: Kass-Hout $T$, Zhang $X$, eds. Biosurveillance: methods and case studies. New York: CRC Press; 2011, pp. 23-51.

Louz D, Bergmans HE, Loos BP, Hoeben RC. Emergence of viral diseases: mathematical modeling as a tool for infection control, policy and decision making. Crit Rev Microbiol 2010;36:195-211.

Ma T, Englund H, Bjelkmar P, Wallensten A, Hulth A. Syndromic surveillance of influenza activity in Sweden: an evaluation of three tools. Epidemiol Infect 2015;143(11):2390-2398.

Malik MT, Gumel A, Thompson LH, Strome T, Mahmud SM. "Google flu trends" and emergency department triage data predicted the 2009 pandemic H1N1 waves in Manitoba. Can J Public Health 2011;102(4):294-297.

Martínez-Beneito MA, Conesa D, López-Quílez A, López-Maside A. Bayesian Markov switching models for the early detection of influenza epidemics. Stat Med 2008;27:4455-4468.

Mays N, Pope C, Popay J. Systematically reviewing qualitative and quantitative evidence to inform management and policy-making in the health field. J Health Serv Res Policy 2005;10(Suppl 1):6-20.

Merler S, Ajelli M, Pugliese A, Ferguson NM. Determinants of the spatiotemporal dynamics of the $2009 \mathrm{H} 1 \mathrm{N1}$ pandemic in Europe: implications for real-time modelling. PLoS Comput Biol 2011;7(9):e1002205.

Milinovich GJ, Williams GM, Clements AC, Hu W. Internet-based surveillance systems for monitoring emerging infectious diseases. Lancet Infect Dis 2014;14(2):160-168.

Molinari N-AM, Ortega-Sanchez IR, Messonnier ML, Thompson WW, Wortley PM, Weintraub E, et al. The annual impact of seasonal influenza in the US: measuring disease burden and costs. Vaccine 2007;5086-5096.

Moniz L, Buczak AL, Baugher B, Guven E, Chretien JP. Predicting influenza with dynamical methods. BMC Med Inform Decis Mak 2016;16(1):134.

Montgomery DC. Introduction to statistical quality control. Hoboken, NJ: John Wiley; 2005.

Morens DM, Folkers GK, Fauci AS. The challenge of emerging and reemerging infectious diseases. Nature 2004;430(6996):242-249. 
Moss R, Zarebski A, Dawson P, McCaw JM. Retrospective forecasting of the 2010-2014 Melbourne influenza seasons using multiple surveillance systems. Epidemiol Infect 2017;145(1):156-169.

Nagel AC, Tsou MH, Spitzberg BH, An L, Gawron JM, Gupta DK, et al. The complex relationship of realspace events and messages in cyberspace: case study of influenza and pertussis using tweets. J Med Internet Res 2013;15(10):e237.

Neuberger A, Paul M, Nizar A, Raoult D. Modelling in infectious diseases: between haphazard and hazard. Clin Microbiol Infect 2013;19:993-998.

Nishiura H. Lessons from previous predictions of HIV/AIDS in the United States and Japan: epidemiologic models and policy formulation. Epidemiol Perspect Innov 2007;4:3.

Nsoesie EO, Brownstein JS, Ramakrishnan N, Marathe MV. A systematic review of studies on forecasting the dynamics of influenza outbreaks. Influenza Other Respir Viruses 2014;8(3):309-316.

Ohkusa Y, Sugawara T, Taniguchi K, Okabe N. Real-time estimation and prediction for pandemic $\mathbf{A} / \mathbf{H} 1 \mathbf{N 1 ( 2 0 0 9 )}$ in Japan. I Infect Chemother 2011;17:468-472.

Ortiz JR, Zhou H, Shay DK, Neuzil KM, Fowlkes AL, Goss CH. Monitoring influenza activity in the United States: a comparison of traditional surveillance systems with Google Flu Trends. PLoS One 2011;6(4):e18687.

Paterson BJ, Durrheim DN. The remarkable adaptability of syndromic surveillance to meet public health needs. J Epidemiol Glob Health 2013;3:41-47.

Pollett S, Boscardin WJ, Azziz-Baumgartner E, Tinoco YO, Soto G, Romero $\mathrm{C}$, et al. Evaluating Google flu trends in Latin America: important lessons for the next phase of digital disease detection. Clin Infect Dis 2017;64(1):34-41.

Reich NG, Cummings DA, Lauer SA, Zorn M, Robinson C, Nyquist AC, et al. Triggering Interventions for Influenza: the ALERT Algorithm. Clin Infect Dis 2015;60:499-504.

Riley RD, Ensor J, Snell KI, Debray TP, Altman DG, Moons KG, et al. External validation of clinical prediction models using big datasets from ehealth records or IPD meta-analysis: opportunities and challenges. $B M J$ 2016;353:i3140. 
Rolland-Harris E, Mangtani P, Moore KM. Who uses telehealth? Setting a usage baseline for the early identification of pandemic influenza activity. Telemed J E Health 2012;18(2):153-157.

Santos-Preciado J, Franco-Paredes C, Hernandez-Flores I, Tellez I, Del Rio $\mathrm{C}$, Tapia-Conyer $\mathrm{R}$. What have we learned from the novel influenza A (H1N1) pandemic in $\mathbf{2 0 0 9}$ for strengthening pandemic influenza preparedness? Arch Med Res 2009;40:673-676.

Schanzer D, Vachon J, Pelletier L. Age-specific differences in influenza A epidemic curves: do children drive the spread of influenza epidemics? Am J Epidemiol 2011;174(1):109-117.

Schiöler L. Modelling the spatial patterns of influenza incidence in Sweden. Gothenburg: Statistical Research Unit, Department of Economics, University of Gothenburg, Sweden 2010. Report no. 2010:1.

Serfling RE. Methods for current statistical analysis of excess pneumonia-influenza deaths. Public Health Rep 1963;78:494-506.

Shaman J, Kandula S. Improved discrimination of influenza forecast accuracy using consecutive predictions. PLoS Curr 2015;7.

Shaman J, Karspeck A. Forecasting seasonal outbreaks of influenza. Proc Natl Acad Sci U S A 2012;109:20425-20430.

Shaman J, Karspeck A, Yang W, Tamerius J, Lipsitch M. Real-time influenza forecasts during the 2012-2013 season. Nat Commun 2013;4:2837.

Sharpe JD, Hopkins RS, Cook RL, Striley CW. Evaluating Google, Twitter, and Wikipedia as tools for influenza surveillance using Bayesian change point analysis: a comparative analysis. JMIR Public Health Surveill 2016;2(2):e161.

Singh BK, Savill NJ, Ferguson NM, Robertson C, Woolhouse ME. Rapid detection of pandemic influenza in the presence of seasonal influenza. $B M C$ Public Health 2010;10:726.

Socan M, Erculj V, Lajovic J. Early detection of influenza like illness through medication sales. Cent Eur J Public Health 2012;20(2):156-162.

Spreco A, Timpka T. Algorithms for detecting and predicting influenza outbreaks: meta-narrative review of prospective evaluations. BMJ Open 2016;6:e010683. 
Stroup DF, Williamson GD, Herndon JL, Karon JM. Detection of aberrations in the occurrence of notifiable diseases surveillance data. Stat Med 1989;8:323-329; discussion 331-332.

Sveriges Kommuner och Landsting (SKL). Ingen på sjukhus $i$ onödan. Stockholm: Sveriges Kommuner och Landsting, 2016 [in Swedish].

Szucs T. The socio-economic burden of influenza. J Antimicrob Chemother 1999;44(Suppl B):11-15.

Thokala P, Devlin N, Marsh K, Baltussen R, Boysen M, Kalo Z, et al. Multiple criteria decision analysis for health care decision making--an introduction: Report 1 of the ISPOR MCDA Emerging Good Practices Task Force. Value Health 2016;19(1):1-13.

Thorrington D, Balasegaram S, Cleary P, Hay C, Eames K. Social and Economic Impacts of School Influenza Outbreaks in England: Survey of Caregivers. J Sch Health 2017;87(3):209-216.

Timpka T, Eriksson H, Gursky EA, Strömgren M, Holm E, Ekberg J, et al. Requirements and design of the PROSPER protocol for implementation of information infrastructures supporting pandemic response: a Nominal Group study. PLoS One 2011;6(3):e17941.

Timpka T, Eriksson O, Spreco A, Gursky EA, Strömgren M, Holm E, et al. Age as a determinant for dissemination of seasonal and pandemic influenza: an open cohort study of influenza outbreaks in Östergötland County, Sweden. PLoS One 2012;7(2):e31746.

Timpka T, Spreco A, Dahlström Ö, Eriksson O, Gursky EA, Ekberg J, et al. Performance of eHealth data sources in local influenza surveillance: a 5-year open cohort study. J Med Internet Res 2014b;16(4):e116.

Timpka T, Spreco A, Eriksson O, Dahlström Ö, Gursky EA, Strömgren M, et al. Predictive performance of telenursing complaints in influenza surveillance: a prospective cohort study in Sweden. Euro Surveill 2014a;19:46.

Tokars JI, Burkom H, Xing J, English R, Bloom S, Cox K, et al. Enhancing time-series detection algorithms for automated biosurveillance. Emerg Infect Dis 2009;15(4):533-539.

Unkel S, Farrington CP, Garthwaite PH, Robertson C, Andrews N. Statistical methods for the prospective detection of infectious disease outbreaks: a review. J Roy Stat Soc A Sta 2012;175(Part1):49-82.

Vega T, Lozano JE, Meerhoff T, Snacken R, Beauté J, Jorgensen P, et al. Influenza surveillance in Europe: comparing intensity levels calculated 
using the moving epidemic method. Influenza Other Respir Viruses 2015;9(5):234-246.

Vega T, Lozano JE, Meerhoff T, Snacken R, Mott J, Ortiz de Lejarazu, et al. Influenza surveillance in Europe: establishing epidemic thresholds by the moving epidemic method. Influenza Other Respir Viruses 2013;7:546-558.

Viboud C, Boelle PY, Carrat F, Valleron AJ, Flahault A. Prediction of the spread of influenza epidemics by the method of analogues. Am J Epidemiol 2003;158:996-1006.

Wahlberg AC, Wredling R. Telephone nursing: calls and caller satisfaction. Int J Nurs Pract 1999;5(3):164-170.

Watkins RE, Eagleson S, Veenendaal B, Wright G, Plant AJ. Applying cusum-based methods for the detection of outbreaks of Ross River virus disease in Western Australia. BMC Med Inform Decis Mak 2008;8:37.

White LF, Wallinga J, Finelli L, Reed C, Riley S, Lipsitch M, et al. Estimation of the reproductive number and the serial interval in early phase of the 2009 influenza A/H1N1 pandemic in the USA. Influenza Other Respir Viruses 2009;3:267-276.

Wilson N, Mason K, Tobias M, Peacey M, Huang QS, Baker M. Interpreting Google flu trends data for pandemic H1N1 influenza: the New Zealand experience. Euro Surveill 2009;14(44):1-3.

Wong G, Greenhalgh T, Westhorp G, Buckingham J, Pawson R. RAMESES publication standards: meta-narrative reviews. $B M C \mathrm{Med}$ 2013;11:20.

World Health Organization (WHO). WHO 2016 URL:http://www.who.int/mediacentre/factsheets/fs211/en/. Accessed: 13 January 2017. (Archived by WebCite at http://www.webcitation.org/6nTrzDSkV).

World Health Organization (WHO). Media centre - Influenza A(H1N1) Statement by WHO Director-General, Dr Margaret Chan. WHO 2009 URL:http://www.who.int/mediacentre/news/statements/2009/h1n1_20090429/ en/. Accessed: 15 January 2017. (Archived by WebCite at http://www.webcitation.org/6nXGUhmSr).

World Meteorological Organization (WMO). Nowcasting. WMO 2017 URL:http://www.wmo.int/pages/prog/amp/pwsp/Nowcasting.htm. Accessed: 6 April 2017. (Archived by WebCite at http://www.webcitation.org/6pWEcMZde). 
Yang W, Karspeck A, Shaman J. Comparison of filtering methods for the modeling and retrospective forecasting of influenza epidemics. PLoS Comput Biol 2014;10(4):e1003583.

Yang W, Lipsitch M, Shaman J. Inference of seasonal and pandemic influenza transmission dynamics. Proc Natl Acad Sci U S A 2015;112(9):27232728.

Yom-Tov E, Borsa D, Cox IJ, McKendry RA. Detecting disease outbreaks in mass gatherings using Internet data. J Med Internet Res 2014;16(6):e154.

Yuan Q, Nsoesie EO, Lv B, Peng G, Chunara R, Brownstein JS. Monitoring influenza epidemics in China with search query from Baidu. PLoS One 2013;8:e64323. 


\section{Papers}

The articles associated with this thesis have been removed for copyright reasons. For more details about these see:

http://urn.kb.se/resolve?urn=urn:nbn:se:liu:diva-136553 